\title{
Importance of Mullins effect in commercial silicone elastomer formulations for soft robotics
}

\author{
Krpovic, Sara; DamJohansen, Kim; Skov, Anne Ladegaard
}

Published in:

Journal of Applied Polymer Science

Link to article, DOI:

10.1002/app.50380

Publication date:

2021

Document Version

Peer reviewed version

Link back to DTU Orbit

Citation (APA):

Krpovic, S., DamJohansen, K., \& Skov, A. L. (2021). Importance of Mullins effect in commercial silicone elastomer formulations for soft robotics. Journal of Applied Polymer Science, 138(19), [50380]. https://doi.org/10.1002/app.50380

\section{General rights}

Copyright and moral rights for the publications made accessible in the public portal are retained by the authors and/or other copyright owners and it is a condition of accessing publications that users recognise and abide by the legal requirements associated with these rights.

- Users may download and print one copy of any publication from the public portal for the purpose of private study or research.

- You may not further distribute the material or use it for any profit-making activity or commercial gain

- You may freely distribute the URL identifying the publication in the public portal

If you believe that this document breaches copyright please contact us providing details, and we will remove access to the work immediately and investigate your claim 


\section{WILEY-VCH}

\section{Importance of Mullins effect in commercial silicone elastomer formulations for soft robotics}

\section{Sara Krpovic, Kim Dam-Johansen and Anne Ladegaard Skov*}

Sara Krpovic, Prof. Kim Dam-Johansen

Technical University of Denmark, Department of Chemical and Biochemical Engineering, The Hempel Foundation Coatings Science and Technology Centre

Kgs. Lyngby 2800, Denmark.

E-mail: sarakr@kt.dtu.dk,kdj@kt.dtu.dk

Anne Ladegaard Skov

Technical University of Denmark, Department of Chemical and Biochemical Engineering, Danish Polymer Centre, Kgs. Lyngby 2800, Denmark.

E-mail: al@kt.dtu.dk

Keywords: Mullins effect, silicone elastomers, cyclic uniaxial tension tests, soft robotics

Silicone elastomers are used in a wide range of applications, including artificial muscles, biomedical devices, and soft robotics, for which chemical, thermal, and mechanical stability are important requirements that these elastomers must fulfill. However, to ensure that silicone elastomers' properties and performance remain constant under long term deployment, it is necessary to examine and account for the Mullins effect, which has the potential to significantly alter certain elastomer properties of interest. In this article, the mechanical properties of soft and hard commercial silicone elastomers and two blends of commercial silicone elastomers are investigated - specifically their softening behavior due to the Mullins effect. Ultimate stresses, ultimate strains, and Young's moduli are obtained from uniaxial tensile tests. Results show that the point of softening greatly depends on both the elastomer type and its strain history. Furthermore, a significant permanent set is observed in the softest commercial formulations.

\section{Introduction}

Silicone elastomers are becoming increasingly important in modern technology as a result of their multiple applications in soft robotics. Silicone elastomers' exceptional versatility for soft robotics arises from their inherent softness, as well as their thermal, and chemical stability. ${ }^{[1,2]}$ The similarity of their mechanical properties to those of human muscles facilitates biomimicry. ${ }^{[3,4]}$ The era of the elastomers is coming, but the underlying theory is easily 


\section{WILEY-VCH}

forgotten. Simplistic models were initially developed in the 1940 's. ${ }^{[5-9]}$ They were followed by more complicated but more realistic rubber elasticity theories. Recent advances into modelling of elastic phenomena have become increasingly complex and thus more difficult to understand. What is causing the mechanical properties of (silicone) elastomers to become so complicated to fully describe? First of all, most commercial silicone elastomers consist of a covalently crosslinked network of polymer chains that are: 1) fully elastic - defined as a chain connected to an active crosslinker at both ends; 2) dangling - defined as a chain connected to an active crosslinker at one end only; or 3) soluble - defined as a chain being not connected to an active crosslinker, also denoted the sol fraction. ${ }^{[10,11]}$ An active crosslinker is defined as a crosslinker that is connected to the infinite network by more than 2 covalent bonds. The structure of this covalent network is challenging to describe theoretically due to complex statistics, not to mention imperfections in the reactants that further complicate the determinations. ${ }^{[12]}$ Furthermore, all three types of network constituents have different dynamics, which additionally depend on their structure and molecular weight. Hierarchical relaxation therefore prevails; however, due to the vast number of structures with different relaxation times, the overall relaxation spectrum will be extremely complicated. Elastic polymer chains can be modelled as elastic springs (Hookean behavior) if they are relatively short (with a molecular weight below the entanglement threshold, occurring at approximately $2 \mathrm{M}_{\mathrm{e}}=24.000 \mathrm{~g} / \mathrm{mol}$ for polydimethylsiloxane (PDMS) $)^{[13]}$. However, when these chains become longer, more complex phenomena take place due to entanglements and the complicated relaxation processes related to them. ${ }^{[14-16]}$ Dangling chains and branched substructures have very long time scales of relaxation and therefore act as elastic strands on short time-scales and as plasticizers on longer time-scales. ${ }^{[17]}$ The sol fraction acts as a plasticizer at most relevant time-scales. In addition to the complex behavior of the elastomer strands themselves, there are also almost always fillers of varying types present. For silicone elastomers, the most dominating by mass, and effect, are silica fillers, ${ }^{[18]}$ which can be replaced by resins if optical transparency or low viscosity are 


\section{WILEY-VCH}

needed for a given application. However, when resins are present, additional covalent bonds are introduced into the network (as compared to non-covalent interactions with, e.g. silica) and the polymer network formed has a higher crosslinking density than that of networks with only silica fillers present in the same concentration. Fillers are most commonly added to silicone elastomers in order to produce a required mechanical strength especially tear strength, as well as to lower the price of the elastomer. A common rule of thumb is that the percolation threshold for silica in silicone is about $5 \mathrm{phr}$ (parts per hundred rubber). ${ }^{[19]}$ Solid fillers in the silicone matrix are rarely found as single, individual particles. Rather, the particles usually form clusters, known as aggregates, which form agglomerates via further joining. ${ }^{[20]}$ In other words, when silica is present in silicone elastomers, there are also particle-particle and particle-polymer interactions to account for in addition to the covalent interactions. All these different interactions, together with the nano- and micro-scale damages within the elastomer, reduce the reversibility of the elastomer dimensions after subjection to a cyclic strain deformation: this is called the Mullins effect. ${ }^{[8,21-23]}$ In rheological terms, it means that the elastomer has a stress memory function, such that the stress at a given strain will depend on the strain history of the sample. Even though the Mullins effect has been observed in unfilled elastomer rubbers, this strain-induced phenomenon is more pronounced in filler-reinforced elastomers. ${ }^{[24]}$

Research over the past several decades has given different explanations for the physical mechanisms behind the Mullins effect, including polymer-filler chain breakage ${ }^{[25-27]}$, sliding of molecular chains ${ }^{[28]}$, rupture of filler clusters ${ }^{[29]}$, and chain disentanglement ${ }^{[30]}$. However, a comprehensive explanation of this mechanism has yet to be articulated, though the effect most likely depends on a wide range of different parameters such as: type of rubber and additives, presence of resins, filler type and aspect ratio, filler modification, and strain rate. ${ }^{[31]}$ Without a comprehensive theory to rely on, then how and where, do we observe the Mullins effect in practical terms? A common way to make thin elastomers is to coat the liquid silicone elastomer mixture onto a substrate - e.g., a plastic film or glass plate - and then cure the elastomer on the 


\section{WILEY-VCH}

substrate. The elastomer is then released from the substrate after curing, but, since the adhesion forces are relatively large compared to the elastic forces of the thin silicone elastomer, it undergoes stretching that is often beyond the elastic regime of its particle-particle interactions. The mechanical properties of the elastomer may already have experienced the Mullins effect by this stretch, and in this way its material properties become sensitive to processing conditions. Elastomers are typically observed to exhibit their highest Young's modulus in the first deformation cycle; when reporting data or designing products, one must therefore be aware that the first cycle at, e.g., constant stress, will give lower strain response than the subsequent steadystate cycles. ${ }^{[19]}$

Reversible stretchability and flexibility render silicone elastomers suitable materials for soft transducers $^{[32-34]}$, soft robots ${ }^{[35]}$, medical devices ${ }^{[32,36]}$, and many other futuristic applications. Silicone elastomers used for different applications are expected to maintain reliable properties and performance under long-term employment. Accounting for the Mullins effect in silicone formulations offers a simple means of avoiding experimental discrepancies and product unreliability. The Mullins effect has been widely characterized and modeled in natural rubbers with and without fillers dispersed..$^{[7,23,28,31,33,35,37-40]}$ Amongst silicone elastomers, Sylgard formulations are by far the most investigated ones, and Sylgard 184 is often considered as a benchmark for commercial silicone elastomers. ${ }^{[33,35,39]}$ However, the Ecoflex formulations with Shore hardness values ranging from 00-10 to 00-50 have lately gained significant attention in different applications due to their increased softness and stretchability compared to the more rigid Sylgard 184. ${ }^{[34,35,38,41,42]}$ While investigations of Sylgard formulations are extensive, investigations of Ecoflex are still quite limited, and a thorough, consistent characterization of the Ecoflex remains lacking. In this work, four commercial silicone formulations (Sylgard 184, Sylgard 186, Ecoflex 00-30 and Ecoflex 00-50), two combinations of commercial silicone formulations (Sylgard 184 and Ecoflex 00-30, Sylgard 184 and Ecoflex 00-50) and two silicone formulations mixed at different stoichiometric ratios (Sylgard 184 and Sylgard 186) were made 


\section{WILEY-VCH}

and tested in order to illustrate commonly encountered strain-induced phenomena. Moreover, correlations between softening effect and different silicone elastomer formulations were made using the strain energy function.

\section{Materials and sample preparation}

Four different two-part (A and B) commercial Pt-curing silicone elastomers are used. Sylgard 184 and Sylgard 186 silicone elastomers are obtained from Dow Corning, Germany, while Ecoflex 00-30 and Ecoflex 00-50 elastomers, are obtained from FormX, Netherlands. The term "blend" will be used throughout the article to refer to the formulations obtained by combining two different commercial silicone formulations. Sample compositions are shown in Table 1. Parts A and B are weighed according to the mixing ratio and mixed in a SpeedMixer ${ }^{\mathrm{TM}}$ DAC $150 \mathrm{FVZ}-\mathrm{K}$ for 2 minutes at $2000 \mathrm{rpm}$. Since resins and inorganic fillers (most commonly silica fillers) are already present in silicone elastomer formulations, the elastomers are prepared as received from the supplier, without introduction of additional fillers.

In the case of blends, two different formulations are blended in the ratios specified in Table 1 for another 2 minutes at $2000 \mathrm{rpm}$, after which the blends are used to prepare samples. The manufacturer's recommended mixing ratio for Sylgard formulations is 10:1 (part A:part B), while for Ecoflex formulations the ratio is 1:1 (part A:part B). To make films, mixtures are then blade casted on acetate/polyester support film (purchased from Pütz GmbH + Co. Folien KG, Germany). A blade gap of $400 \mu \mathrm{m}$ is used in order to obtain an approximately $300 \mu \mathrm{m}$ thick film after curing. Samples are cured at $80^{\circ} \mathrm{C}$ for 2 hours. After curing, all films have a thickness of $300 \mu \mathrm{m} \pm 25 \mu \mathrm{m}$. The precise sample thickness is determined using optical microscopy. Since no solvents are used, a direct post-curing process ${ }^{[43]}$ is rendered unnecessary and samples are expected to be fully cured by the procedure described above.

\section{Measurements}

\subsection{Fourier Transform Infrared Spectroscopy Analysis}




\section{WILEY-VCH}

Fourier transform infrared (FTIR) spectroscopy is carried out using a Nicolet iS50 FTIR spectrometer (Thermo Fisher Scientific, USA). Wavenumbers between 400 and $4000 \mathrm{~cm}-1$ are recorded with 64 interferograms for each sample formulation as well as their respective A and B parts, in transmission mode.

\subsection{Extraction measurements}

The gel fractions are determined using gel extraction, in which small pieces of the sample film ( 200 mg) are immersed in chloroform $(\sim 10 \mathrm{~mL})$ for $48 \mathrm{~h}$ at room temperature (approximately $23^{\circ} \mathrm{C}$ ). The solvent is replaced after $24 \mathrm{~h}$; after $48 \mathrm{~h}$, the solvent is poured out and the films are washed twice with fresh solvent. The samples are then dried for $48 \mathrm{~h}$ at room temperature under ambient pressure, until constant weight is obtained. Gel fractions are calculated as $\mathrm{w}_{\text {gel }}=\mathrm{m}_{\mathrm{e}} / \mathrm{m}_{0}$, where $m_{e}$ is the weight after extraction and drying, and $m_{0}$ is the initial weight of the sample. ${ }^{[44]}$

\subsection{Thermogravimetry analysis}

Thermogravimetry analysis (TGA) of the sample films is performed on a thermogravimetric analyzer Discovery series (TA instruments). Samples $(\sim 10 \mathrm{mg})$ are heated from room temperature to $900^{\circ} \mathrm{C}$ in a platinum pan under a nitrogen flow $(25 \mathrm{~mL}$ min-1) at a heating rate of $10^{\circ} \mathrm{C}$ min-1. Measurements are repeated 3 times.

\subsection{Nuclear magnetic resonance spectroscopy}

Nuclear magnetic resonance (NMR) spectroscopy is performed on a Bruker $300 \mathrm{MHz}$ spectrometer. A and B parts of Ecoflex 00-30 and Ecoflex 00-50 formulations (approximately $50 \mathrm{mg})$ are dissolved in $0.7 \mathrm{~mL}$ of chloroform-d $(\mathrm{CDCl} 3)$. Data are analyzed using TopSpin version $3.5 \mathrm{pl} 7$ from Bruker.

\subsection{Size exclusion chromatography}

Size exclusion chromatography (SEC) is performed on a chromatographic system consisting of a PL-ELS 2100 module connected to a Viscotek TriSEC model 302 triple detector array (RI, light scattering, viscometer). The PL Guard and two PL gel mixed D columns from Polymer Laboratories are connected in series, providing resolution up to 300,000 Daltons. The system 


\section{WILEY-VCH}

is calibrated using linear polydimethylsiloxane (PDMS) standards acquired from PSS (Mainz, Germany). SEC measurements of A and B parts of soft and hard formulations are performed. Both A and B parts of soft formulations are first dissolved in toluene and filtered through Celite (Celite ${ }^{\circledR} 545$, Merck KGaA) and filter paper (VWR), retaining particles 5-13 $\mu$ m in size. A and B parts of soft and hard formulations (approximately $2.5 \mathrm{mg}$ ) are dissolved in $1 \mathrm{~mL}$ of toluene and again filtered through a filter syringe with $0.45 \mu \mathrm{m}$ pore size. Samples are run at a room temperature $\left(23^{\circ} \mathrm{C}\right)$ in tetrahydrofuran $(\mathrm{THF})$ as mobile phase, with a flow rate of $1 \mathrm{~mL} \mathrm{~min}{ }^{-1}$. Molar mass characteristics are calculated using OmniSEC software.

\subsection{Sample thickness measurements}

Silicone film sheets of around $300 \mathrm{~cm}^{2}$ in area are prepared for each composition. In order to determine the film thickness small sample pieces (around $2 \times 10 \mathrm{~mm}$ ) are cut out from three different locations on the sample sheet and the thickness of cross-sections of these pieces is evaluated using optical microscopy. Three thickness measurements are performed for each piece (9 values in total) in order to calculate the average thickness and standard deviation of the silicone film sheets.

\subsection{Mechanical measurements}

Stress-strain measurements and cyclic uniaxial tension tests are performed on an Instron 3345 tensile tester equipped with a $500 \mathrm{~N}$ load cell. Stress-strain measurements are performed in order to determine ultimate mechanical properties, which will present limits for the subsequent cyclic uniaxial tension testing. For both tests with Instron, dog bone-shaped (Figure 1S. in SI) samples $115 \mathrm{~mm}$ in length, $25 \mathrm{~mm}$ and $6 \mathrm{~mm}$ in width (wide and narrow parts, respectively) and $300 \mu \mathrm{m} \pm 20 \mu \mathrm{m}$ in thickness are used. When samples are clamped onto the test fixtures, the measuring area is $75 \mathrm{~mm}$ in length, $6 \mathrm{~mm}$ in width, and $300 \mu \mathrm{m}$ in thickness. For stressstrain measurements, samples are stretched with a $2 \mathrm{~mm} / \mathrm{s}$ strain rate until broken, and measurements are repeated 4 times. Strain rate is also $2 \mathrm{~mm} / \mathrm{s}$ for cyclic uniaxial tests, but the measurements are performed 3 times for each composition. All measurements are performed at 


\section{WILEY-VCH}

room temperature (approximately $23^{\circ} \mathrm{C}$ ). Bluehill Universal software is used to calculate the ultimate mechanical properties of different formulations: Young's modulus, Y (as a tangent at $10 \%$ strain), ultimate elongation at break, $\varepsilon_{\mathrm{T}}$, and ultimate tensile strength $\sigma_{\mathrm{T}}$. All reported stresses are engineering stresses, i.e. forces per initial cross-sectional area of the samples. Two different types of cyclic tests are performed. One cycle is defined as the stretching of the sample to a certain strain value (loading path), after which the stretching force is removed and the sample is relaxed to a state of zero stress (unloading path). Three cycles are performed for each strain in all measurements.

In the first experimental test, samples are first stretched to $20 \%$ strain and then relaxed to a state of zero stress. Strain is further increased every three cycles until it reaches a value close to the value of strain at break for each composition ("strain rise path"), see Figure 1a). After stretching the sample to maximum strain, strain is reduced following the "strain drop path". The term " 1 st test" will be added to the sample name and used throughout the rest of this article to refer to the type of experimental test described above.

In the second experimental test, the samples are first stretched to a strain close to the strain at break for each composition. Strain is then incrementally reduced every three cycles until $20 \%$ strain is reached ("strain drop path"), see Figure 1b). After stretching the sample to $20 \%$ strain, strain is again increased following the "strain rise path". The term " 2 nd test" will be added to the sample name and used throughout the rest of the article to refer to the experimental test described above.

\section{Results and Discussion}

Below, the respective formulations will be extensively investigated in order to obtain further details regarding the chemical structure of the resulting networks. This investigation is not a full characterization of the network structure, however, since silicone elastomers are complex mixtures of polymers and crosslinkers, and potentially also inorganic fillers, resins, chain extenders, and additives. 


\section{WILEY-VCH}

\subsection{Analysis of the chemical characteristics of the elastomers}

FTIR was used to analyze molecular structure and functional groups in the prepared silicone films. FTIR analysis of A and B parts of each formulation was also performed. The FTIR results for the S184_10:1 as well as A and B parts of the Sylgard 184 formulation are shown in Figure 2. While A and B parts of Sylgard 184 and S184_10:1 serve as an example, all formulations investigated in this study showed similar behavior (Data in SI).

The transmittance peaks at $2960 \mathrm{~cm}^{-1}$ were attributed to the $\mathrm{C}-\mathrm{H}$ stretching vibration of the methyl group $\left(-\mathrm{CH}_{3}\right)$. The peaks at $1460 \mathrm{~cm}^{-1}$ were attributed to the $\mathrm{C}=\mathrm{C}$ deformation of $\mathrm{Si}-$ $\mathrm{CH}=\mathrm{CH}_{2}$. Both A and $\mathrm{B}$ parts of the formulation contain PDMS with vinyl functionalities. Furthermore, the presence of the peaks at $1460 \mathrm{~cm}^{-1}$ in the case of S184_10:1 indicates that not all vinyl groups reacted in the hydrosilylation reaction. The peaks at $1255 \mathrm{~cm}^{-1}$ were attributed to the symmetric deformation of $\mathrm{Si}-\mathrm{CH}_{3}$. The strong transmittance peaks at $1000-1100 \mathrm{~cm}^{-1}$ were attributed to the stretching vibrations of $\mathrm{Si}-\mathrm{O}-\mathrm{Si}$. The strong peaks at $800 \mathrm{~cm}^{-1}$ were attributed to the $\mathrm{Si}-\mathrm{C}$ stretching vibrations of $\mathrm{Si}-\left(\mathrm{CH}_{3}\right)_{2}$. All these peaks are characteristic of the PDMS. In the case of part B of Sylgard 184, the distinctive transmittance peaks at 2158 $\mathrm{cm}^{-1}$ and $910 \mathrm{~cm}^{-1}$ were attributed to $\mathrm{Si}-\mathrm{H}$ vibrations, indicating that part $\mathrm{B}$ also contains PDMS with hydride functionality. The disappearance of these Si-H peaks in the case of S184_10:1 indicates that the hydrosilylation reaction was successfully finished. The presence of the $\mathrm{C}=\mathrm{C}$ peaks and disappearance of the Si-H peaks in the case of S184_10:1 indicates an excess of vinyl groups in the formulation.

Peaks at $2158 \mathrm{~cm}^{-1}$ and $910 \mathrm{~cm}^{-1}$ were also observed in the case of part B of the other hard formulation, Sylgard 186 (Data in SI). In case of part B of soft formulations (Ecoflex 00-30 and Ecoflex 00-50), however, the peak at $2158 \mathrm{~cm}^{-1}$ was not observed, while the intensity of the peak at $910 \mathrm{~cm}^{-1}$ was quite low (Data in SI). This indicates a low concentration of hydride groups (below the instrument's detection limit) in the soft formulations, a finding that is in good agreement with their low crosslinking densities. 


\section{WILEY-VCH}

The amount of bonded (gel fraction) species in the networks was determined from swelling experiments with chloroform, the results of which are presented in Table 2. In general, the gel fraction was found to be above approximately $95 \% \mathrm{w} / \mathrm{w}$ for all hard formulation films, and above approximately 52\% w/w for the Eco 50 film. However, the gel fraction was found to be less than $44 \% \mathrm{w} / \mathrm{w}$ for the Eco 30 film. These findings are in good agreement with the elastomers' properties as claimed on their respective data sheets (to be elucidated further in this article). The gel fractions of the blends (MIX Eco 30_S184_3:1 and MIX Eco 50_S184_3:1) were also found to be higher compared to the respective soft formulations (Eco 30 and Eco 50). However, the gel fraction of MIX Eco 50_S184_3:1 film was approximately 7.7\% (relatively) higher than the soft formulation Eco 50 film, while the gel fraction of MIX Eco 30_S184_3:1 was approximately $31 \%$ (relatively) higher than the soft formulation Eco 30. These results suggest that blending soft and hard segments, as in the case of MIX Eco 50_S184_3:1, can induce a small inhibitory effect on the crosslinking efficiency of the two formulations in the blend. This is not surprising, however, since the crosslinking of the blends was not optimized in these studies.

Thermogravimetric analysis of cured silicone formulations was performed from room temperature to $900^{\circ} \mathrm{C}$ under nitrogen atmosphere to inspect general thermal decomposition behavior of the cured silicone elastomer formulations and obtain information on the solid residue after thermal treatment. The onset of thermal degradation is commonly predicted using the temperature at which $5 \%$ weight loss is recorded. ${ }^{[45-47]}$ Onset temperatures of degradation, residual weights at $900^{\circ} \mathrm{C}$, and amounts of filler in each formulation are presented in Table 2. As indicated above, resins and inorganic fillers (most commonly silica fillers) are already present in the silicone elastomer formulations. However, is difficult to determine the amount of resins as well as the type and modification of the fillers already embedded ${ }^{2}$ in a commercial formulation and certainly beyond the scope of this article. The term "filler(s)" will therefore be 


\section{WILEY-VCH}

used throughout the rest of this article to refer to both resins and inorganic fillers present in the silicone elastomers.

S186 formulations showed the highest thermal stability of all investigated silicone formulations, as can be seen from the degradation onset temperatures (Table 2). S186 formulations also showed the highest residual weight at $900^{\circ}$ C. Soft formulations (Eco 30 and Eco 50) were observed to have similar thermal stability and similar residual weight at $900^{\circ} \mathrm{C}$. Both thermal stability and residual weight of soft formulations (Eco 30 and Eco 50) were significantly lower compared to hard formulations (S184 and S186). In the case of MIX Eco 30_S184_3:1, blending of soft and hard segments was observed to decrease thermal stability compared to the respective soft and hard formulations in the blend, as onset temperature of degradation shifted towards lower values. This is most likely caused by the presence of further unreacted groups due to nonstoichiometric crosslinking reaction conditions, as discussed above. The presence of unreacted vinyl has previously been shown to decrease thermal stability. ${ }^{[1]}$ However, the residual weights of blends at $900^{\circ} \mathrm{C}$ resulted in intermediate values, as expected from the combination of formulations with relatively low and high filler contents.

Ogliani et al. ${ }^{[1]}$ reported data from thermogravimetric analysis under nitrogen of pure silicone networks resulting from Pt catalyzed silylation reactions with different crosslinking densities and showed that the residual weight of pure silicone networks at $700^{\circ} \mathrm{C}$ is $0 \%$. Due to the lack of available data on the chemistry of fillers and additives in the formulations investigated here, we assume that the residue of formulations at $900^{\circ} \mathrm{C}$ corresponds to the amount of filler in the formulation.

The hard Sylgard 184 formulation has low viscosity and contains fillers which maintain transparency, while the hard Sylgard 186 formulation and soft formulations (Ecoflex 00-30 and Ecoflex 00-50) have high viscosities and contain relatively high amounts of fillers, affecting transparency and causing their characteristic opacity. As discussed in the Introduction, if optical transparency or low viscosity is needed for a given application, resins are usually added rather 


\section{WILEY-VCH}

than silica. It can therefore be concluded that the Sylgard 184 formulation contains a higher amount of resin compared to Sylgard 186, Ecoflex 00-30, and Ecoflex 00-50 formulations. Data presented in Table 2 shows that, as expected, the hard formulations contain a higher amount of filler than the soft formulations. It was observed that S186 formulations contained higher amounts of filler compared to S184 formulations. The amount of filler is higher in the case of S186_5:1 compared to S186_10:1, indicating that part B of the formulation contains more filler than part A. In the case of S184 formulations, the amount of filler in S184_5:1 is lower compared to S184_10:1, indicating that part A of the formulation has more filler than part B. Furthermore, Eco 30 has a slightly lower amount of filler compared to Eco 50. Our results are in contrast with previous studies indicating that Ecoflex is an unfilled silicone rubber. ${ }^{[41]}$ The blends (MIX Eco 30_S184_3:1 and MIX Eco 50_S184_3:1) were observed to have intermediate filler contents compared to the respective soft and hard formulations used.

A common rule when formulating silicone elastomers is that the Pt catalyst should be kept separate from any hydride functional oligomers and polymers, since the Pt may facilitate condensation of the hydride group over time. One premix therefore usually contains the polymer (vinyl functional) and the catalyst, while the other contains crosslinker mixed with polymer (vinyl functional). The other constituents are mixed into the respective parts depending on their functionalities and their compatibility with $\mathrm{Pt}$ and hydride. The A part of all investigated elastomer formulations in this study can easily be shown to contain the catalyst and polymer, with the $\mathrm{B}$ part containing hydride functional crosslinker and polymer, as confirmed by a simple experiment in which a drop of catalyst and a drop of crosslinker, respectively, were added to the two parts. A parts became solid upon the addition of crosslinker, but not upon addition of catalyst, while B parts behaved oppositely.

1H NMR spectra of A and B parts of Ecoflex 00-30 are presented in Figure 3. 1H NMR spectra of $\mathrm{A}$ and $\mathrm{B}$ parts of Ecoflex 00-50 are similar and are presented in Figure $2 \mathrm{~S}$ in the SI. CDCl3 was used as the solvent for NMR measurements and resulted in a strong signal at $7.25 \mathrm{ppm}$. 


\section{WILEY-VCH}

Methylsilyl groups exhibited strong signals between $-0.25 \mathrm{ppm}$ and $0.5 \mathrm{ppm}$. Signals between $5.68 \mathrm{ppm}$ and $6.19 \mathrm{ppm}$ were assigned to vinyl groups, while signals at $0.88 \mathrm{ppm}$ and $1.21 \mathrm{ppm}$ were assigned to geminate silanol and isolated silanol, respectively. ${ }^{[2]}$ Broad signal at $1.64 \mathrm{ppm}$ was assigned to water, which is most likely introduced via the solvent. ${ }^{[48]}$

Even though FTIR spectra of A and B parts of Ecoflex 00-30 did not indicate the presence of the characteristic hydride peak, 1H NMR spectroscopy revealed that part B contains hydride functionalities, as can be seen from the strong peak at $4.72 \mathrm{ppm}$. This suggests that Ecoflex formulations are likely cross-linked by hydride addition to vinyl groups in the presence of platinum catalyst ${ }^{[49,50]}$. However, the NMR analysis can not verify if there is more than one type of crosslinker, or potentially chain extenders (small, linear polymers with terminal hydride functionalities) as well.

1H NMR spectra of extracts of cured Eco 30 and Eco 50 films showed clear hydride peaks, indicating that not all hydride functions reacted (Figure S3 in SI). This result agrees with extraction experiments, which showed low gel fraction in the films. NMR spectra of Eco 30 and Eco 50 extracts also showed peaks which most likely originate from the Pt-catalyst; this was confirmed by 1H NMR of the Karstedt's catalyst (Figure S3 in SI).

A and B parts of hard formulations Sylgard 184 and Sylgard 186, as well as of soft formulations Ecoflex 00-30 and Ecoflex 00-50, were analyzed by size exclusion chromatography to assess the apparent molecular mass, polydispersity, weight fractions of long chains in each part (A and B) of the formulations, and overall weight fraction of long chains in hard and soft formulations as a whole. These distributions give an indication of the formulation strategy used. Chromatograms of A and B parts of all formulations showed bimodal distributions. A summary of results for all A and B parts is shown in Table 3, along with interpretation of the resulting weight fraction of long polymer chains in the final elastomer mixtures.

Data indicates that both parts of all investigated compositions consist of both long (high molecular weight) and short (low molecular weight) polymer chains. The results can be 


\section{WILEY-VCH}

interpreted as the Sylgard formulations are relatively closely related, with the vinyl functional polymers having a molecular weight in the range of $10-30 \mathrm{~kg} / \mathrm{mol}$ and being crosslinked by low molecular weight crosslinkers, potentially also with chain extenders. The softer of these two formulations (Sylgard 186) is characterized by longer vinyl functional polymers. The low molecular weight species in part A may be a low molecular weight vinyl functional polymer, which produces better properties due to the bimodality of the resulting networks. ${ }^{[51]}$ The Ecoflex formulations show higher molecular weights across all components. The relatively low fractions of long polymer $\left(\mathrm{W}_{\mathrm{f} \text { long chains }}\right)$ indicate a very different formulation strategy from that of the Sylgard formulations. However, their inherent softness indicates a long distance between crosslinking sites, so either they use chain extenders and/or, as indicated by NMR/FTIR, incomplete crosslinking due to nonstoichiometric ratios between hydride and vinyl functional constituents. Summarized findings of different properties of characterized silicone elastomers are presented in Table 4.

\subsection{Ultimate mechanical properties}

Mechanical properties of different formulations obtained by stress-strain measurements, $\mathrm{Y}, \varepsilon_{\mathrm{T}}$, and $\sigma_{\mathrm{T}}$, are presented in Table 5. Based on the value of $\varepsilon_{\mathrm{T}}$ and its standard deviation $\varepsilon_{\mathrm{T}-\mathrm{STD}}$, the maximum tensile strain value, $\varepsilon_{\max }$, up to which different sample formulations were stretched in the cyclic tests without breaking, were defined. In general, $\varepsilon_{\max }$ was chosen as $\varepsilon_{\mathrm{T}-\mathrm{STD}}$; the cycle strain was further reduced for some samples if the given value did not allow for the number of cycles. Data for $\varepsilon_{\mathrm{T}-\mathrm{STD}}$ and $\varepsilon_{\max }$ are presented in Table 5.

S184_5:1 and S184_10:1 were observed to have the highest Young's moduli, Y=2.57 MPa and $\mathrm{Y}=2.26 \mathrm{MPa}$, respectively, but also limited extensibility (113\% and 132\%, respectively). On the other hand, both Eco 30 and Eco 50 had low Young's moduli but high extensibility (Y=0.16 $\mathrm{MPa}$ and $\mathrm{Y}=0.23 \mathrm{MPa} ; 730 \%$ and $749 \%$, respectively). Ecoflex formulations' low Young's moduli and high extensibility indicate that they do not possess a unimodal network structure in which part A contains vinyl terminated long and short polymer chains and the Pt catalyst, while 


\section{WILEY-VCH}

part B contains vinyl terminated long polymer chains and crosslinker agents with hydride or hydride and vinyl terminated crosslinker agents, since a higher modulus and lower extensibility would be expected for such a network. The low Y of Eco 30 and Eco 50 can be increased by blending with $\mathrm{S} 184^{[33,52]}$, which can be seen from the Young's moduli of MIX Eco 30_S184_3:1 and MIX Eco 50_S184_3:1 given in Table 5. As expected, the addition of soft elastomer formulations (Eco 30 and Eco 50) to a hard elastomer formulation (S184_10:1) resulted in intermediate properties. These results are in good agreement with those described in Vaicekauskaite et al. ${ }^{[52]}$ For Eco 50, the resulting tensile strength actually decreased upon "reinforcement" with S184, indicating that the mixture is not ideal seen from a performance point of view, resulting from incompatibility between the two commercial silicone formulations. After performing cyclic uniaxial tension tests up to various stretch levels on all these elastomers, we observed effects related to Mullins softening that were dependent upon the material formulations and the cyclic path ( $1^{\text {st }}$ and $2^{\text {nd }}$ test) followed during these tests.

\subsection{Cyclic uniaxial tension tests}

The stress-strain response of S184_10:1 in both $1^{\text {st }}$ and $2^{\text {nd }}$ tests is presented in Figure 4a) and 4b), respectively. While S184_10:1 serves as an example, all formulations investigated in this study showed similar behavior (Data in SI).

As Figure 4a) shows, constant softening was observed in every first cycle when the maximum strain was increased. Second and third cycle loading curves, closely followed the unloading curve of the first cycle with the same strain. On the other hand, second and third cycle unloading curves showed significantly less softening compared to the first unloading curve within the same strain. In other words, the response became almost entirely elastic after the first cycle. Further, when every subsequent loading surpassed the previous maximum strain, it acted as a continuation of the previous maximum loading curve, indicating that the second and third cycles at the previous strain had little to no influence on the strain history, and thereby again confirming that the second and third cycles were fully elastic. After $\varepsilon_{\max }$ was applied in the $1^{\text {st }}$ 


\section{WILEY-VCH}

test, the material stress-strain response within the whole "strain drop path", closely followed the path of the first $\varepsilon_{\max }$ unloading. In Figure $4 \mathrm{~b}$ ), the same observation is noted for the $2^{\text {nd }}$ test type. In this case, however, all subsequent loading and unloading curves followed the first cycle's unloading curve after $\varepsilon_{\max }$ was applied. Once again, the stress response and softening of soft formulations (Eco 30 and Eco 50; data in SI), similar to the response of the fillerreinforced $^{[41]}$ hard formulations, confirms that Ecoflex is a filled rubber. If Ecoflex were an unfilled rubber, the softening effect would be almost negligible. ${ }^{[24]}$

In both tests, the most pronounced softening resulted after the unloading curve of the $\varepsilon_{\max }$, while significantly less or no softening resulted after all subsequent unloading curves. This finding is confirmed by the determined Young's moduli as function of cycle number, as shown in Figure 5. It can be seen that the most pronounced decrease in $Y$ is after $\varepsilon_{\max }$. In the $1^{\text {st }}$ test of $S 184 \_10: 1$, this decrease occurred after the thirteenth cycle, which corresponds to the first cycle of $\varepsilon_{\max }$ (see Figure 5a)); in the $2^{\text {nd }}$ test of S184_10:1, this decrease occurred after first cycle (see Figure 5b)). Only Eco 50 already showed pronounced softening and decreased $\mathrm{Y}$ at $480 \%$ strain in the "strain rise path" of the $1^{\text {st }}$ test, which is significantly below $\varepsilon_{\max }$ (Data in SI).

It has been reported in the literature ${ }^{[28,31,53]}$ that most significant softening in different materials results after the first unloading cycle. ${ }^{[7,40,53-55]}$ Here, however, we have to account for a difference between the first unloading cycle within each strain and the first unloading cycle within the test as a whole. Figure 5a) shows that when samples were stretched up to strain values above $80 \%$ in the $1^{\text {st }}$ test, significant softening occured after the first unloading cycle within same strain. When samples were stretched to lower strain values (20\% and 40\%), negligable softening occured. On the other hand, the most significant softening observed in the $2^{\text {nd }}$ test resulted after the first unloading cycle within the whole test, while in all subsequent first cycles within same strain there was significantly less or no softening (Figure 5b)).

The stress softening effect can be represented using a strain-energy function. The area under the loading stress-strain curve represents total energy, consisting of inelastic energy (heat 


\section{WILEY-VCH}

disipated energy) and recoverable elastic strain energy. Elastic energy is calculated from the area under the unloading stress-strain curve, and the difference between total and elastic energy represents the inelastic, or disipated, heat energy. ${ }^{[56,57]}$ Total energy and elastic energy values for S184_10:1 in both tests, are plotted in Figures 6a) and 6c), and Figures 6b) and 6d), respectively. Figures 6e) and 6f) present a comparison of the energies in the first three cycles, at $20 \%$ and $40 \%$ strain in the $1^{\text {st }}$ test, and $120 \%$ and $100 \%$ strain in the $2^{\text {nd }}$ test, respectively. Figures 6a)-d) show that both total and elastic energy increased with increasing applied strain in both tests, and decreased with decreasing applied strain. $1^{\text {st }}$ test data (Figures 6a) and 6c)) indicate that total energy values and elastic energy values for low strains (20\% and $40 \%)$, did not change with cycle number within the same strain in the "strain rise path". The same behavior was seen in all cycles following the "strain drop path" (cycle no. 16-27). A comparison between energies at $20 \%$ and $40 \%$ strain in the $1^{\text {st }}$ test showed that their ratio was close to 1 (Figure 6e)), which means that S184_10:1 at low strains acts almost like an ideal elastomer, as discused earlier. However, when strain value was above $80 \%$ in the "strain rise path" (Figure 6a)), the total energy decreased with the cycle number within the same strain, while the decrease in elastic energy was less pronounced (Figure 6c)). A comparison of total and elastic energy values in the second and third cycles of higher strains proves that response in these cycles was almost entirely elastic. Both total and elastic energy had higher values in the "strain rise path" compared to the "strain drop path" at the same applied strain. Additionally, the difference between total and elastic energy in the "strain rise path" was higher than the difference between these energies in the "strain drop path". This indicates that most of the disipated energy in the $1^{\text {st }}$ test derives from the "strain rise path".

$2^{\text {nd }}$ test data (Figures $6 \mathrm{~b}$ ) and $6 \mathrm{~d}$ )) indicate that total energy experienced the largest decrease after the first cycle, while the decrease in elastic energy was less pronounced. After the first cycle, the change in total and elastic energy within the same strain was negligable. A comparison between energies after the first cycle shows a ratio close to 1 (Figure 6f)), which 


\section{WILEY-VCH}

means that S184_10:1 starts to act almost like an ideal elastomer after $\varepsilon_{\max }$ is applied. Both total energy and elastic energy had similar values in the "strain rise path" and "strain drop path" within the same applied strain, except in the first cycle at $\varepsilon_{\max }$. This indicates that the largest energy dissipation in the $2^{\text {nd }}$ test occurs in the first cycle. It is therefore clear that the silicone elastomer formulations exhibit idealized Mullins behavior after the first cycle in the $2^{\text {nd }}$ test, since the softening effect is negligible in all subsequent deformation cycles. ${ }^{[20]}$ This suggests that the softening effect can be avoided in silicone elastomer forumulations by prestretching the sample to $\varepsilon_{\max }$ of the particular formulation (or, alternatively, beyound the maximum strain envisioned for the product) one time before the elastomer is employed at the given product.

The strain energy function of the $1^{\text {st }}$ test can be used to quantify the Mullins effect in the different commercial formulations. Since the largest energy dissipation occurs in every first cycle in the strain rise path of the $1^{\text {st }}$ test, the dissipated energy, normalized by the total energy in every first cycle, can be used as a measure of the softening effect at different strains. Different formulations can be compared via the softening effect at identical applied strains. However, this comparison between different formulations at the same applied strain may lead to incorrect conclusions, since the stresses developed in the elastomer will be very different at an absolute strain, of e.g., 20\%. For example, the applied strain in the first cycle of the $1^{\text {st }}$ test is $20 \%$, which, in the case of hard formulations (S184 and S186), is 5 and 17 times lower, respectively, than the maximum strain that these formulations can sustain without breaking, but is 34 times lower in the case of soft formulations.

Figure 7 presents the dissipated energy normalized by the total energy for different formulations in the first cycle of the $1^{\text {st }}$ test at a strain of approximately $20 \%$ of $\varepsilon_{\mathrm{T}}$ for each formulation. This measure enables comparison of the softening effect in formulations at the same strain level relative to $\varepsilon_{\mathrm{T}}$. Data presented in Figure 7 shows that hard formulation S184_10:1 has a lower softening effect compared to S186_10:1, likely due to its lower filler content. The softening effect also decreased in S184_5:1 and S186_5:1 compared to S184_10:1 


\section{WILEY-VCH}

and S186_10:1, respectively. This decrease is as expected for S184_5:1, which contains less filler than S184_10:1. However, S186_5:1 has a higher filler content than S186_10:1; yet both S184_5:1 and S186_5:1 formulations contain higher percentege of part B compared to S184_10:1 and S186_10:1. Since part B of the elastomer formulations contains functionalized polymer chains as crosslinker, formulations with a 5:1 ratio of A and B parts contain a larger number of crosslinking points compared to formulations with 10:1. These results suggest that a highly crosslinked network with higher concentration of crosslinking points experiences less reorganisation and less sliding of fillers, as well as less chain slippage, resulting in a reduced softening effect compared to networks with lower concentrations of crosslinking points. Unsurprisingly, then, Eco 50 and blends (MIX Eco 30_S184_3:1 and MIX Eco 50_S184_3:1) exhibit a lower softening effect compared to hard formulations. SEC data indicates that all formulations consist of both high and low molecular weight polymer chains. However, soft formulations' polymer chains have significantly higher molecular weights compared to those of hard formulations. This is also expected, since elasticity scales with crosslinking density, which again scales inversely to the molecular weight of the polymer. TGA data also show that the soft formulations contain less filler compared to hard formulations, suggesting that this lower filler content is one reason for the lower softening effect in soft formulations compared to hard. The synergetic effect of long and short polymer chains in the soft formulations may also contribute to soft formulations and blends' comparatively reduced softening effect in relation to purely hard formulations. ${ }^{[58]}$ Both long and short polymer chains in soft formulations are significantly longer compared to those in hard formulations. The unexpectedly high softening effect observed in case of Eco 30 compared to all other formulations may be a result of the relatively high sol fraction in Eco 30 films. All polymer chains are initially stretched under low applied strain, contributing to overall stress, ${ }^{[58]}$ upon increased strain, however, the sol fraction polymer chains will relax their stresses and not contribute further to the overall stress. At 120\% strain and below, Eco 30 displays a higher softening effect than Eco 50. 


\section{WILEY-VCH}

However, this situation reverses at higher applied strains (See Figure 47S in SI). Blends MIX Eco 30_S184 and MIX Eco 50_S184 have lower softening effect compared to the soft formulations (Eco 30 and Eco 50). Results show that blending increases the reversibility of the sample geometry compared to both soft and hard formulations used in the blend, as expected due to the increased crosslinker content compared to the soft formulations. Results also show that the Mullins effect is indeed more pronounced in elastomers which contain higher amounts of filler ${ }^{[24]}$ (hard formulations). However, in contrast to the common understanding that the Mullins effect increases with increasing filler content (hardness), our results show that the Mullins effect actually becomes more prominent when the elastomer network becomes very soft. This indicates that the Mullins effect is not only impacted by the presence of fillers, but also by the number of bonded species in the network (gel fraction), number of crosslinking points, and the molecular weight of polymer chains. This further suggests that the softening effect may be limited by choosing a silicone elastomer formulation with low filler content, high molecular weight polymer chains, and a high number of crosslinking functionalities, thereby ensuring that a network with low sol fraction is obtained.

Residual extension of the material remaining after subjection to the cyclic uniaxial test, known as permanent set, was also observed in some formulations. In the case of soft formulations Eco 30 and Eco 50, as well as their blends with S184_10:1, permanent set was observed after stretching samples to $\varepsilon_{\max }$; permanent residual extension of the elastomer was then visually confirmed by comparing the deformed sample with a non-deformed (pristine) one after the test is finished (Figure 8a)). Permanent set was not observed in Sylgard 184 and Sylgard 186 formulations. Examples of a deformed S184_10:1 sample and a non-deformed S184_10:1 sample are presented in Figure 8b).

All mechanical test methods reported here are in agreement with previous studies ${ }^{[20,35,38,41,42,59-}$ ${ }^{61]}$ on the Mullins effect in elastomers, with some differences. Previous studies used either rectangular shaped specimens ${ }^{[38,41,60,61]}$ or different dog bone shaped specimens ${ }^{[35,42,59]}$. These 


\section{WILEY-VCH}

studies also reported different strain rates, number of cycles, and maximum applied strains, all of which affect results. Reported maximum applied strains of soft formulation samples (Eco 30 and Eco 50) in uniaxial cyclic tests are significantly lower than the maximum applied strains in this work. ${ }^{[38,41]}$ The higher extensibility of the dog bone shaped elastomer samples reported here is expected, since the dog bone geometry is used to eliminate high stress concentrations. Furthermore, previous studies did not report test methods containing both "strain rise" and "strain drop" paths. ${ }^{[35,38,41,59-61]}$

\section{Conclusion}

We investigated the Mullins effect in four commercial silicone formulations (Sylgard 184, Sylgard 186, Ecoflex 00-30 and Ecoflex 00-50), two combinations of two commercial silicone formulations (Sylgard 184 and Ecoflex 00-30, Sylgard 184 and Ecoflex 00-50) and two silicone formulations mixed at different stoichiometric ratios (Sylgard 184 and Sylgard 186). Two types of cyclic uniaxial tension tests were performed, and results showed that the point of softening greatly depends on both the elastomer formulation and strain history. Young's moduli, total energy and elastic energy were determined for every cycle. In the $1^{\text {st }}$ test, constant softening was observed in every first cycle, with significantly less or no softening in the second and third cycles within the same strain. In both tests, the most pronounced softening resulted from the unloading curve of the $\varepsilon_{\max }$, which occurs in the first cycle of the $2^{\text {nd }}$ test. Significantly less or no softening resulted from all subsequent unloading curves. In other words, elastomers` response is almost entirely elastic after $\varepsilon_{\max }$ is applied. Total and elastic energy values indicate that the largest energy dissipation in the $1^{\text {st }}$ test occurs within the "strain rise path", while in the $2^{\text {nd }}$ test the maximum energy dissipation occurs in the first cycle. Dissipated energy normalized by the total energy in the first cycle of the $1^{\text {st }}$ test at approximately $20 \%$ of $\varepsilon_{\mathrm{T}}$ of each formulations enabled comparison of softening effect in different formulations. Mullins effect is significant in the formulations which contain high amount of fillers. Moreover, data indicates that when the elastomer network becomes very soft the Mullins effect becomes more 


\section{WILEY-VCH}

pronounced. Significant permanent set was observed in the softest commercial formulations which reveals that it is not just the filler content that dictates the Mullins effect, but rather a complicated interplay between the fillers and the covalent network structure.

\section{Supporting Information}

Supporting Information is available from the Wiley Online Library or from the author.

\section{Acknowledgements}

Financial support from the Hempel Foundation to CoaST (The Hempel Foundation Coatings Science and Technology Centre) is acknowledged. Authors would like to thank senior researcher Liyun Yu at DTU for performing TGA and DSC measurements.

Received: ((will be filled in by the editorial staff))

Revised: ((will be filled in by the editorial staff)) Published online: ((will be filled in by the editorial staff))

\section{References}

[1] Ogliani, E.; Yu, L.; Mazurek, P.; Skov, A. L. Designing Reliable Silicone Elastomers for High-Temperature Applications. Polym. Degrad. Stab. 2018, 157, 175-180.

[2] Delebecq, E.; Ganachaud, F. Looking over Liquid Silicone Rubbers: (1) Network Topology vs Chemical Formulations. ACS Appl. Mater. Interfaces 2012, 4 (7), 3340-3352.

[3] Bauer, S.; Bauer-Gogonea, S.; Graz, I.; Kaltenbrunner, M.; Keplinger, C.; Schwödiauer, R. 25th Anniversary Article: A Soft Future: From Robots and Sensor Skin to Energy Harvesters. Adv. Mater. 2014, $26(1), 149-162$.

[4] Wallin, T. J.; Pikul, J.; Shepherd, R. F. 3D Printing of Soft Robotic Systems. Nat. Rev. Mater. 2018, 3 (6), 84-100.

[5] Arruda, E. M.; Boyce, M. C. A Three-Dimensional Constitutive Model for the Large Stretch Behavior of Rubber Elastic Materials. J. Mech. Phys. Solids 1993, 41 (2), 389-412.

[6] Ciferri, A.; Hoeve, C. A. J.; Flory, P. J. Stress-Temperature Coefficients of Polymer Networks and the Conformational Energy of Polymer Chains. J. Am. Chem. Soc. 1961, 83 (5), $1015-1022$. 


\section{WILEY-VCH}

[7] MacHado, G.; Chagnon, G.; Favier, D. Induced Anisotropy by the Mullins Effect in Filled Silicone Rubber. Mech. Mater. 2012, 50, 70-80.

[8] Mullins, L. Effect of Stretching on the Properties of Rubber. Rubber Chem. Technol. 1948, 21 (2), 281-300.

[9] Tobin.N.R; Mullins.L. Theoretical Model for the Elastic Behavior of Filler-Reinforced Vulcanized Rubbers. Rubber Chem. Technol. 1957, 30 (20), 555-571.

[10] Winter, H. H.; Chambon, F. Analysis of Linear Viscoelasticity of a Crosslinking Polymer at the Gel Point. J. Rheol. (N. Y. N. Y). 1986, 30 (2), 367-382.

[11] Larsen, A. L.; Hansen, K.; Sommer-Larsen, P.; Hassager, O.; Bach, A.; Ndoni, S.; Jørgensen, M. Elastic Properties of Nonstoichiometric Reacted PDMS Networks. Macromolecules 2003, 36 (26), 10063-10070.

[12] Zhong, M.; Wang, R.; Kawamoto, K.; Olsen, B. D.; Johnson, J. A. Quantifying the Impact of Molecular Defects on Polymer Network Elasticity. Polym. Sci. 2016, 353 (6305), $1264-1268$.

[13] Fetters, L. J.; Lohse, D. J.; Richter, D.; Witten, T. A.; Zirkel, A. Connection between Polymer Molecular Weight, Density, Chain Dimensions, and Melt Viscoelastic Properties. Macromolecules 1994, 27 (17), 4639-4647.

[14] Neuefeind, J.; Skov, A. L.; Daniels, J. E.; Honkimäki, V.; Jakobsen, B.; Oddershede, J.; Poulsen, H. F. A Multiple Length Scale Description of the Mechanism of Elastomer Stretching. RSC Adv. 2016, 6 (98), 95910-95919.

[15] Abadi, M.; Serag, M. F.; Habuchi, S. Entangled Polymer Dynamics beyond Reptation. Nat. Commun. 2018, $9(1), 1-12$.

[16] Dominique, B.; Jean-Yves, B.; Pollock, T.; Poulsen, H. F.; Rollett A., U. M. Proceedings of the 2nd International Congress on 3D Materials Science; Springer International Publishers, Switzerland: Annecy, 2016; p 142. 


\section{WILEY-VCH}

[17] Frankær, S. M. G.; Jensen, M. K.; Bejenariu, A. G.; Skov, A. L. Investigation of the Properties of Fully Reacted Unstoichiometric Polydimethylsiloxane Networks and Their Extracted Network Fractions. Rheol. Acta 2012, 51 (6), 559-567.

[18] Rothon, R. Fillers for Polymer Applications; Springer International Publishers, Switzerland, 2017.

[19] Mazurek, P.; Vudayagiri, S.; Skov, A. L. How to Tailor Flexible Silicone Elastomers with Mechanical Integrity: A Tutorial Review. Chem. Soc. Rev. 2019, 48 (6), 1448-1464.

[20] Dargazany, R.; Itskov, M. Constitutive Modeling of the Mullins Effect and Cyclic Stress Softening in Filled Elastomers. Phys. Rev. E - Stat. Nonlinear, Soft Matter Phys. 2013, 88 (1), $1-13$.

[21] Mullins, L. Softening of Rubber by Deformation. Rubber Chem. Technol. 1969, 42 (1), 339-362.

[22] Rey, T.; Chagnon, G.; Le Cam, J. B.; Favier, D. Influence of the Temperature on the Mechanical Behaviour of Filled and Unfilled Silicone Rubbers. Polym. Test. 2013, 32 (3), 492501.

[23] Zhong, D.; Xiang, Y.; Yin, T.; Yu, H.; Qu, S.; Yang, W. A Physically-Based Damage Model for Soft Elastomeric Materials with Anisotropic Mullins Effect. Int. J. Solids Struct. 2019, 176-177, 121-134.

[24] Dargazany, R.; Itskov, M. A Network Evolution Model for the Anisotropic Mullins Effect in Carbon Black Filled Rubbers. Int. J. Solids Struct. 2009, 46 (16), 2967-2977.

[25] Bueche, F. Molecular Basis for the Mullins Effect. J. Appl. Polym. Sci. 1960, 4 (10), 107-114.

[26] Bueche, F. Mullins Effect and Rubber-Filler Interaction. J. Appl. Polym. Sci. 1961, 5 (15), 271-281.

[27] Blanchard, A. F.; Parkinson, D. Breakage of Carbon-Rubber Networks by Applied 


\section{WILEY-VCH}

Stress. Ind. Eng. Chem. 1952, 44 (4), 799-812.

[28] Cantournet, S.; Desmorat, R.; Besson, J. Mullins Effect and Cyclic Stress Softening of Filled Elastomers by Internal Sliding and Friction Thermodynamics Model. Int. J. Solids Struct. 2009, 46 (11-12), 2255-2264.

[29] Kraus, G.; Childers, C. W.; Rollmann, K. W. Stress Softening in Carbon Blackreinforced Vulcanizates. Strain Rate and Temperature Effects. J. Appl. Polym. Sci. 1966, 10 (2), 229-244.

[30] Hanson, D. E.; Hawley, M.; Houlton, R.; Chitanvis, K.; Rae, P.; Orler, E. B.; Wrobleski, D. A. Stress Softening Experiments in Silica-Filled Polydimethylsiloxane Provide Insight into a Mechanism for the Mullins Effect. Polymer (Guildf). 2005, 46 (24), 10989-10995.

[31] Maiti, A.; Small, W.; Gee, R. H.; Weisgraber, T. H.; Chinn, S. C.; Wilson, T. S.; Maxwell, R. S. Mullins Effect in a Filled Elastomer under Uniaxial Tension. Phys. Rev. E - Stat. Nonlinear, Soft Matter Phys. 2014, 89 (1), 1-6.

[32] Atalay, A.; Sanchez, V.; Atalay, O.; Vogt, D. M.; Haufe, F.; Wood, R. J.; Walsh, C. J. Batch Fabrication of Customizable Silicone-Textile Composite Capacitive Strain Sensors for Human Motion Tracking. Adv. Mater. Technol. 2017, 2 (9).

[33] Park, S.; Mondal, K.; Treadway, R. M.; Kumar, V.; Ma, S.; Holbery, J. D.; Dickey, M. D. Silicones for Stretchable and Durable Soft Devices: Beyond Sylgard-184. ACS Appl. Mater. Interfaces 2018, 10 (13), 11261-11268.

[34] Kwon, D.; Lee, T. I.; Shim, J.; Ryu, S.; Kim, M. S.; Kim, S.; Kim, T. S.; Park, I. Highly Sensitive, Flexible, and Wearable Pressure Sensor Based on a Giant Piezocapacitive Effect of Three-Dimensional Microporous Elastomeric Dielectric Layer. ACS Appl. Mater. Interfaces 2016, 8 (26), 16922-16931.

[35] Case, J. C.; White, E. L.; Kramer, R. K. Soft Material Characterization for Robotic Applications. Soft Robot. 2015, 2 (2), 80-87. 


\section{WILEY-VCH}

[36] Kenry; Yeo, J. C.; Lim, C. T. Emerging Flexible and Wearable Physical Sensing Platforms for Healthcare and Biomedical Applications. Microsystems Nanoeng. 2016, 2 (April).

[37] MacHado, G.; Chagnon, G.; Favier, D. Analysis of the Isotropic Models of the Mullins Effect Based on Filled Silicone Rubber Experimental Results. Mech. Mater. 2010, 42 (9), 841851.

[38] Liao, Z.; Hossain, M.; Yao, X.; Navaratne, R.; Chagnon, G. A Comprehensive ThermoViscoelastic Experimental Investigation of Ecoflex Polymer. Polym. Test. 2020, 86 (January), 106478.

[39] Schneider, F.; Fellner, T.; Wilde, J.; Wallrabe, U. Mechanical Properties of Silicones for MEMS. J. Micromechanics Microengineering 2008, 18 (6).

[40] Roland C.M. The Mullins Effect in Crosslinked Rubber. J. Rheol. (N. Y. N. Y). 1989, 33 (4), 659-670.

[41] Liao, Z.; Hossain, M.; Yao, X. Ecoflex Polymer of Different Shore Hardnesses: Experimental Investigations and Constitutive Modelling. Mech. Mater. 2020, 144 (January).

[42] Steck, D.; Qu, J.; Kordmahale, S. B.; Tscharnuter, D.; Muliana, A.; Kameoka, J. Mechanical Responses of Ecoflex Silicone Rubber: Compressible and Incompressible Behaviors. J. Appl. Polym. Sci. 2019, 136 (5), 1-11.

[43] Zakaria, S.; Madsen, F. B.; Skov, A. L. Post Curing as an Effective Means of Ensuring the Long-Term Reliability of PDMS Thin Films for Dielectric Elastomer Applications. Polym. Plast. Technol. Eng. 2017, 56 (1), 83-95.

[44] Madsen, P. J.; Yu, L.; Boucher, S.; Skov, A. L. Enhancing the Electro-Mechanical Properties of Polydimethylsiloxane Elastomers through Blending with Poly(DimethylsiloxaneCo-Methylphenylsiloxane) Copolymers. RSC Adv. 2018, 8 (41), 23077-23088.

[45] Hanu, L. G.; Simon, G. P.; Cheng, Y. B. Thermal Stability and Flammability of Silicone Polymer Composites. Polym. Degrad. Stab. 2006, 91 (6), 1373-1379. 


\section{WILEY-VCH}

[46] Gornicka, B.; Gorecki, L. TGA/DTG/DSC Investigation of Thermal Ageing Effects on Polyamide-Imide Enamel. J. Therm. Anal. Calorim. 2010, 101 (2), 647-650.

[47] Hanu, L. G.; Simon, O. P.; Mansouri, J.; Burford, R. P.; Cheng, Y. B. Development of Polymer-Ceramic Composites for Improved Fire Resistance. J. Mater. Process. Technol. 2004, 153-154 (1-3), 401-407.

[48] Fulmer, G. R.; Miller, A. J. M.; Sherden, N. H.; Gottlieb, H. E.; Nudelman, A.; Stoltz, B. M.; Bercaw, J. E.; Goldberg, K. I. NMR Chemical Shifts of Trace Impurities: Common Laboratory Solvents, Organics, and Gases in Deuterated Solvents Relevant to the Organometallic Chemist. Organometallics 2010, 29 (9), 2176-2179.

[49] Lewis, L. N.; Stein, J.; Gao, Y.; Colborn, R. E.; Hutchins, G. Platinum Catalysts Used in the Silicones Industry: Their Synthesis and Activity in Hydrosilylation. Platin. Met. Rev. 1997, $41(2), 66-75$.

[50] Lewis, L. N.; Lewis, N. Platinum-Catalyzed Hydrosilylation-Colloid Formation as the Essential Step. J. Am. Chem. Soc. 1986, 108 (23), 7228-7231.

[51] Bejenariu, A. G.; Yu, L.; Skov, A. L. Low Moduli Elastomers with Low Viscous Dissipation. Soft Matter 2012, 8 (14), 3917-3923.

[52] Vaicekauskaite, J.; Mazurek, P.; Vudayagiri, S.; Skov, A. L. Mapping the Mechanical and Electrical Properties of Commercial Silicone Elastomer Formulations for Stretchable Transducers. J. Mater. Chem. C 2020, 8 (4), 1273-1279.

[53] Diani, J.; Fayolle, B.; Gilormini, P. A Review on the Mullins Effect. Eur. Polym. J. 2009, $45(3), 601-612$.

[54] Harwood, J. A. C.; Payne, A. R. Stress Softening in Natural Rubber Vulcanizates. Part III. Carbon Black-filled Vulcanizates. J. Appl. Polym. Sci. 1966, 10 (2), 315-324.

[55] Harwood, J. A. C.; Mullins, L.; Payne, A. R. Stress Softening in Natural Rubber Vulcanizates. Part II. Stress Softening Effects in Pure Gum and Filler Loaded Rubbers. J. Appl. 


\section{WILEY-VCH}

Polym. Sci. 1965, 9 (9), 3011-3021.

[56] Ogden, R. W.; Roxburgh, D. G. A Pseudo-Elastic Model for the Mullins Effect in Filled Rubber. Proc. R. Soc. A Math. Phys. Eng. Sci. 1999, 455 (1988), 2861-2877.

[57] Dorfmann, A.; Ogden, R. W. A Constitutive Model for the Mullins Effect with Permanent Set in Particle-Reinforced Rubber. Int. J. Solids Struct. 2004, 41 (7), 1855-1878.

[58] Madsen, F. B.; Daugaard, A. E.; Fleury, C.; Hvilsted, S.; Skov, A. L. Visualisation and Characterisation of Heterogeneous Bimodal PDMS Networks. RSC Adv. 2014, 4 (14), 6939_ 6945.

[59] Samaca Martinez, J. R.; Le Cam, J. B.; Balandraud, X.; Toussaint, E.; Caillard, J. New Elements Concerning the Mullins Effect: A Thermomechanical Analysis. Eur. Polym. J. 2014, $55(1), 98-107$.

[60] Diaz, R.; Diani, J.; Gilormini, P. Physical Interpretation of the Mullins Softening in a Carbon-Black Filled SBR. Polymer (Guildf). 2014, 55 (19), 4942-4947.

[61] Merckel, Y.; Diani, J.; Brieu, M.; Caillard, J. Constitutive Modeling of the Anisotropic Behavior of Mullins Softened Filled Rubbers. Mech. Mater. 2013, 57, 30-41.

a)

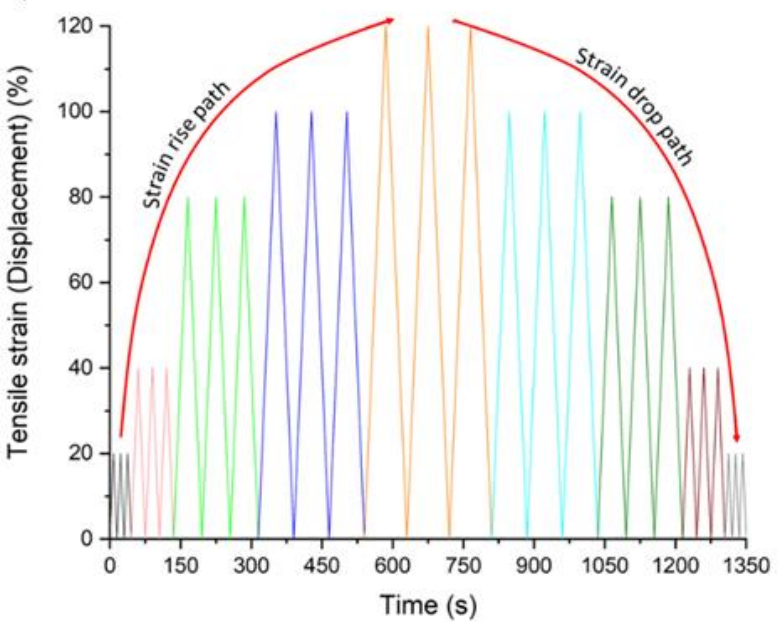

b)

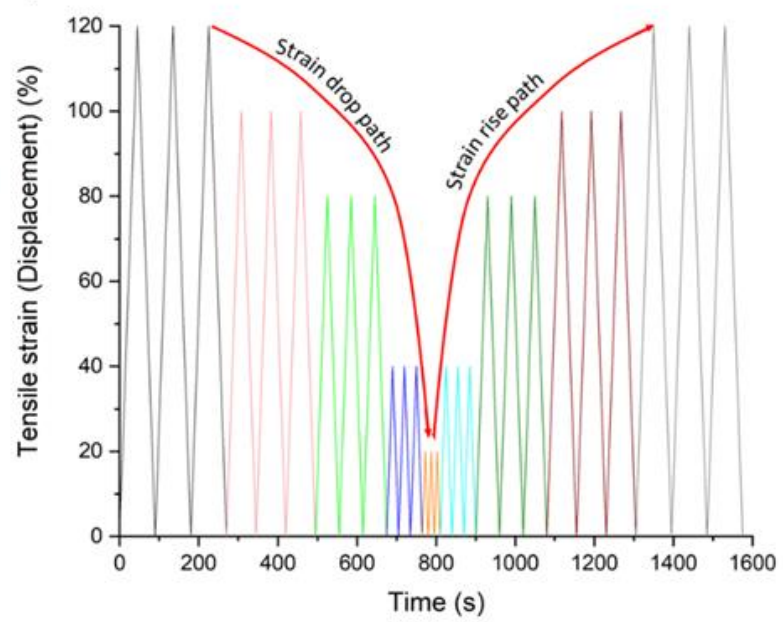

Figure 1. Schematic illustration of the first a) and second b) experimental test. The time scales in the two figures vary slightly due to different number of cycles at highest and lowest strains. 


\section{WILEY-VCH}

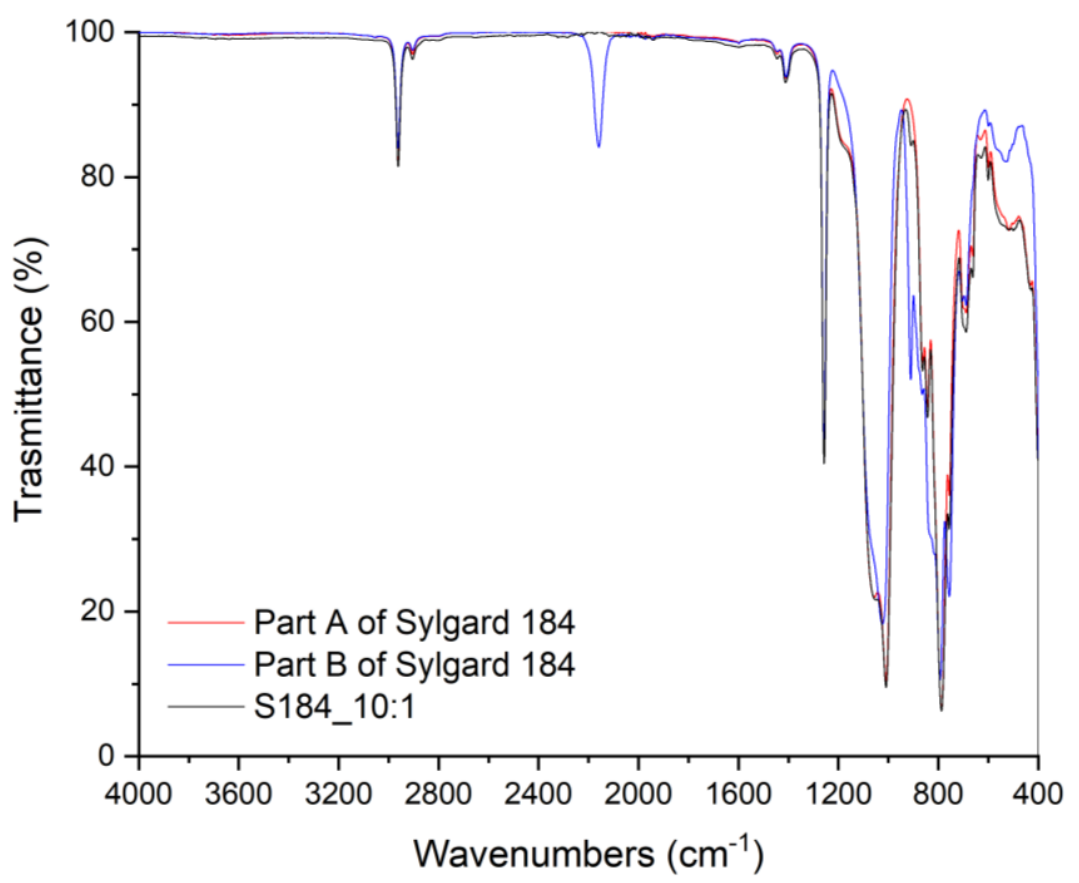

Figure 2. FTIR spectra of part A (red) and part B (blue) of Sylgard 184 formulation, and the final elastomer S184_10:1 sample (black).

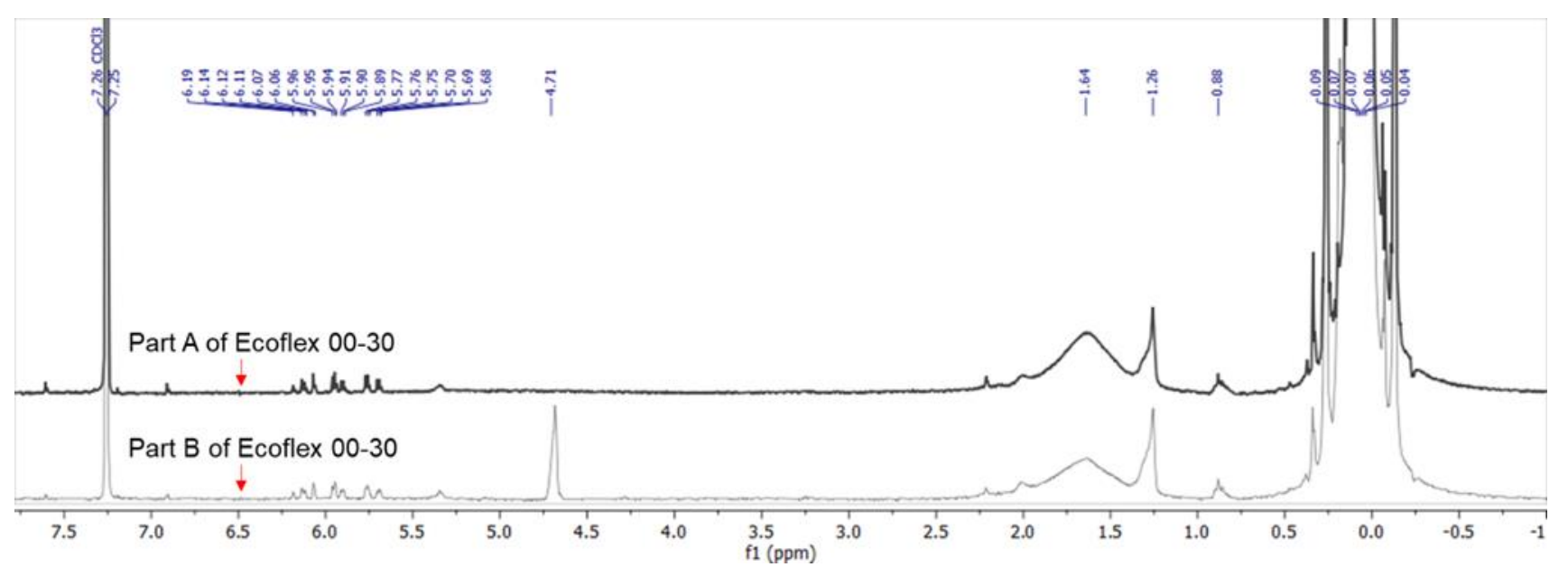

Figure 3. 1H NMR spectra of part A (black line) and part B (grey line) of Ecoflex 00-30.

a)

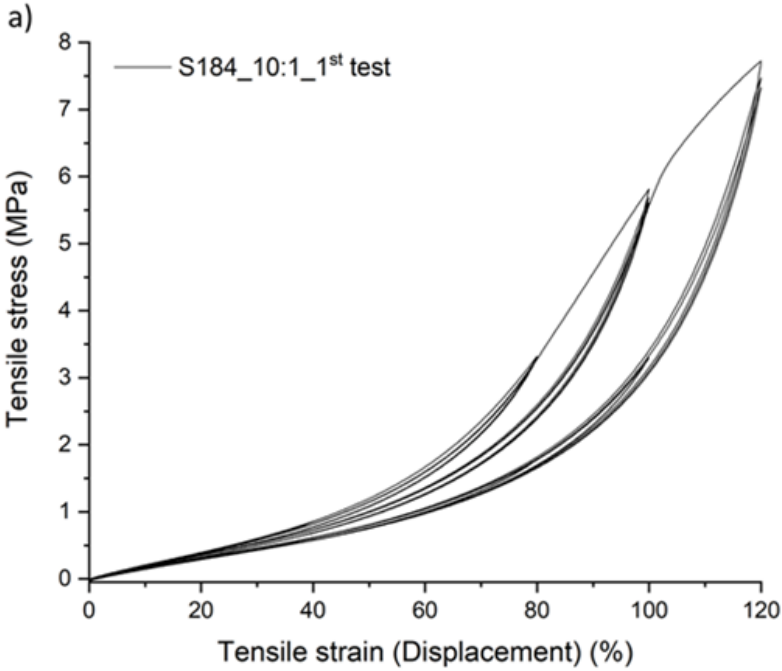

b)

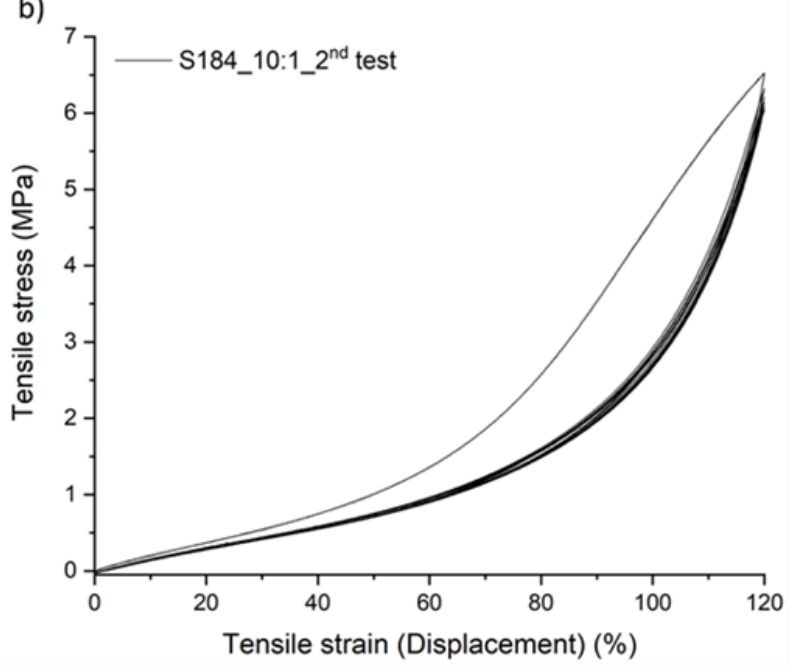




\section{WILEY-VCH}

Figure 4. Stress-strain response of S184_10:1 submitted to a) $1^{\text {st }}$ test, in which cycle strain is gradually increased, and b) $2^{\text {nd }}$ test, in which sample is subjected to maximum strain first. Tests were performed with $2 \mathrm{~mm} / \mathrm{s}$ strain rate and at room temperature $\left(23^{\circ} \mathrm{C}\right)$.

a)

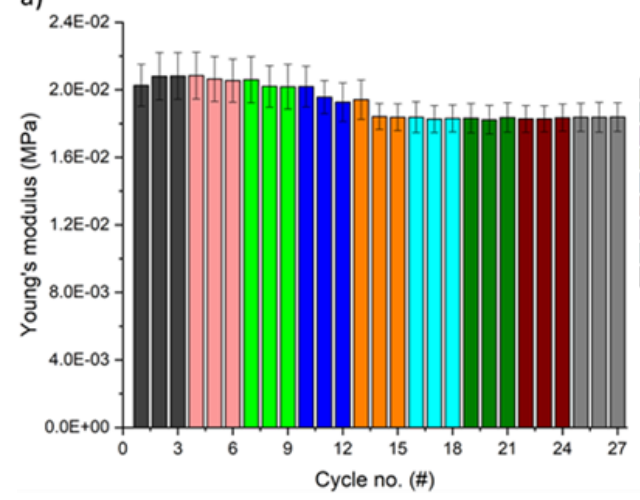

b)

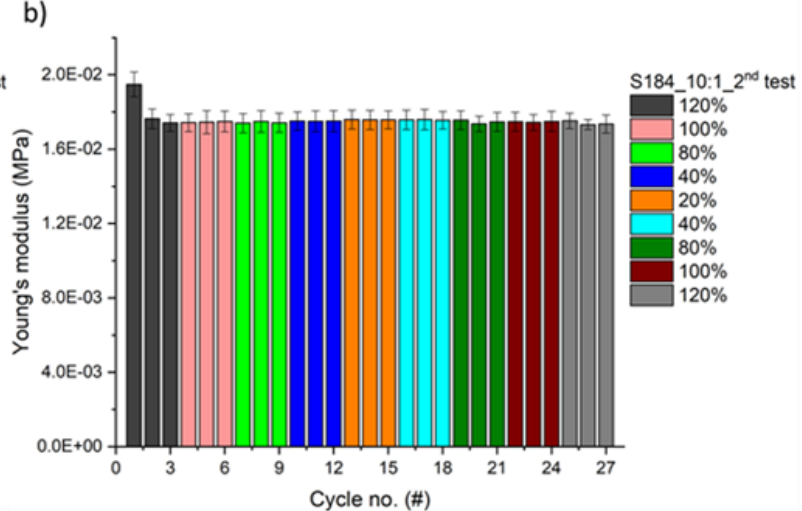

Figure 5. Young's modulus (at 10\% strain) as a function of cycle number for different strains for S184_10:1 subjected to a) $1^{\text {st }}$ test, and b) $2^{\text {nd }}$ test. After cycles $13-15$, the strain path was reversed. 


\section{WILEY-VCH}
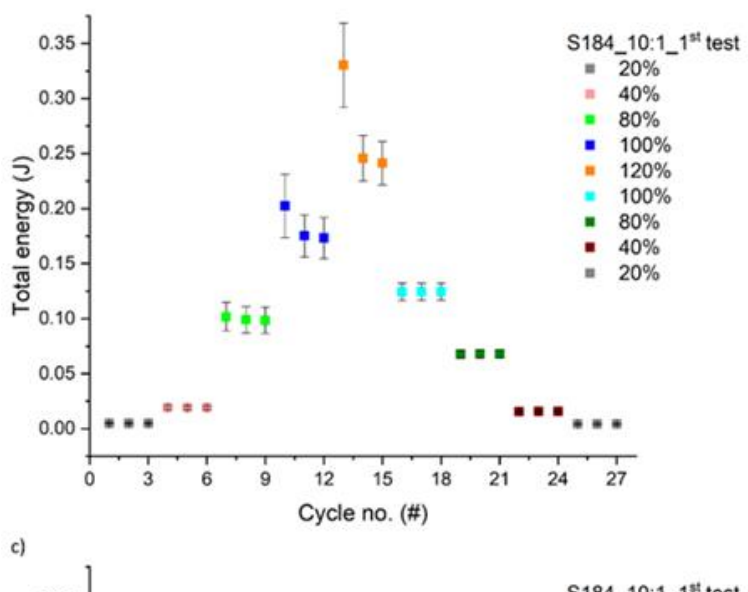

b)

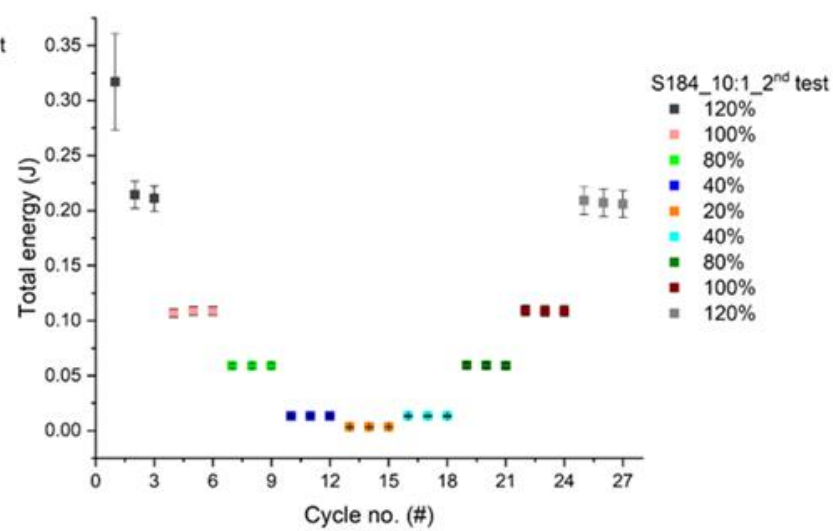

d)
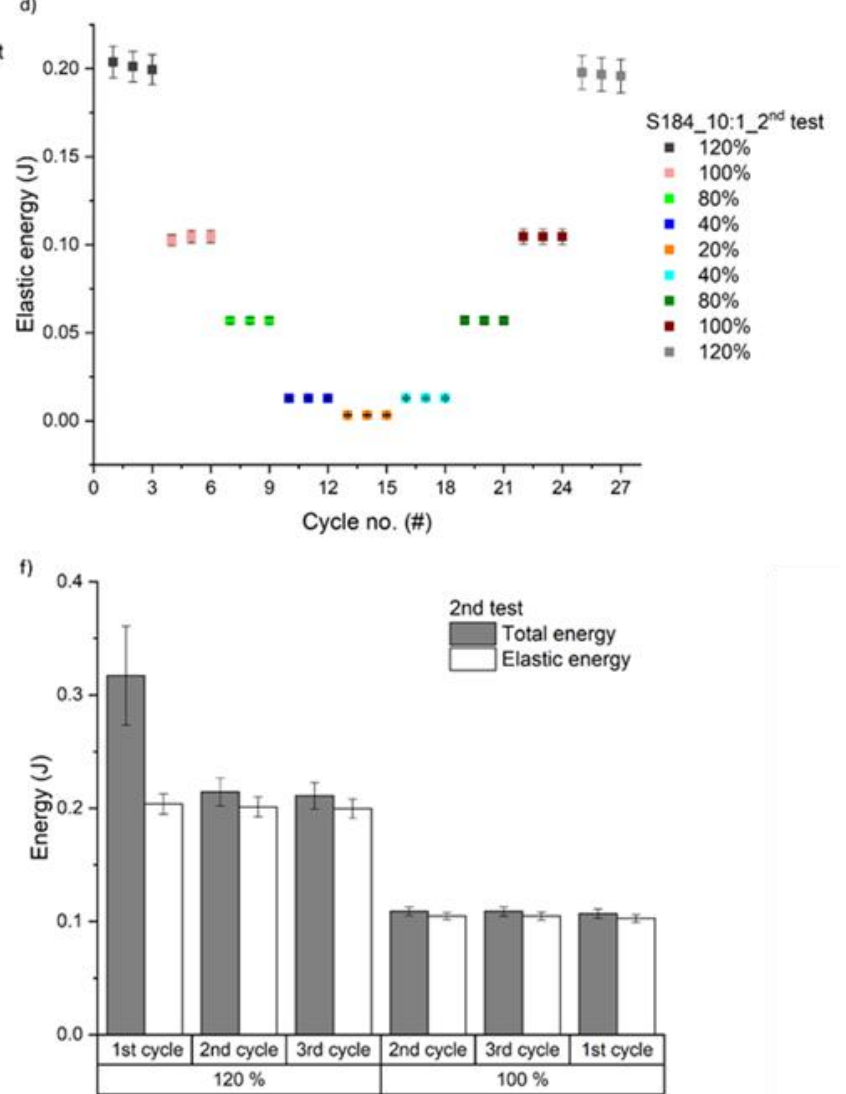

Figure 6. Total energy a), b) and elastic energy c), d) as a function of cycle number for different strains of S184_10:1 submitted to a $1^{\text {st }}$ test a), c) and $2^{\text {nd }}$ test b), d). After cycles 13-15, the strain was reversed. Total energy and Elastic energy of S184_10:1 in the first six cycles of the e) $1^{\text {st }}$ test and f) $2^{\text {nd }}$ test. 


\section{WILEY-VCH}

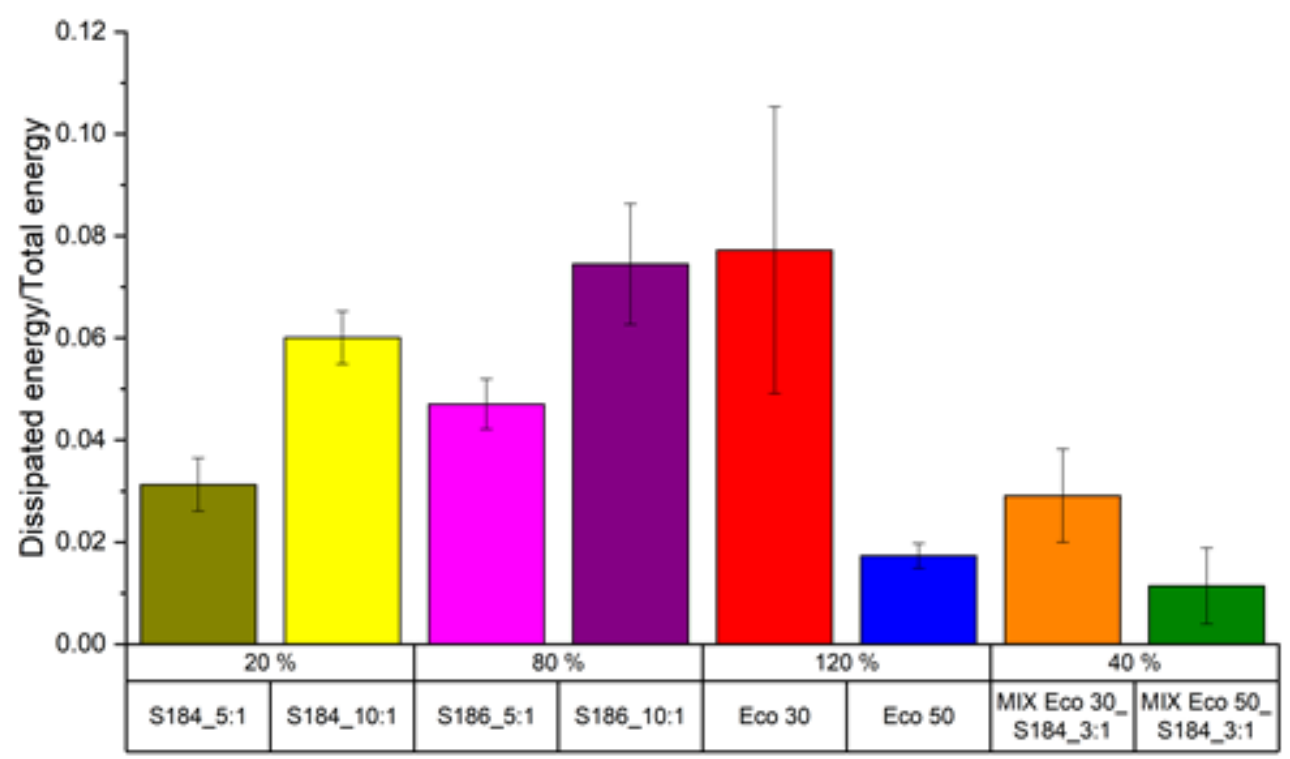

Figure 7. Dissipated energy normalized by the total energy of different silicone formulations in the first cycle of the $1^{\text {st }}$ test at approximately $20 \%$ of strain at break.

a)
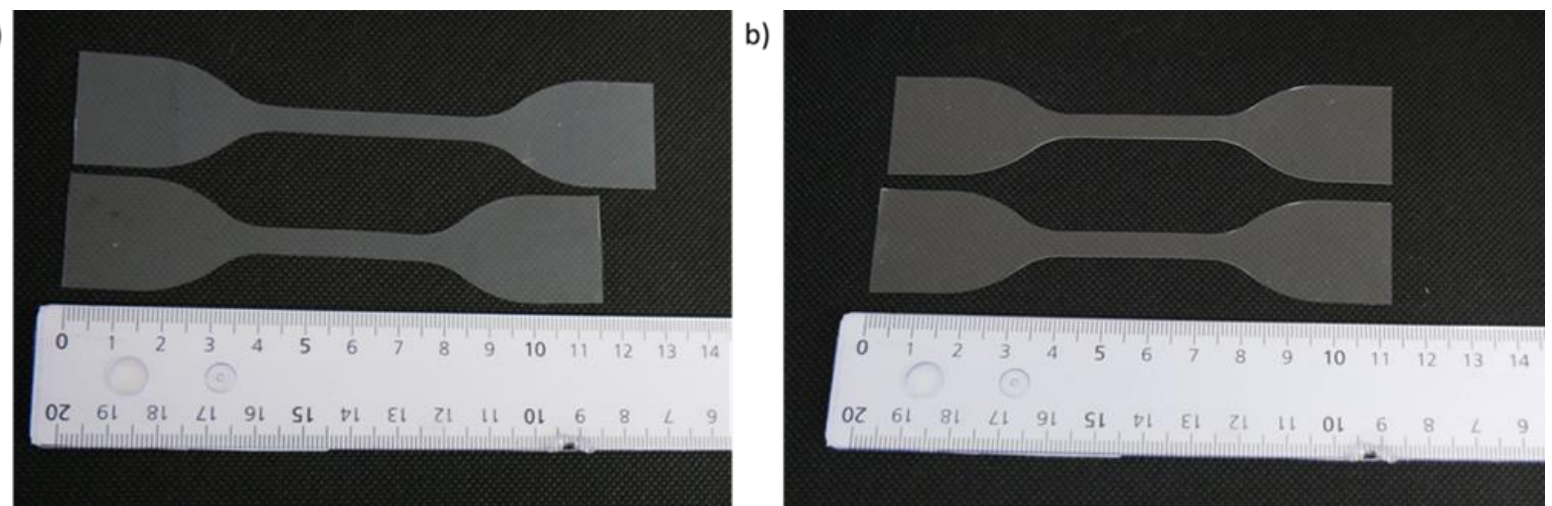

Figure 8. Samples of a) Eco 50 and b) S184_10:1 before (bottom specimens) and after (top specimens) cyclic uniaxial tension test.

Table 1. Sample names with their corresponding mixing ratios (part A and part B); blending ratios with other formulations.

\begin{tabular}{|c|c|c|c|c|c|}
\hline No. & Sample name & Part I* & Ratio & Part II* & Ratio \\
\hline 1 & S184_5:1 & Sylgard $184(5: 1)$ & - & - & - \\
\hline 2 & S184_10:1 & Sylgard $184(10: 1)$ & - & - & - \\
\hline 3 & S186_5:1 & Sylgard $186(5: 1)$ & - & - & - \\
\hline 4 & S186_10:1 & Sylgard 186 (10:1) & - & - & - \\
\hline 5 & Eco 30 & Ecoflex 00-30 (1:1) & - & - & - \\
\hline 6 & Eco 50 & Ecoflex 00-50 (1:1) & - & - & - \\
\hline 7 & MIX Eco 30_S184_3:1 & Sylgard $184(10: 1)$ & 1 & Ecoflex 00-30 (1:1) & 3 \\
\hline 8 & MIX Eco 50_S184_3:1 & Sylgard 184 (10:1) & 1 & Ecoflex 00-50 (1:1) & 3 \\
\hline
\end{tabular}

*Mixing weight ratios for parts A and B (A:B) of the respective silicone kits (in brackets) 


\section{WILEY-VCH}

Table 2. Analysis of the gel fraction and TGA data of commercial silicone formulations.

\begin{tabular}{ccccc} 
Sample name & $\mathbf{w}_{\text {gel }}{ }^{*}$ & $\begin{array}{c}\text { Onset degradation } \\
{\left[{ }^{\circ} \mathrm{C}\right]^{* *}}\end{array}$ & $\begin{array}{c}\text { Residual weight at } 900^{\circ} \mathrm{C} \\
{[\%]^{* *}}\end{array}$ & $\begin{array}{c}\text { Amount of filler } \\
{[\%]^{* * *}}\end{array}$ \\
\hline S184_5:1 & 0.95 & 373 & 54.7 & 46 \\
\hline S184_10:1 & 0.95 & 380 & 68.3 & 68 \\
\hline S186_5:1 & 0.96 & 415 & 62.3 & 62 \\
\hline S186_10:1 & 0.96 & 428 & 30.2 & 30 \\
\hline Eco 30 & 0.44 & 308 & 31.9 & 31 \\
\hline Eco 50 & 0.52 & 301 & 40.3 & 40 \\
\hline MIX Eco 30_S184_3:1 & 0.58 & 297 & 44.6 & 44 \\
\hline
\end{tabular}

*Experimentally determined weight fraction of gel. $* *$ Values calculated as an average value of data from three TGA measurements. ***Amount of filler is estimated to be equal to the residual weight at $900^{\circ} \mathrm{C}$.

Table 3. SEC data of commercial silicone formulations. All samples show a bimodal distribution and thus a pair of number average and weight average molecular weights is given

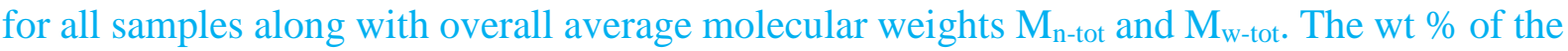
high molecular weight parts is stated as $\mathrm{W}_{\text {long, }}$, and overall wt \% of high molecular weight parts in the hard and soft formulations is stated as $\mathrm{W}_{\mathrm{f} \text { long chains. }}$

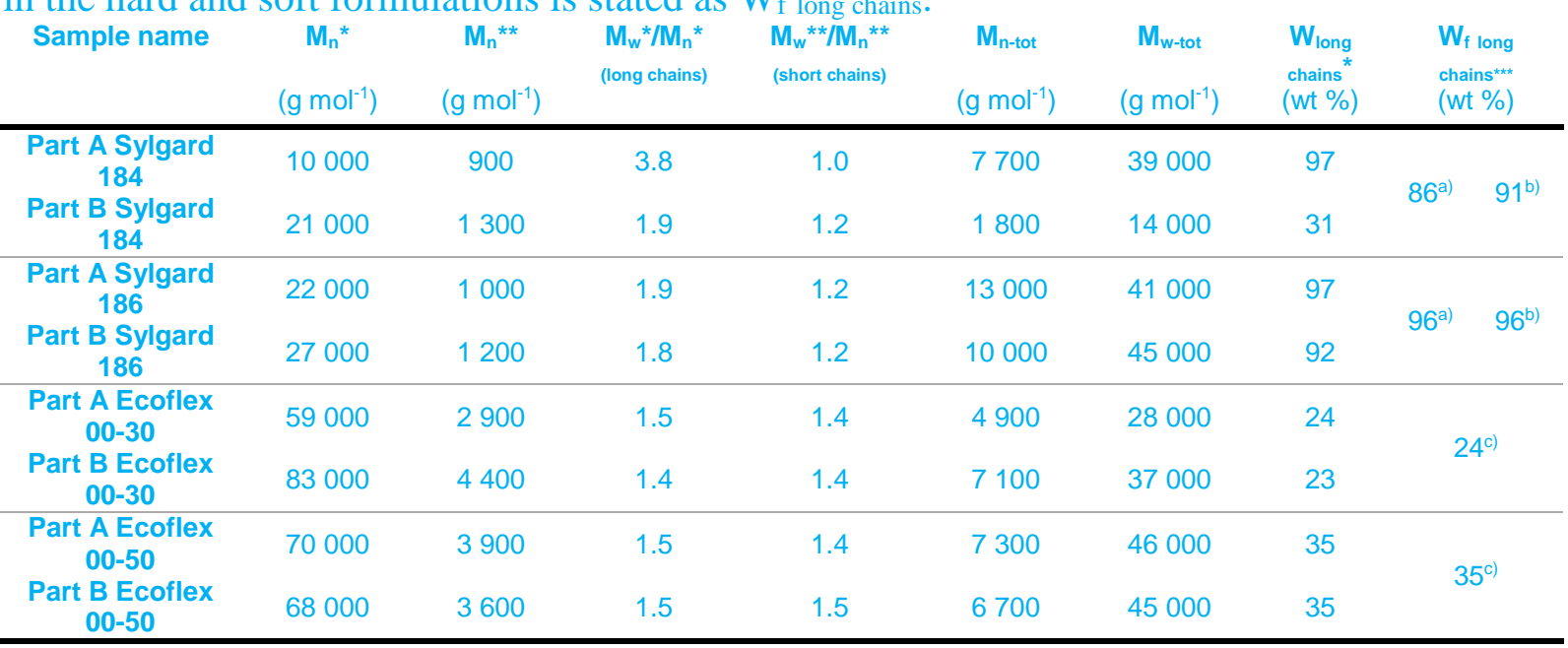

*Values determined from the first peak of size exclusion chromatography. $*$ * Values determined from the second peak of size exclusion chromatography. ***Values determined by accounting for mixing ratios of $\mathrm{A}$ and $\mathrm{B}$ parts in hard and soft formulations: a) ratio of $\mathrm{A}$ and $\mathrm{B}$ parts in hard formulations 5:1, b) ratio of $\mathrm{A}$ and $\mathrm{B}$ parts in hard formulations 10:1, and c) ratio of $\mathrm{A}$ and B parts in soft formulations 1:1. 


\section{WILEY-VCH}

Table 4. Summarized findings of different properties of commercial silicone formulations.

\begin{tabular}{|c|c|c|c|c|}
\hline Sample name & $\begin{array}{c}\text { Formulation } \\
\text { type* }\end{array}$ & $\begin{array}{l}\text { Network crosslinking } \\
\text { degree }^{\star \star}\end{array}$ & Amount of fillers ${ }^{\star \star \star}$ & $\begin{array}{l}\text { Molecular } \\
\text { weight }^{\star \star \star \star}\end{array}$ \\
\hline S184_5:1 & Hard & High & High (mostly resins) & Low \\
\hline S184_10:1 & Hard & High & High (mostly resins) & Low \\
\hline S186_5:1 & Hard & High & High & Medium \\
\hline S186_10:1 & Hard & High & High & Medium \\
\hline Eco 30 & Soft & Low & Low & High \\
\hline Eco 50 & Soft & Medium & Low & High \\
\hline MIX Eco 30_S184_3:1 & Blend & Medium & Medium & - \\
\hline MIX Eco 50_S184_3:1 & Blend & Medium & Medium & - \\
\hline \multicolumn{5}{|c|}{$\begin{array}{l}\text { *Evaluation based on physical and mechanical characteristics of different formulations } \\
\text { **Evaluation made by comparing values of sample films' gel fractions. *** Evaluation mad } \\
\text { by comparing filler content in different formulations and by accounting viscosities an } \\
\text { transparencies of different formulations. ****Evaluation made by comparing molecula } \\
\text { weights of long and short polymer chains in A and B parts of different formulations. }\end{array}$} \\
\hline
\end{tabular}

Table 5. Ultimate mechanical properties, $\varepsilon_{\mathrm{T}-\mathrm{STD}}$ and $\varepsilon_{\max }$ of different silicone elastomers

\begin{tabular}{cccccc} 
Sample name & $\mathrm{Y}[\mathrm{MPa}]$ & $\boldsymbol{\varepsilon}_{\mathrm{T}}[\%]$ & $\boldsymbol{\varepsilon}_{\mathrm{T}-\mathrm{STD}}[\%]$ & $\boldsymbol{\sigma}_{\mathrm{T}}[\mathrm{MPa}]$ & $\boldsymbol{\varepsilon}_{\max }[\%]^{\star}$ \\
\hline S184_5:1 & 2.57 & 113 & 10.0 & 7.51 & 100 \\
\hline S184_10:1 & 2.26 & 132 & 11.2 & 7.35 & 120 \\
\hline S186_5:1 & 1.33 & 380 & 8.30 & 5.71 & 340 \\
\hline S186_10:1 & 1.01 & 434 & 47.3 & 5.96 & 340 \\
\hline Eco 30 & 0.16 & 730 & 49.9 & 1.45 & 680 \\
\hline Eco 50 & 0.23 & 749 & 14.5 & 2.53 & 680 \\
\hline MIX Eco 30_S184_3:1 & 0.41 & 254 & 33.5 & 1.86 & 180 \\
\hline MIX Eco 50_S184_3:1 & 0.62 & 252 & 9.39 & 2.00 & 240 \\
\hline
\end{tabular}

*Determined maximum tensile strain value up to which different sample formulations can be stretched in the cyclic tests, without breaking. 


\section{WILEY-VCH}

Silicone elastomers used for soft and stretchable robotics have to maintain their chemical, thermal and mechanical properties during specified lifetime of the device. Mullins effect is one of the effects, that has potential to alter elastomer properties, and in that way significantly affect the work of the soft and stretchable device. Mullins effect is investigated in commercial silicone elastomer formulations which are commonly used for soft robotics.

\section{Mullins effect}

Sara Krpovic, Kim Dam-Johansen and Anne Ladegaard Skov*

\section{Importance of Mullins effect in commercial silicone elastomer formulations for soft robotics}
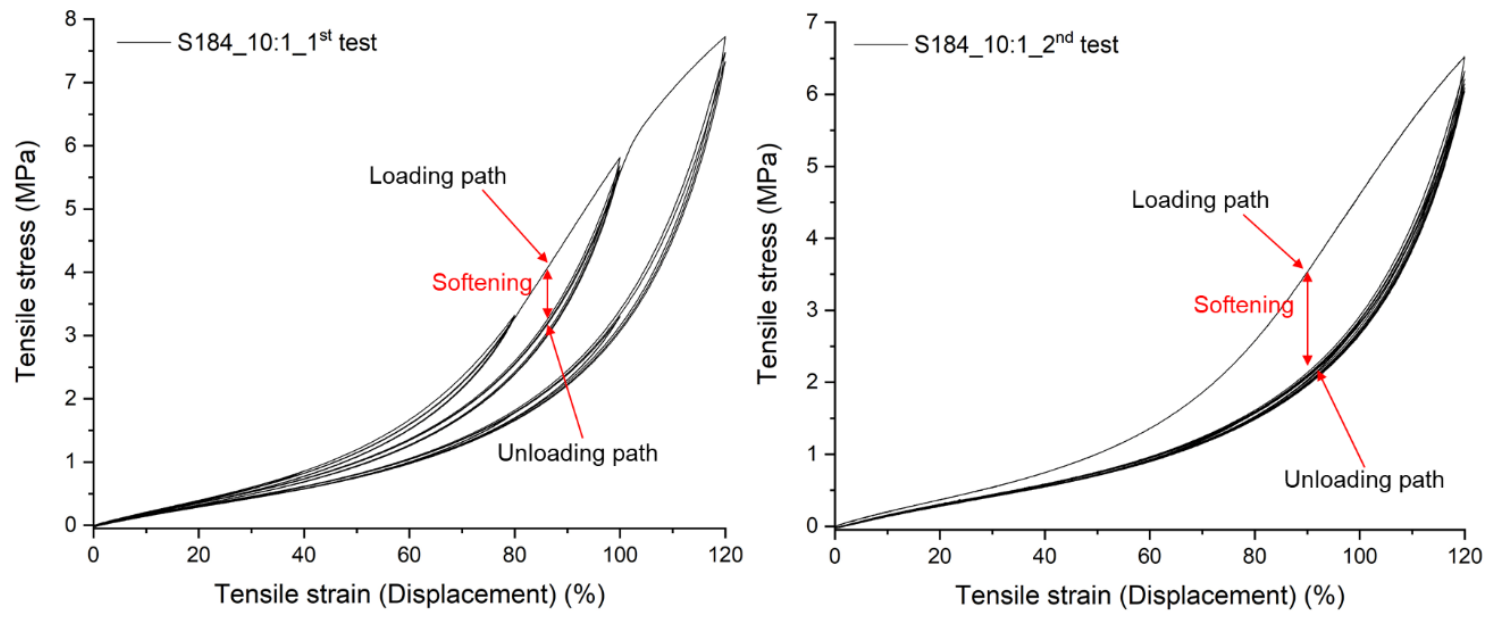


\section{WILEY-VCH}

Copyright WILEY-VCH Verlag GmbH \& Co. KGaA, 69469 Weinheim, Germany, 2018.

\section{Supporting Information}

\section{Importance of Mullins effect in commercial silicone elastomer formulations for soft robotics}

Sara Krpovic, Kim Dam-Johansen and Anne Ladegaard Skov*

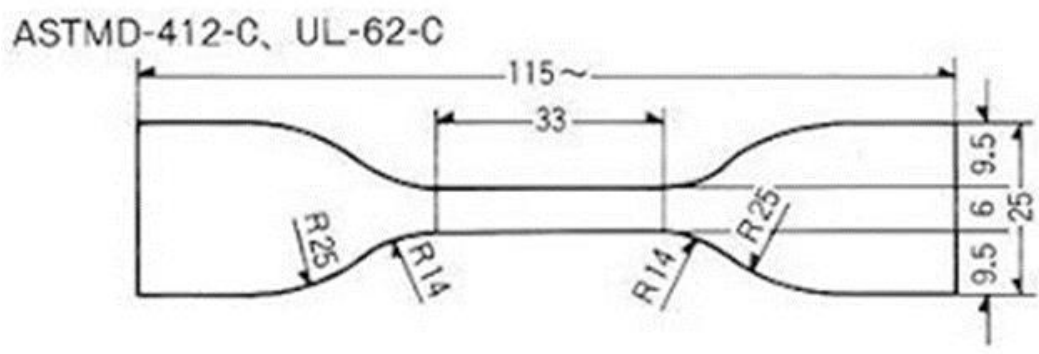

Figure 1S. Schematic illustration of a "Dog bone" shape ASTM D412 standard. Size is given in $\mathrm{mm}$.

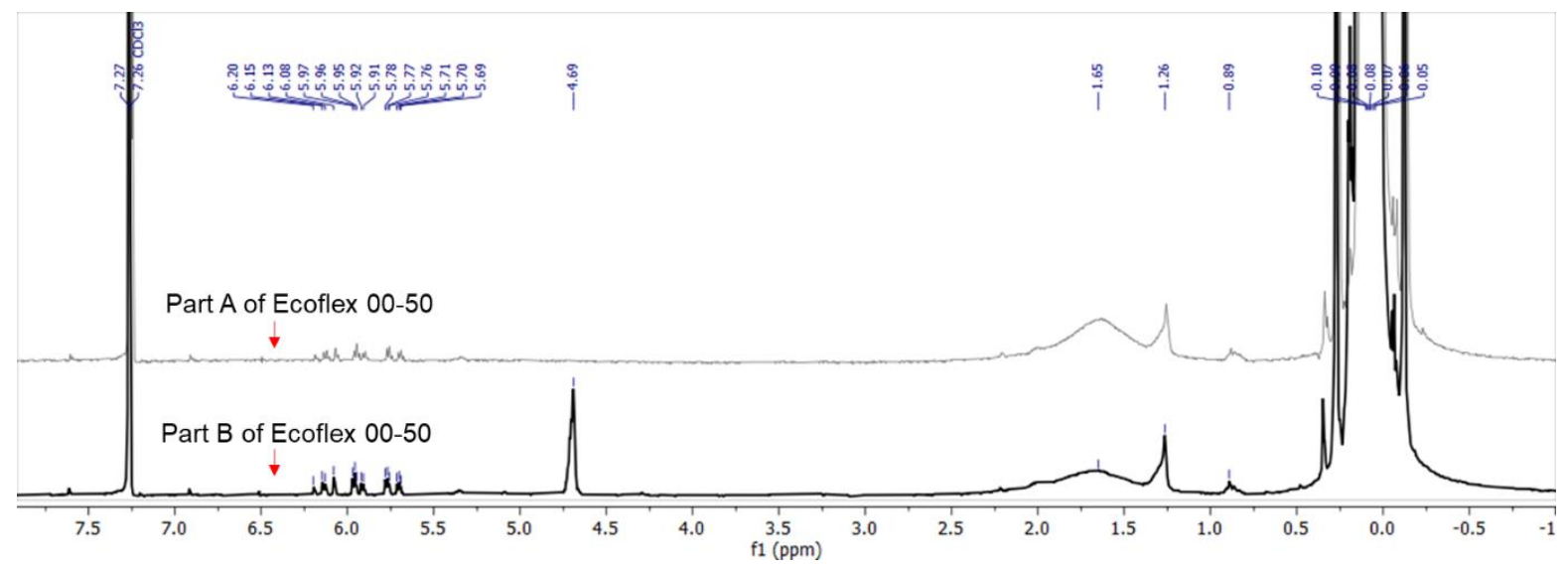

Figure 2S. 1H NMR spectra of extracts of part A (grey line) and part B (black line) of Ecoflex $00-50$. 
WILEY-VCH

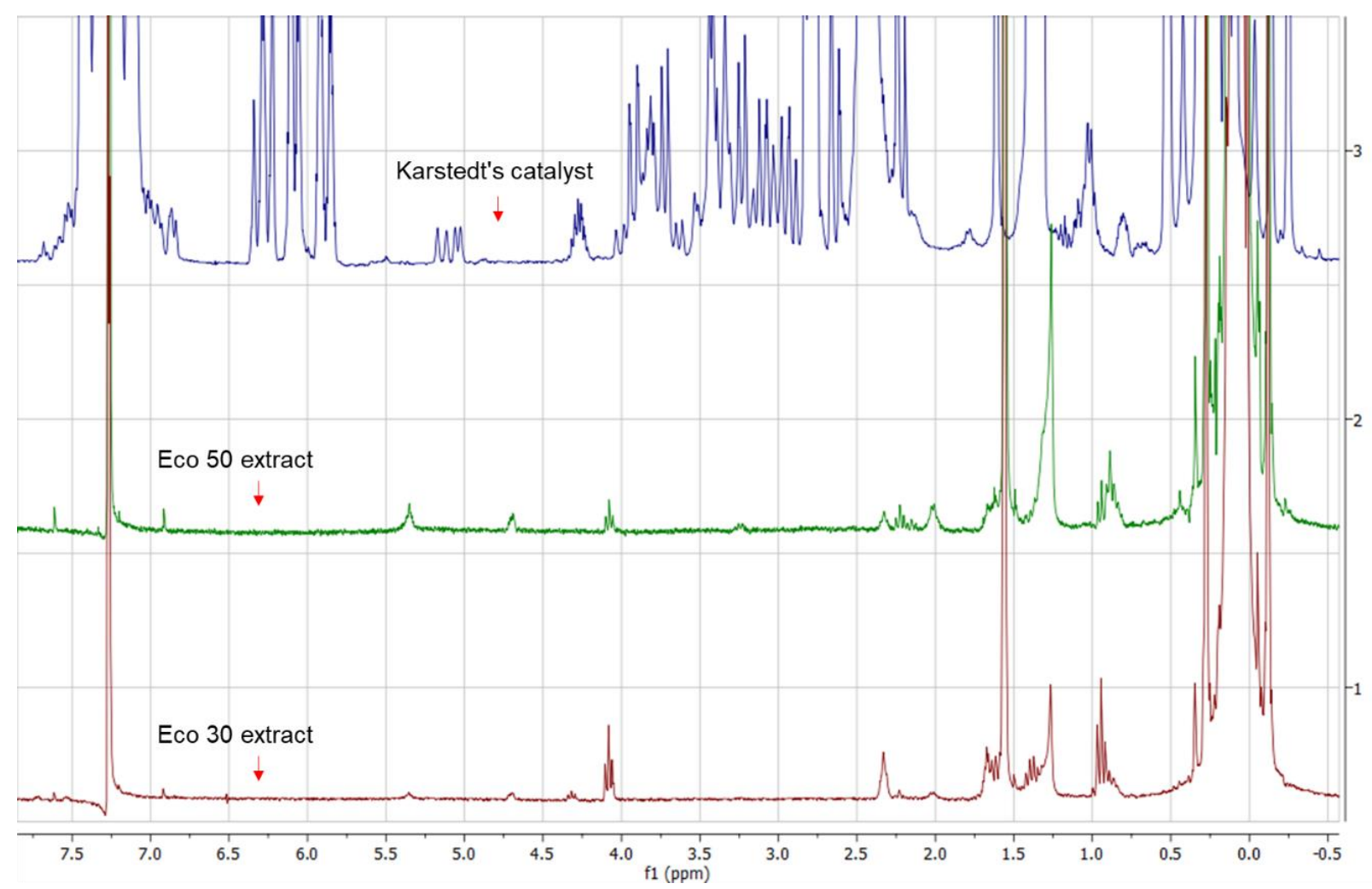

Figure 3S. 1H NMR spectra of extracts of Eco 30 (red) and Eco 50 (green), and Karstedt's catalyst in xylene (blue).

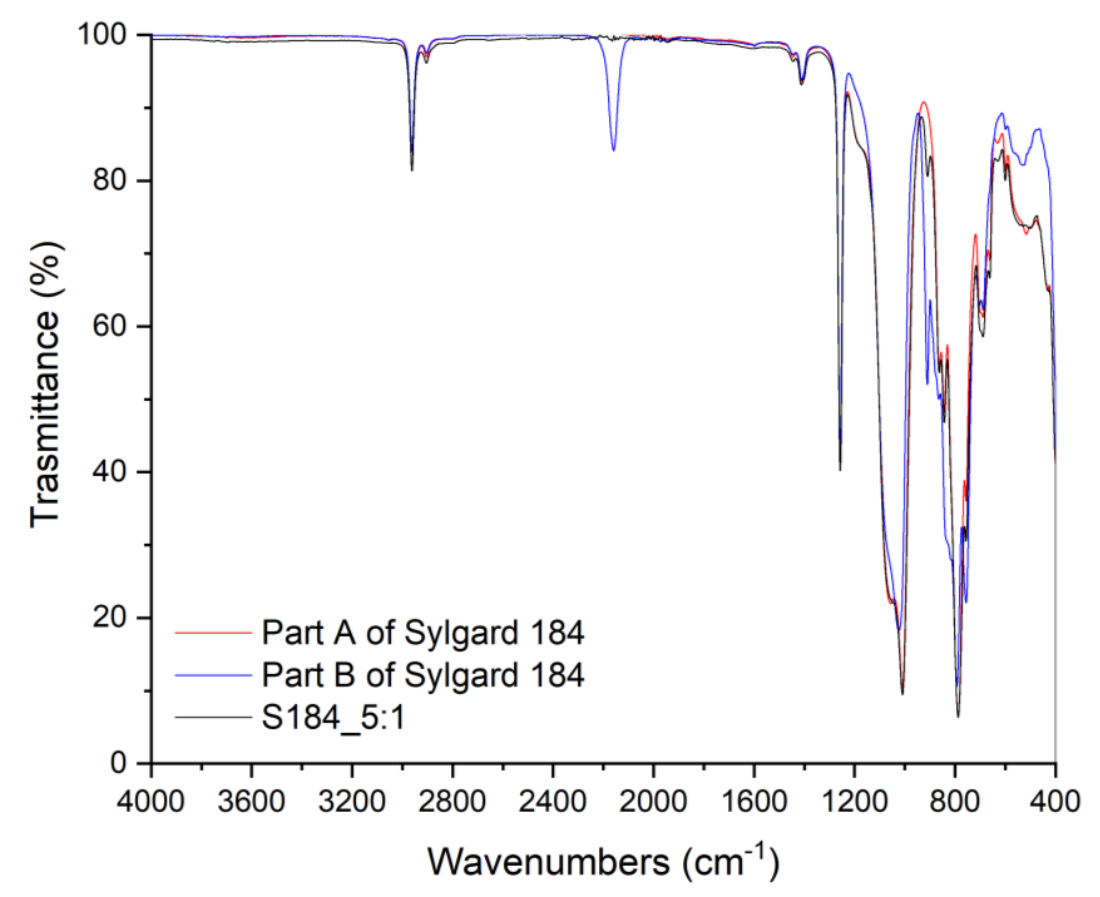

Figure 4S. FTIR spectra of part A (red) and part B (blue) of Sylgard 184 formulation, and S184_5:1 sample (black). 


\section{WILEY-VCH}

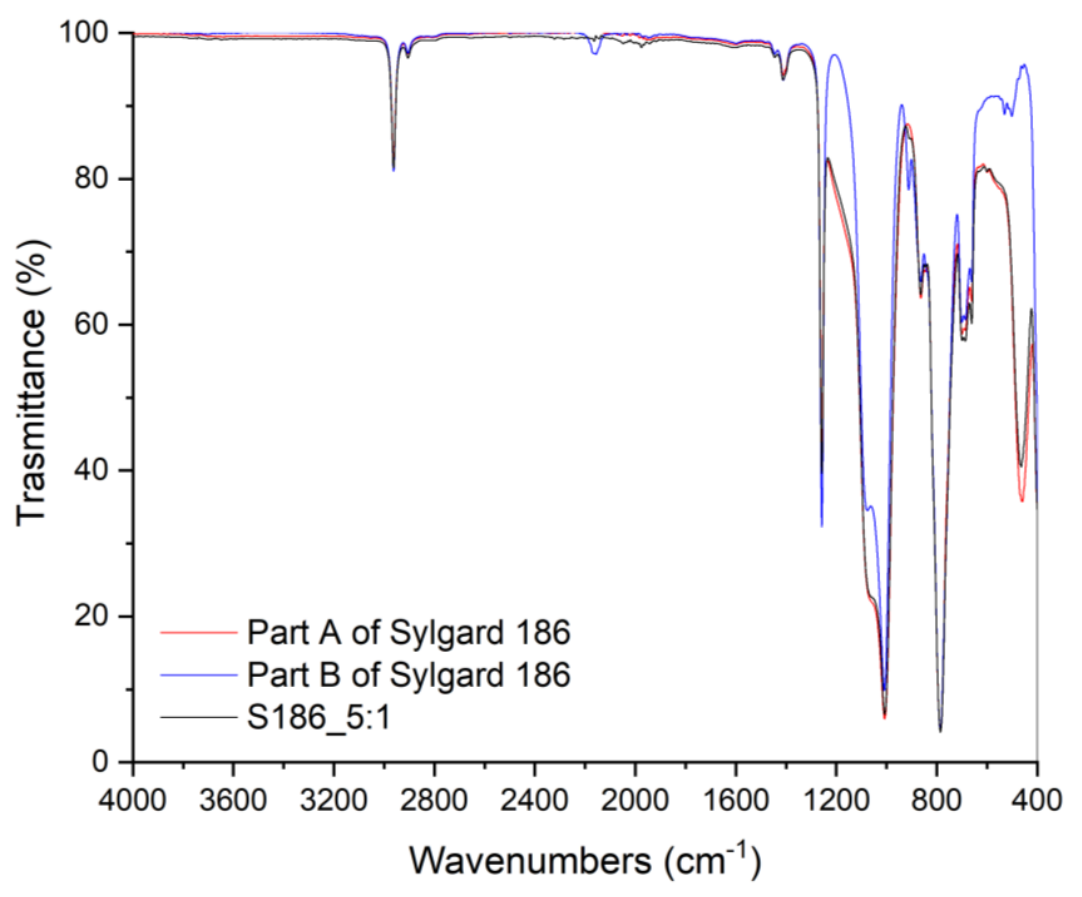

Figure 5S. FTIR spectra of part A (red) and part B (blue) of Sylgard 186 formulation, and S186_5:1 sample (black).

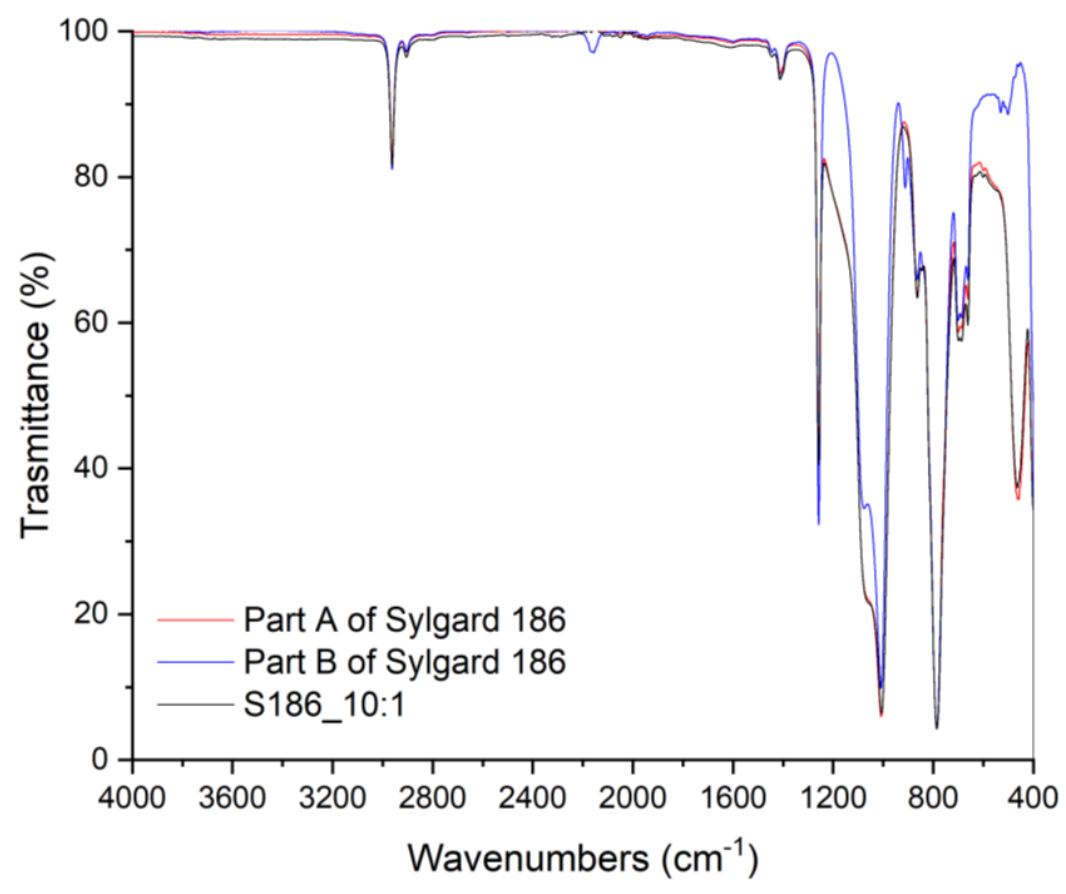

Figure 6S. FTIR spectra of part A (red) and part B (blue) of Sylgard 186 formulation, and S186_10:1 sample (black). 


\section{WILEY-VCH}

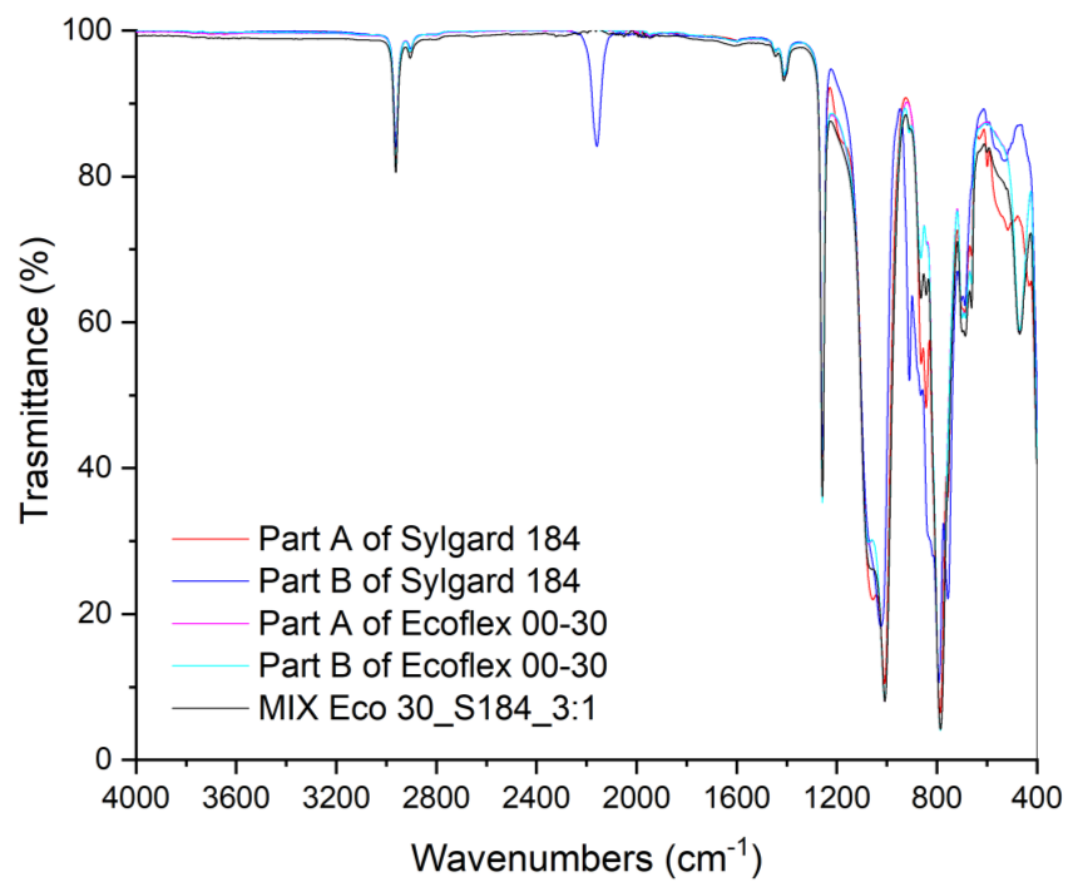

Figure 7S. FTIR spectra of part A (red) and part B (blue) of Sylgard 184 formulation, part A (pink) and part B (turquoise) of Ecoflex 00-30 formulation and MIX Eco 30_S184_3:1 sample (black).

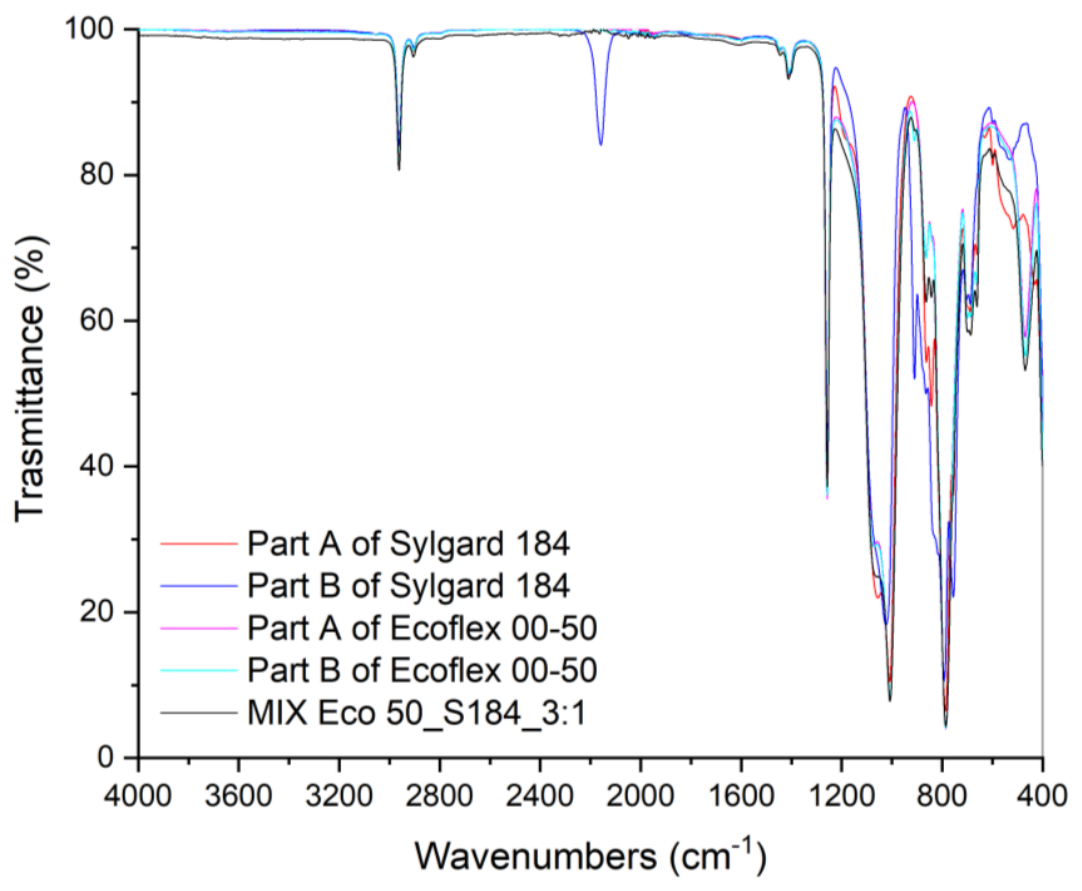

Figure 8S. FTIR spectra of part A (red) and part B (blue) of Sylgard 184 formulation, part A (pink) and part B (turquoise) of Ecoflex 00-50 formulation and MIX Eco 50_S184_3:1 sample (black). 


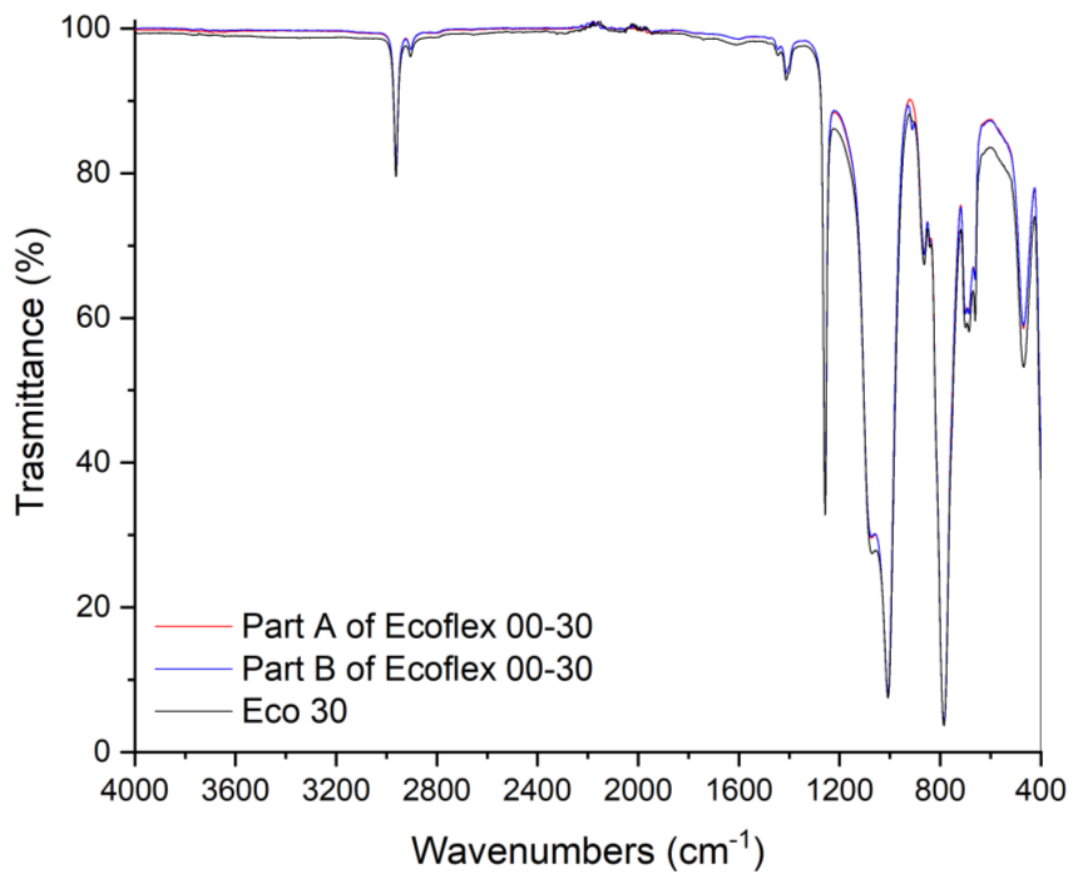

Figure 9S. FTIR spectra of part A (red) and part B (blue) of Ecoflex 00-30 formulation, and Eco 30 sample (black).

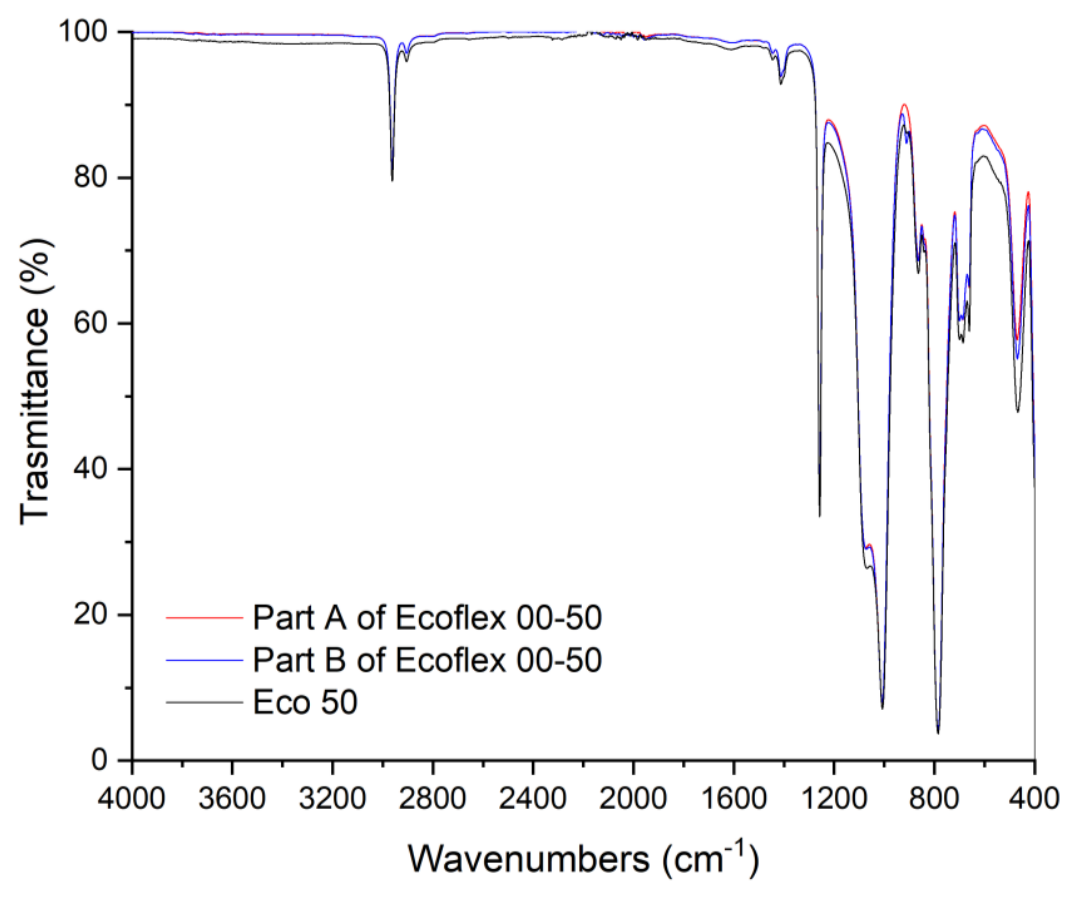

Figure 10S. FTIR spectra of part A (red) and part B (blue) of Ecoflex 00-30 formulation, and Eco 50 sample (black). 


\section{WILEY-VCH}

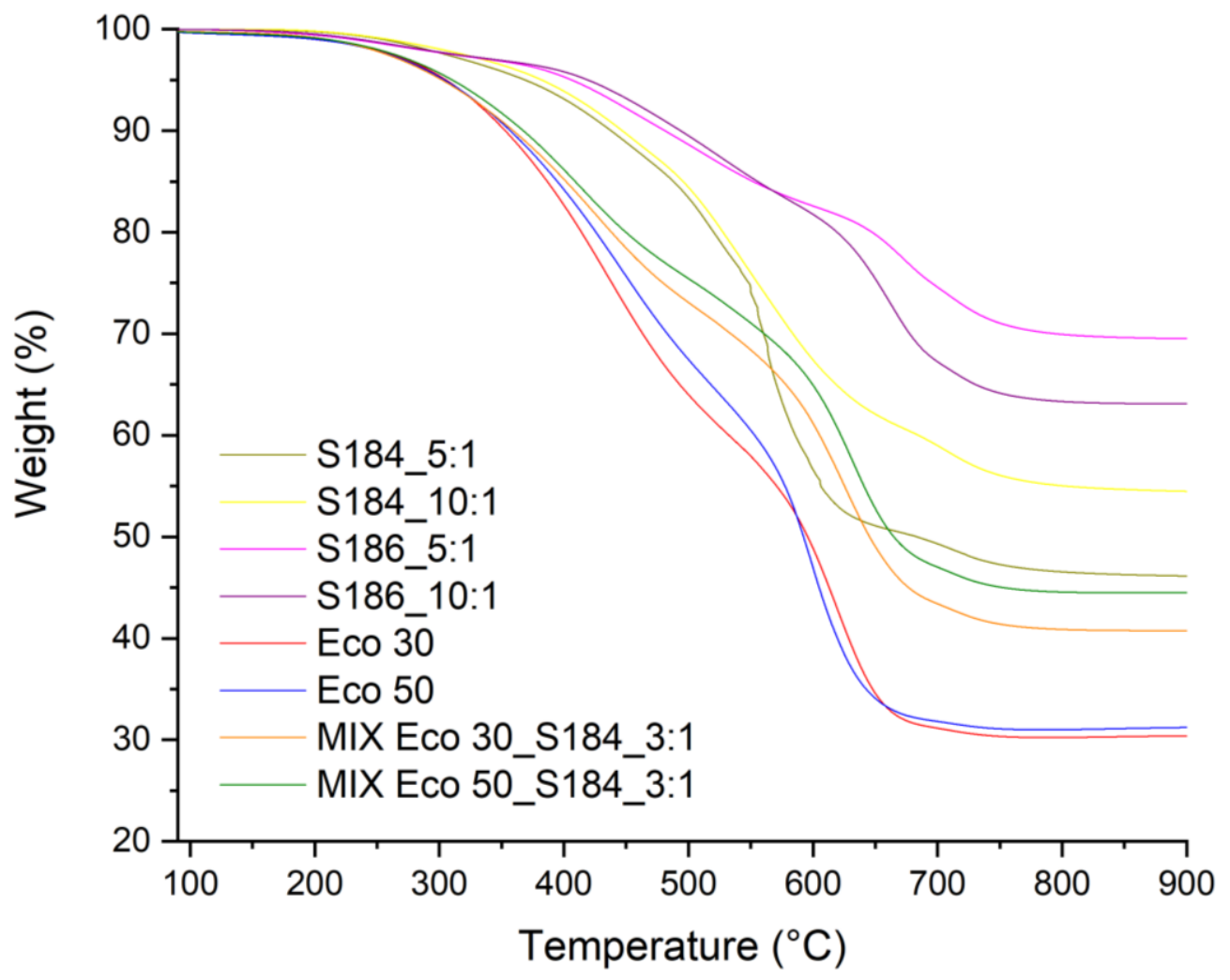

Figure 11S. TGA curves of different silicone formulations.

a)

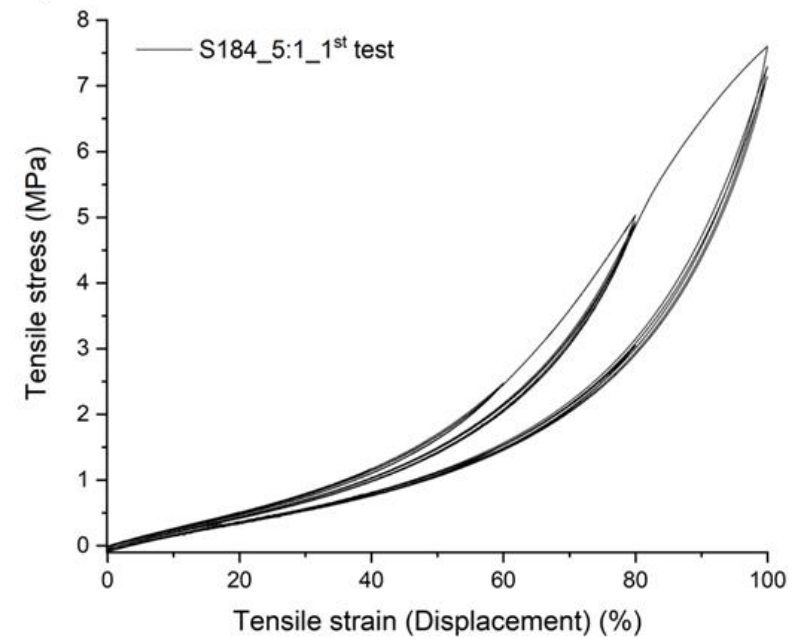

b)

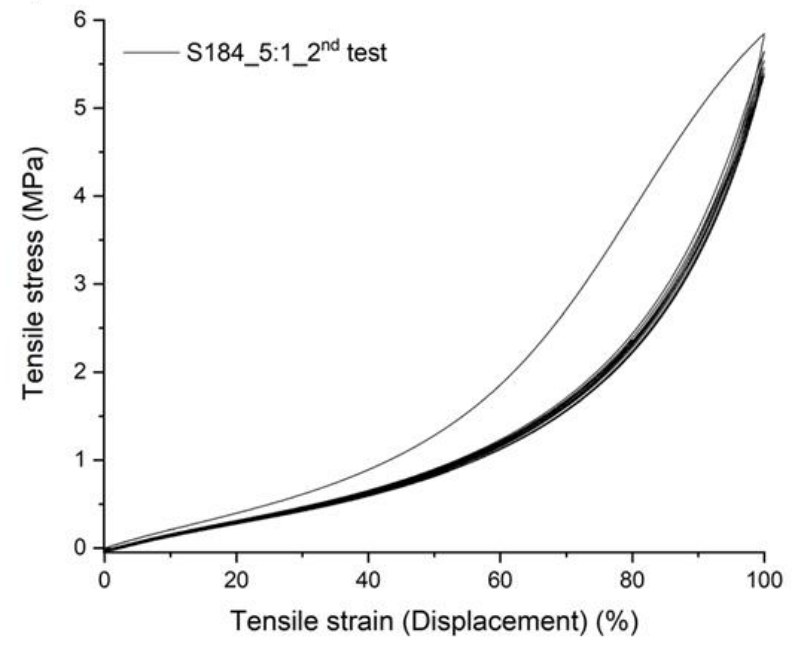

Figure 12S. Stress-strain response of S184_5:1 submitted to a) $1^{\text {st }}$ test, in which cycle strain is gradually increased, and b) $2^{\text {nd }}$ test, in which sample is subjected to maximum strain first. Tests were performed with $2 \mathrm{~mm} / \mathrm{s}$ strain rate and at room temperature $\left(23^{\circ} \mathrm{C}\right)$. 


\section{WILEY-VCH}

a)

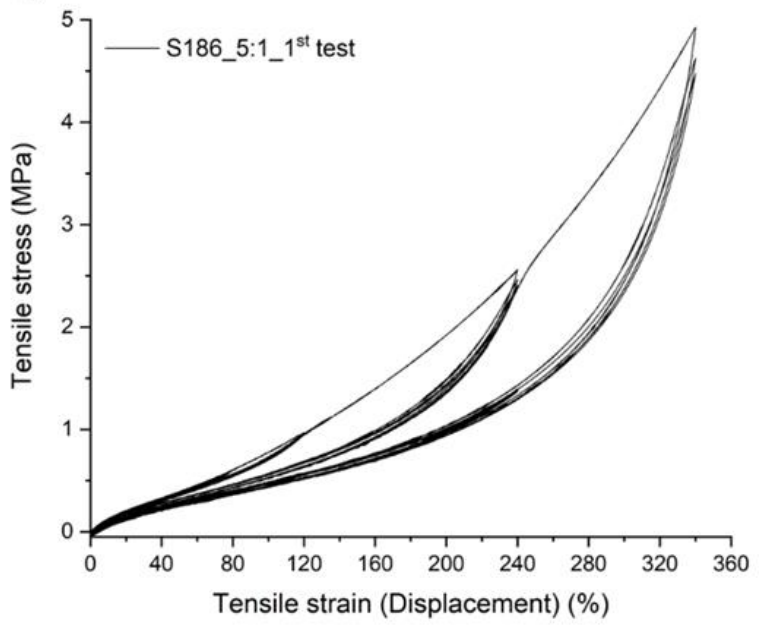

b)

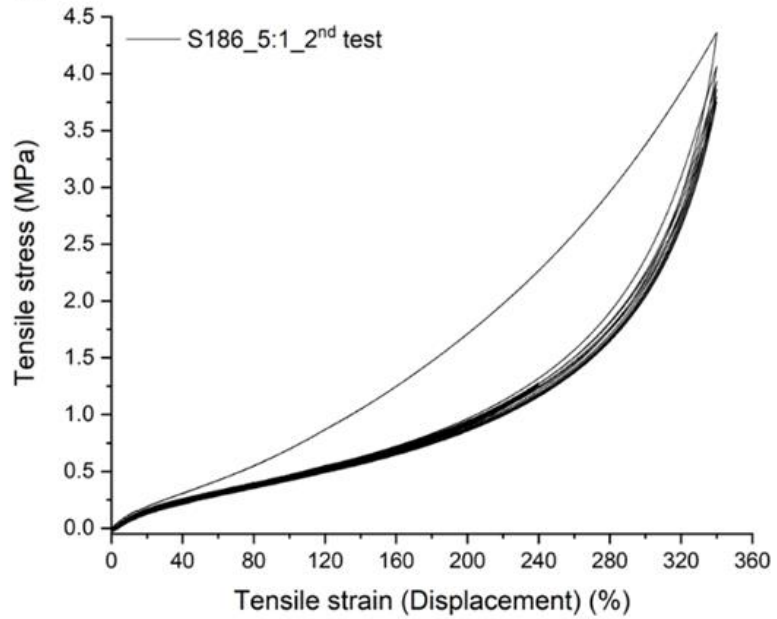

Figure 13S. Stress-strain response of S186_5:1 submitted to a) $1^{\text {st }}$ test, in which cycle strain is gradually increased, and b) $2^{\text {nd }}$ test, in which sample is subjected to maximum strain first. Tests were performed with $2 \mathrm{~mm} / \mathrm{s}$ strain rate and at room temperature $\left(23^{\circ} \mathrm{C}\right)$.

a)

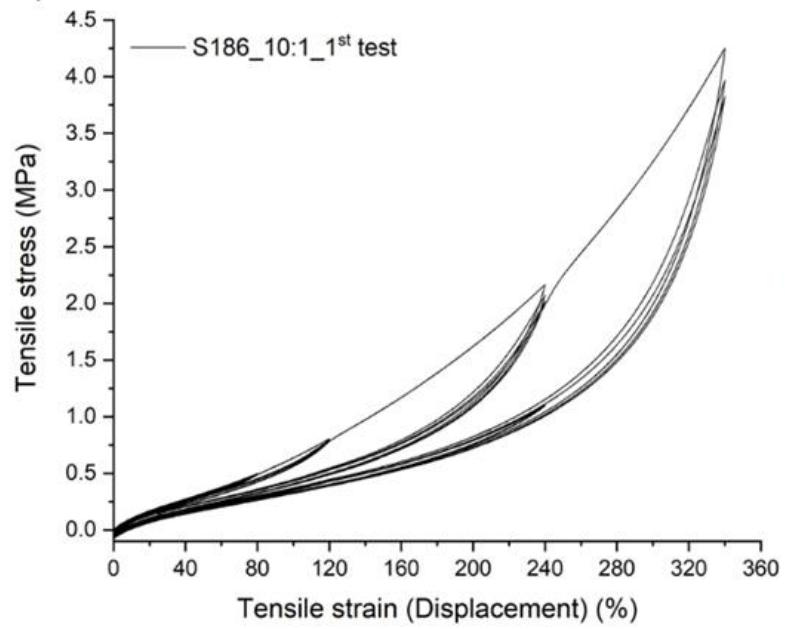

b)

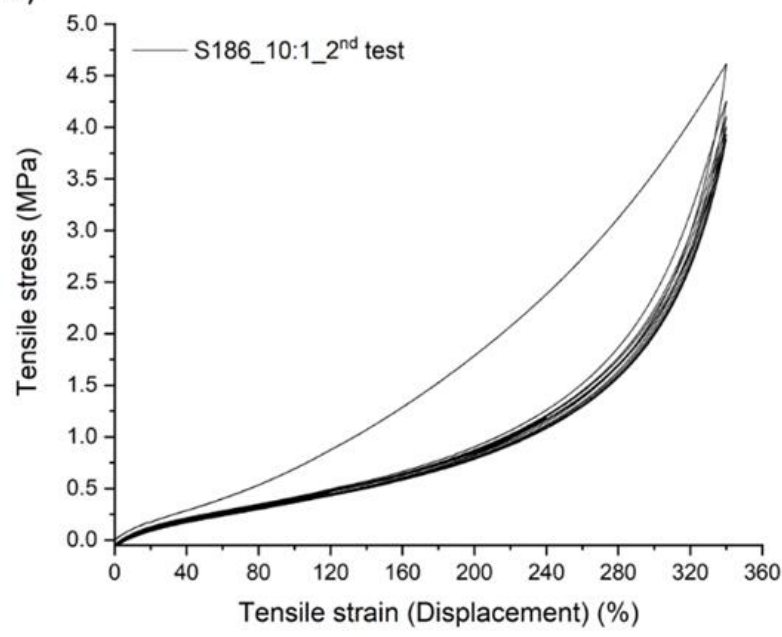

Figure 14S. Stress-strain response of S186_10:1 submitted to a) $1^{\text {st }}$ test, in which cycle strain is gradually increased, and b) $2^{\text {nd }}$ test, in which sample is subjected to maximum strain first. Tests were performed with $2 \mathrm{~mm} / \mathrm{s}$ strain rate and at room temperature $\left(23^{\circ} \mathrm{C}\right)$. 
a)

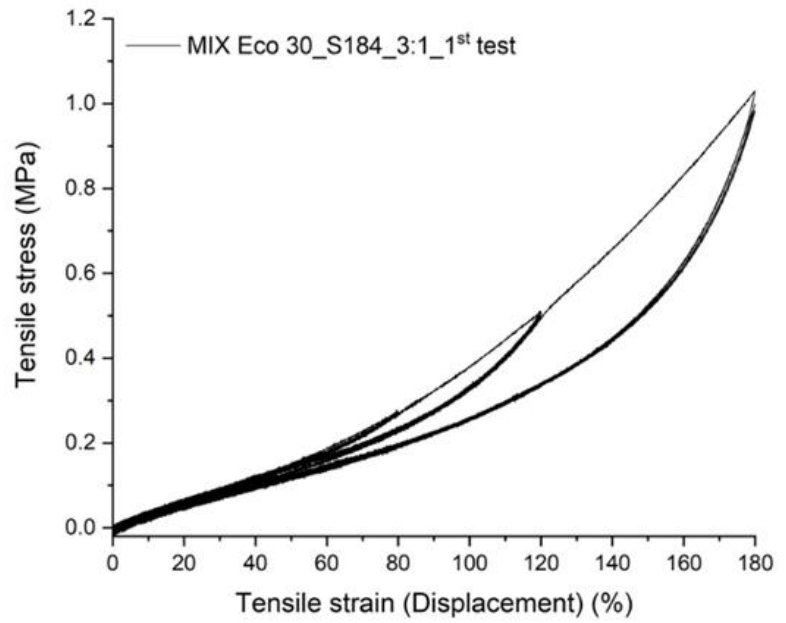

b)

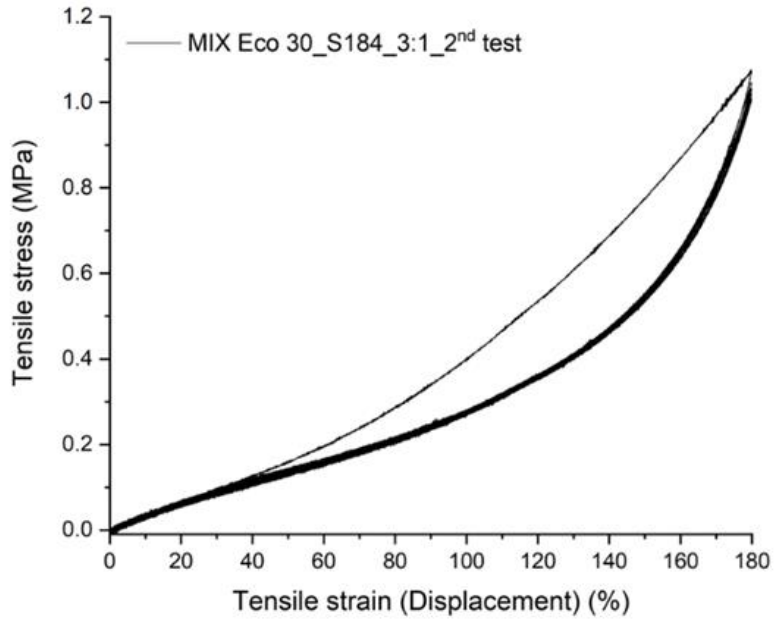

Figure 15S. Stress-strain response of MIX Eco 30_S184_3:1 submitted to a) $1^{\text {st }}$ test, in which cycle strain is gradually increased, and b) $2^{\text {nd }}$ test, in which sample is subjected to maximum strain first. Tests were performed with $2 \mathrm{~mm} / \mathrm{s}$ strain rate and at room temperature $\left(23^{\circ} \mathrm{C}\right)$.

a)

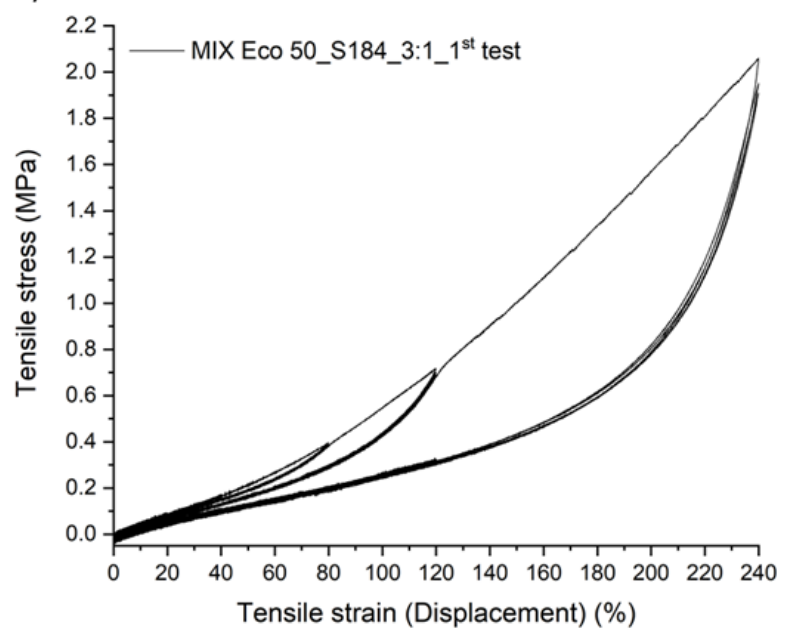

b)

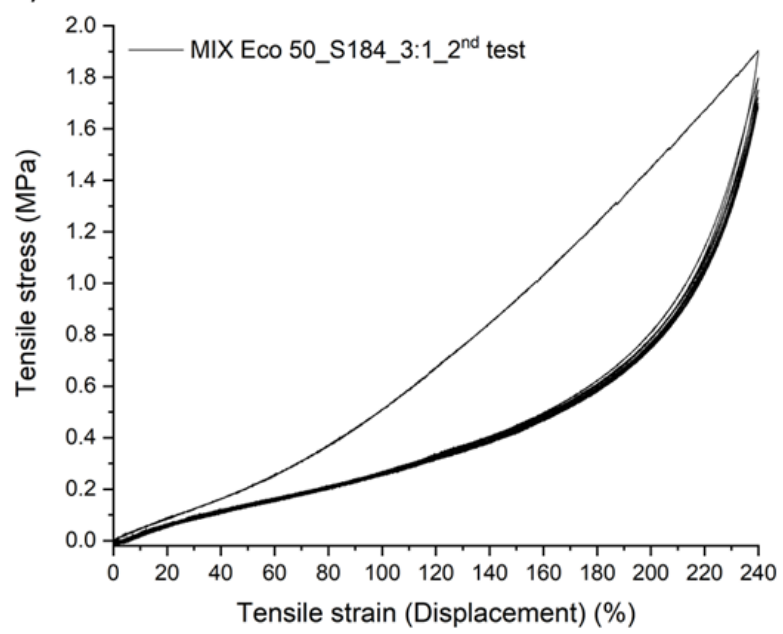

Figure 16S. Stress-strain response of MIX Eco 50_S184_3:1 submitted to a) $1^{\text {st }}$ test, in which cycle strain is gradually increased, and b) $2^{\text {nd }}$ test, in which sample is subjected to maximum strain first. Tests were performed with $2 \mathrm{~mm} / \mathrm{s}$ strain rate and at room temperature $\left(23^{\circ} \mathrm{C}\right)$. 


\section{WILEY-VCH}

a)

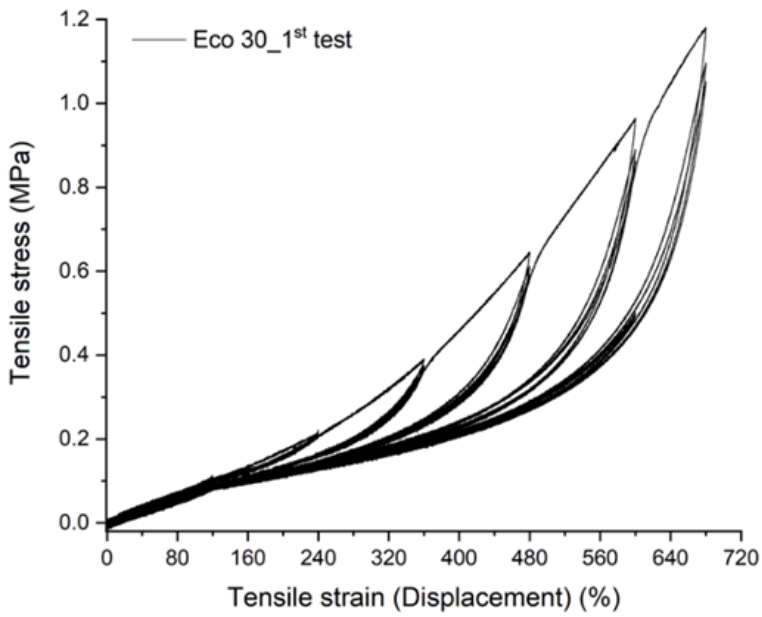

b)

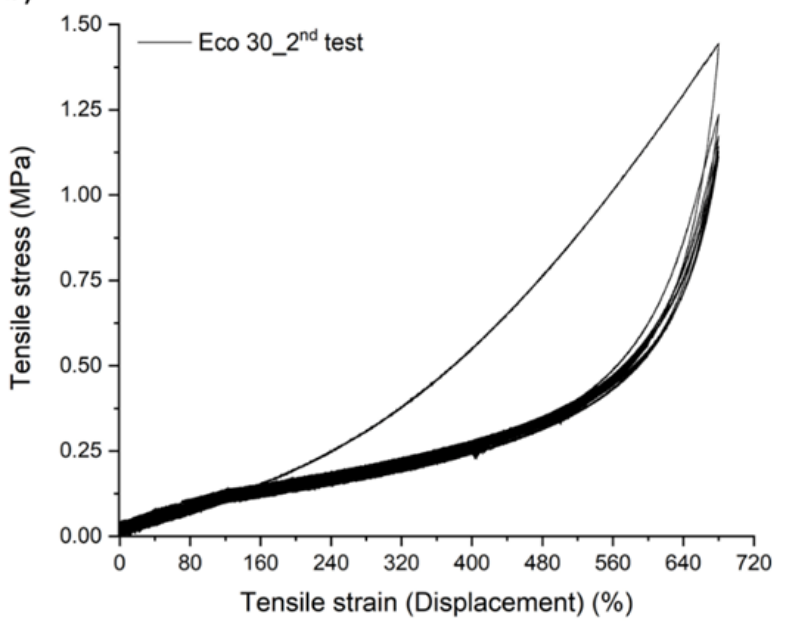

Figure 17S. Stress-strain response of Eco 30 submitted to a) $1^{\text {st }}$ test, in which cycle strain is gradually increased, and b) $2^{\text {nd }}$ test, in which sample is subjected to maximum strain first. Tests were performed with $2 \mathrm{~mm} / \mathrm{s}$ strain rate and at room temperature $\left(23^{\circ} \mathrm{C}\right)$.

a)

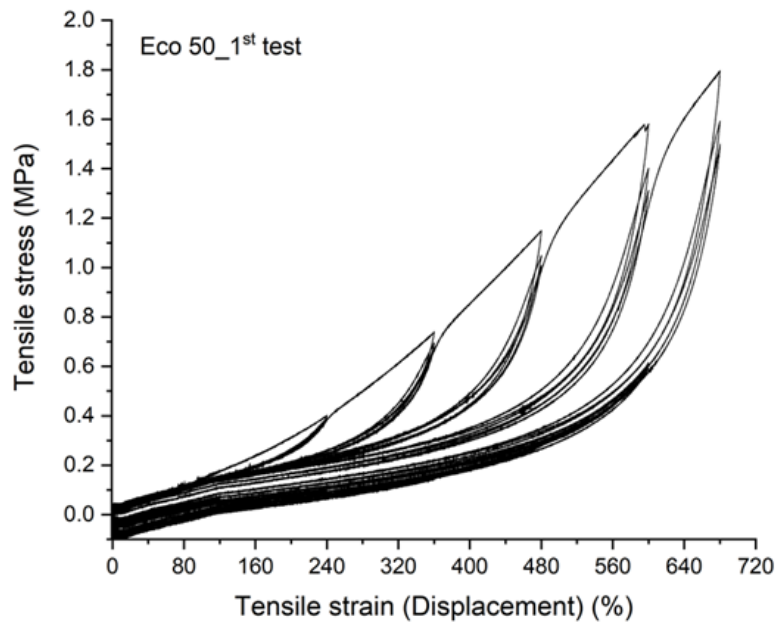

b)

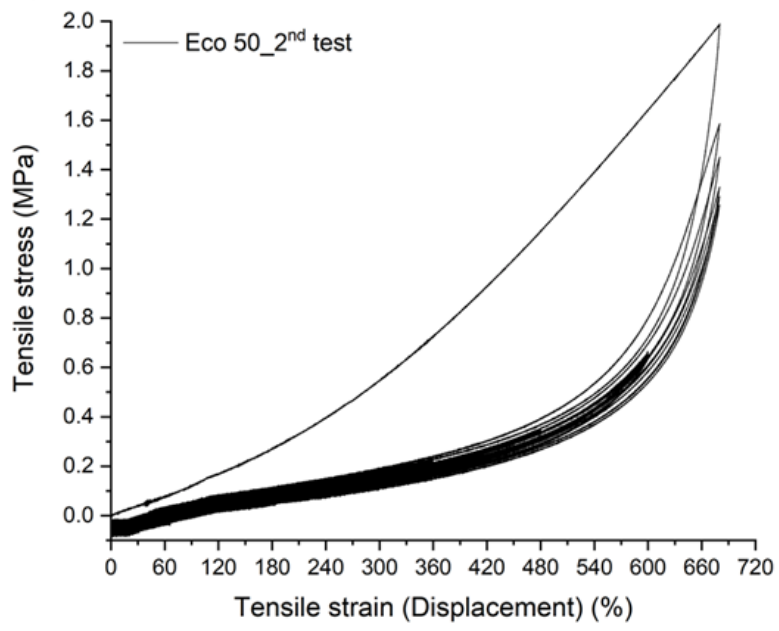

Figure 18S. Stress-strain response of Eco 50 submitted to a) $1^{\text {st }}$ test, in which cycle strain is gradually increased, and b) $2^{\text {nd }}$ test, in which sample is subjected to maximum strain first. Tests were performed with $2 \mathrm{~mm} / \mathrm{s}$ strain rate and at room temperature $\left(23^{\circ} \mathrm{C}\right)$. 


\section{WILEY-VCH}

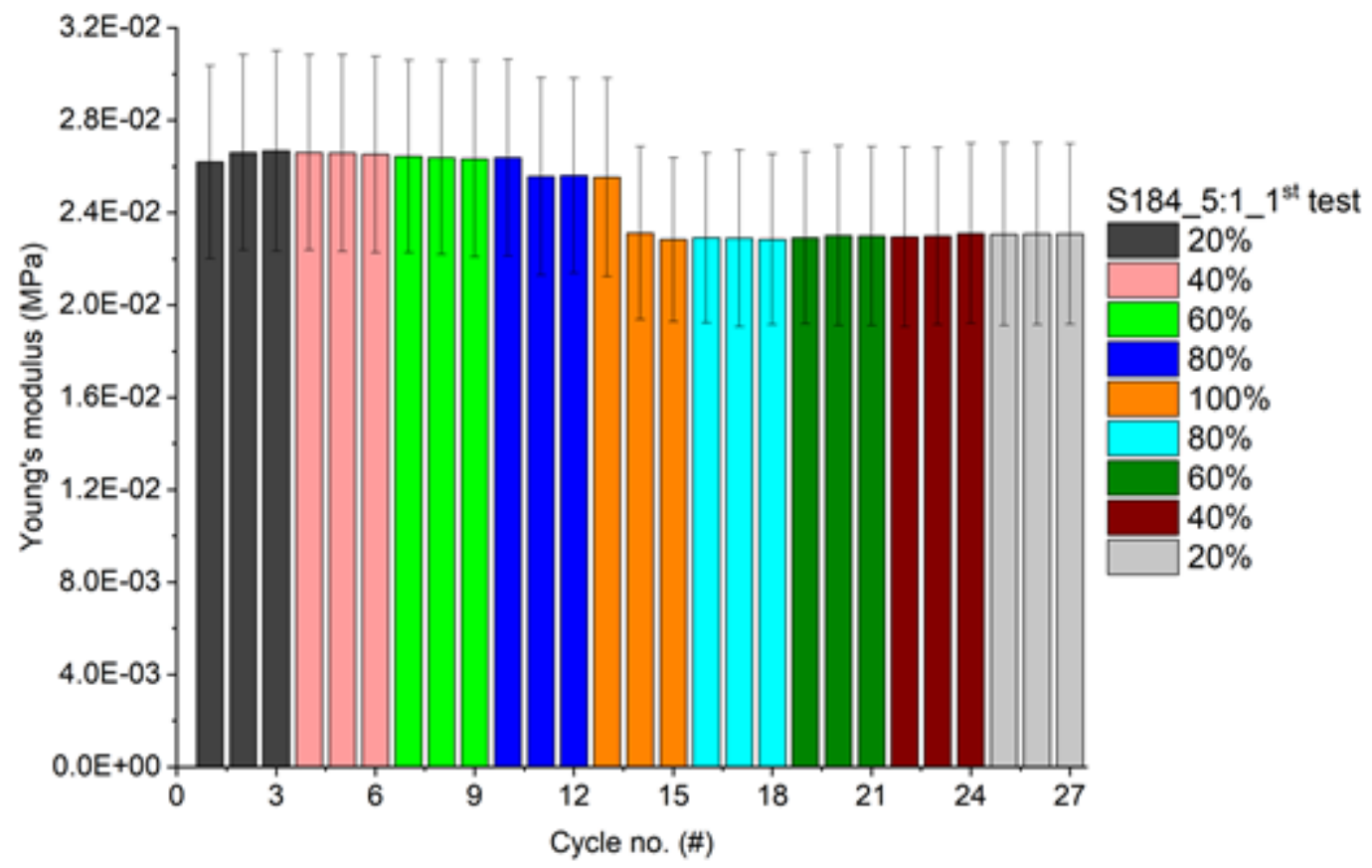

Figure 19S. Young's modulus (at 10\% strain) as a function of cycle number for different strains of S184_5:1 subjected to a $1^{\text {st }}$ test. After cycles 13-15, the strain path was reversed.

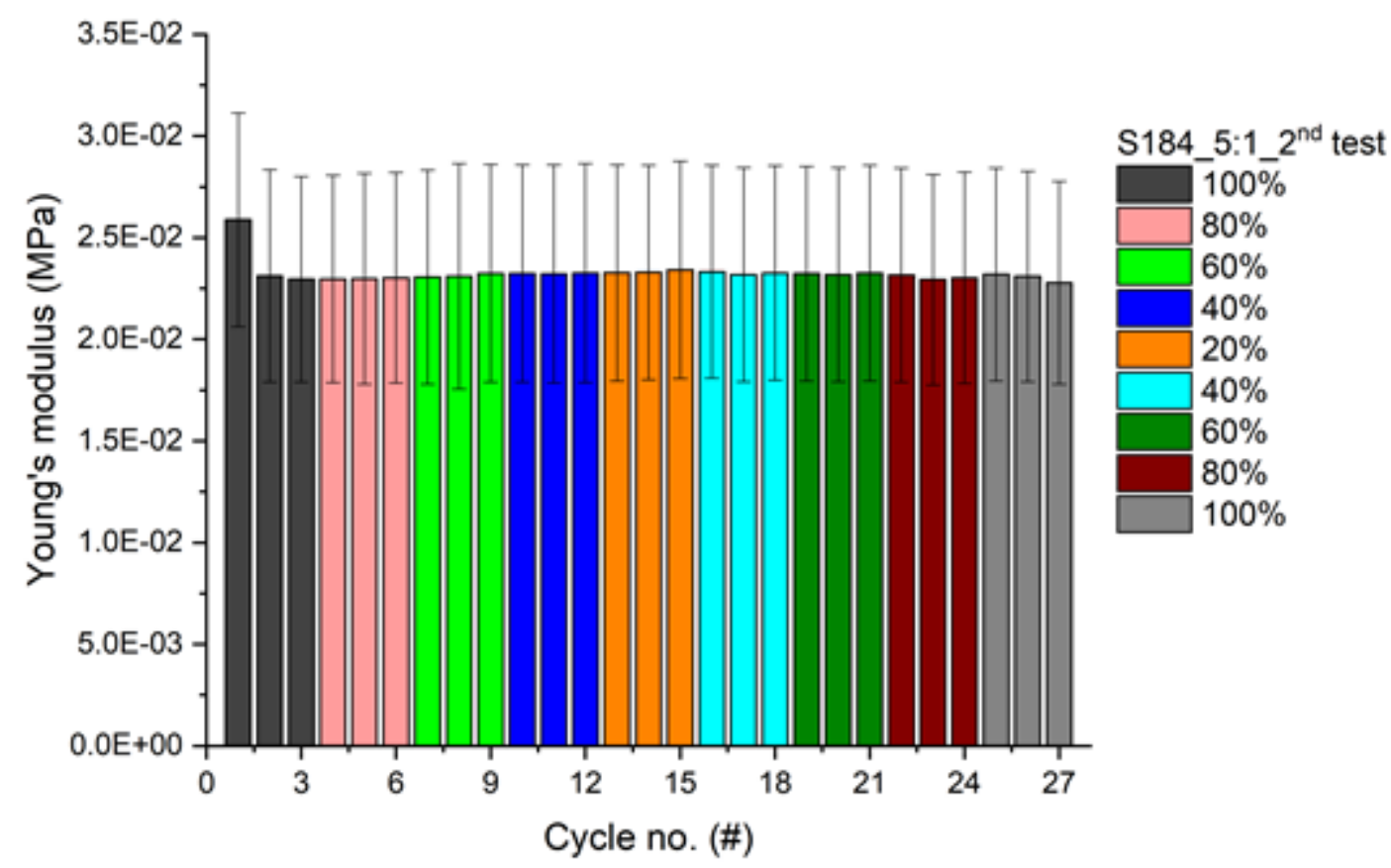

Figure 20S. Young's modulus (at 10\% strain) as a function of cycle number for different strains of S184_5:1 subjected to a $2^{\text {nd }}$ test. After cycles 13-15, the strain path was reversed. 


\section{WILEY-VCH}

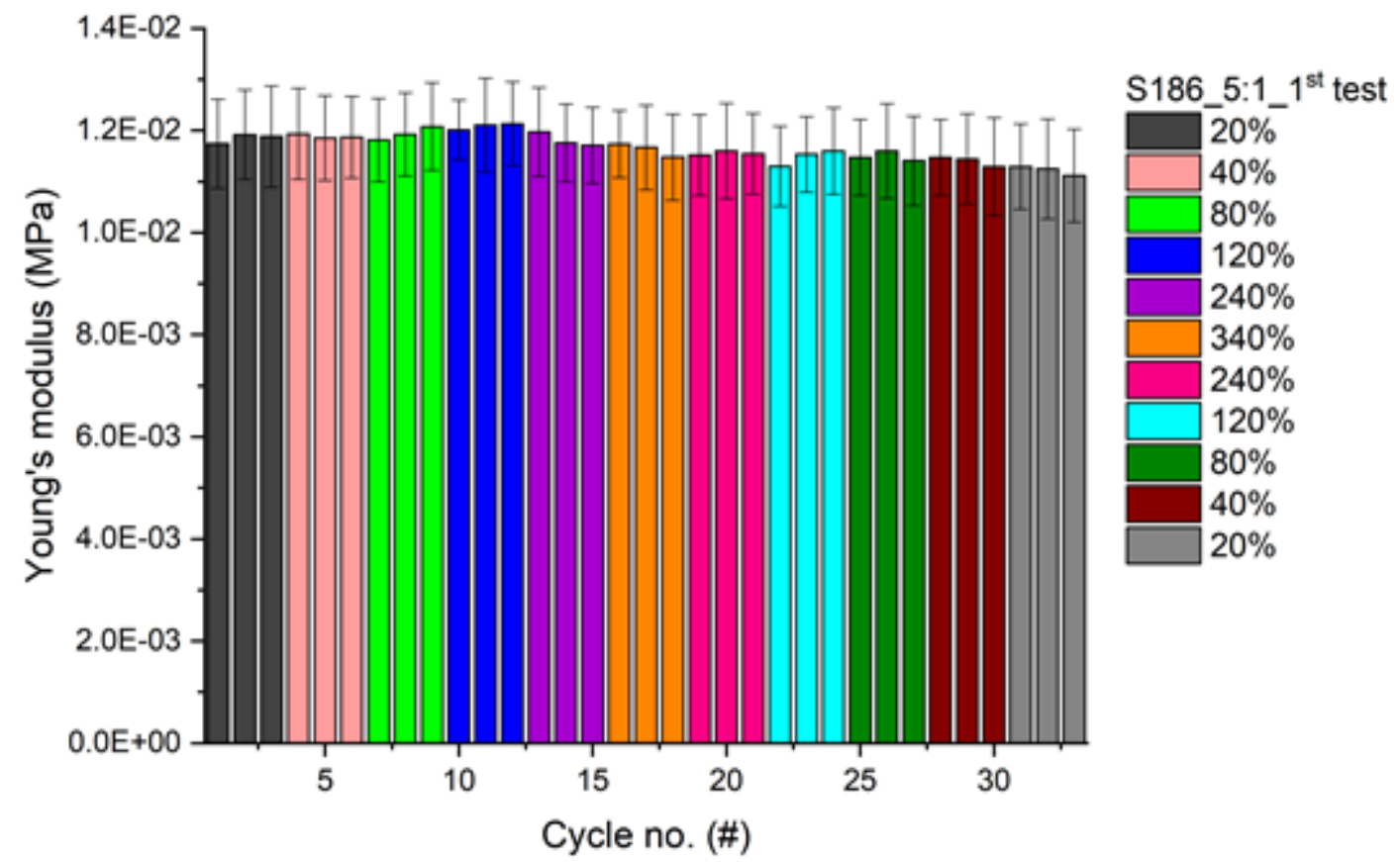

Figure 21S. Young's modulus (at 10\% strain) as a function of cycle number for different strains of S186_5:1 subjected to a $1^{\text {st }}$ test. After cycles 16-18, the strain path was reversed.

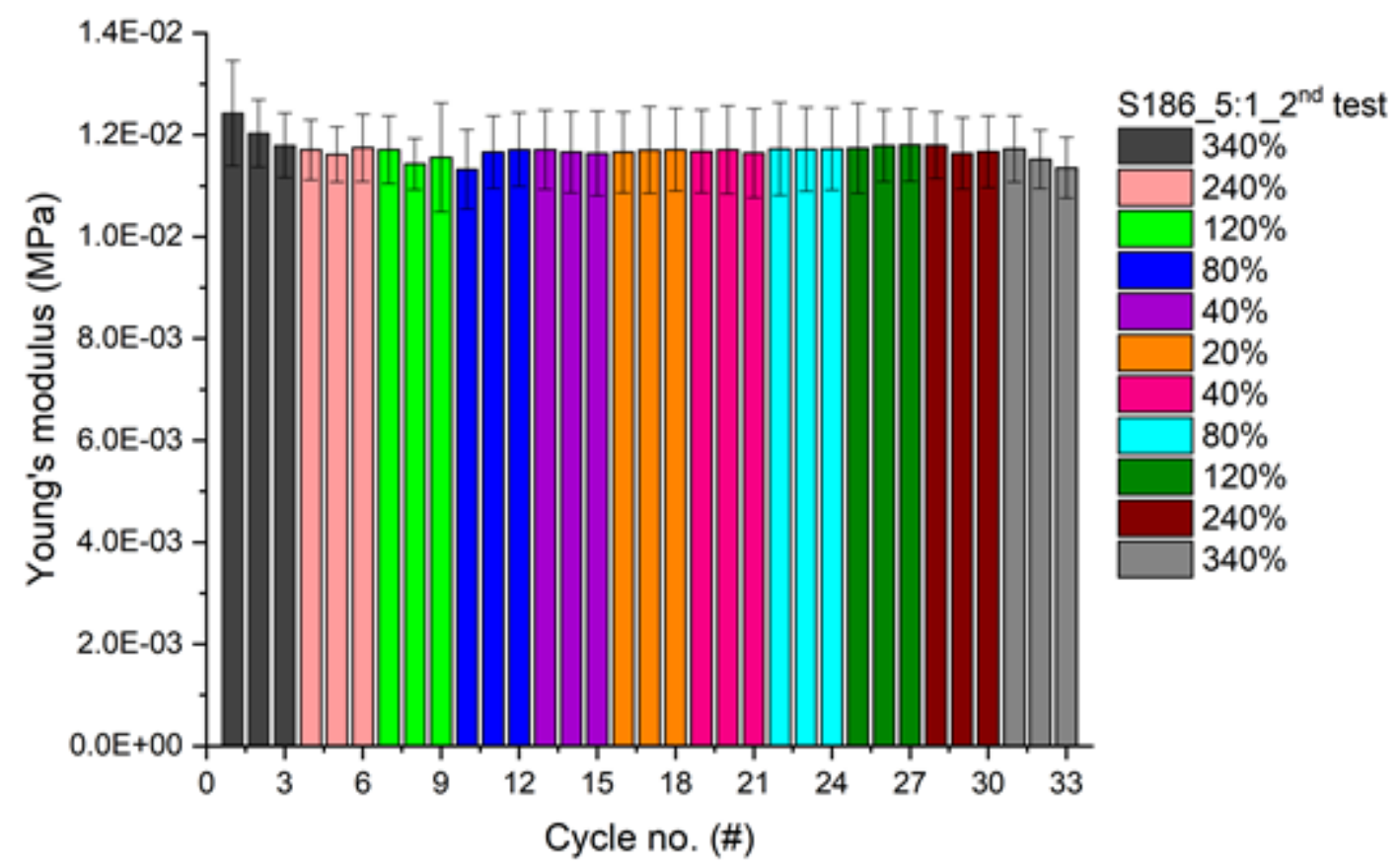

Figure 22S. Young's modulus (at 10\% strain) as a function of cycle number for different strains of S186_5:1 subjected to a $2^{\text {nd }}$ test. After cycles 16-18, the strain path was reversed. 


\section{WILEY-VCH}

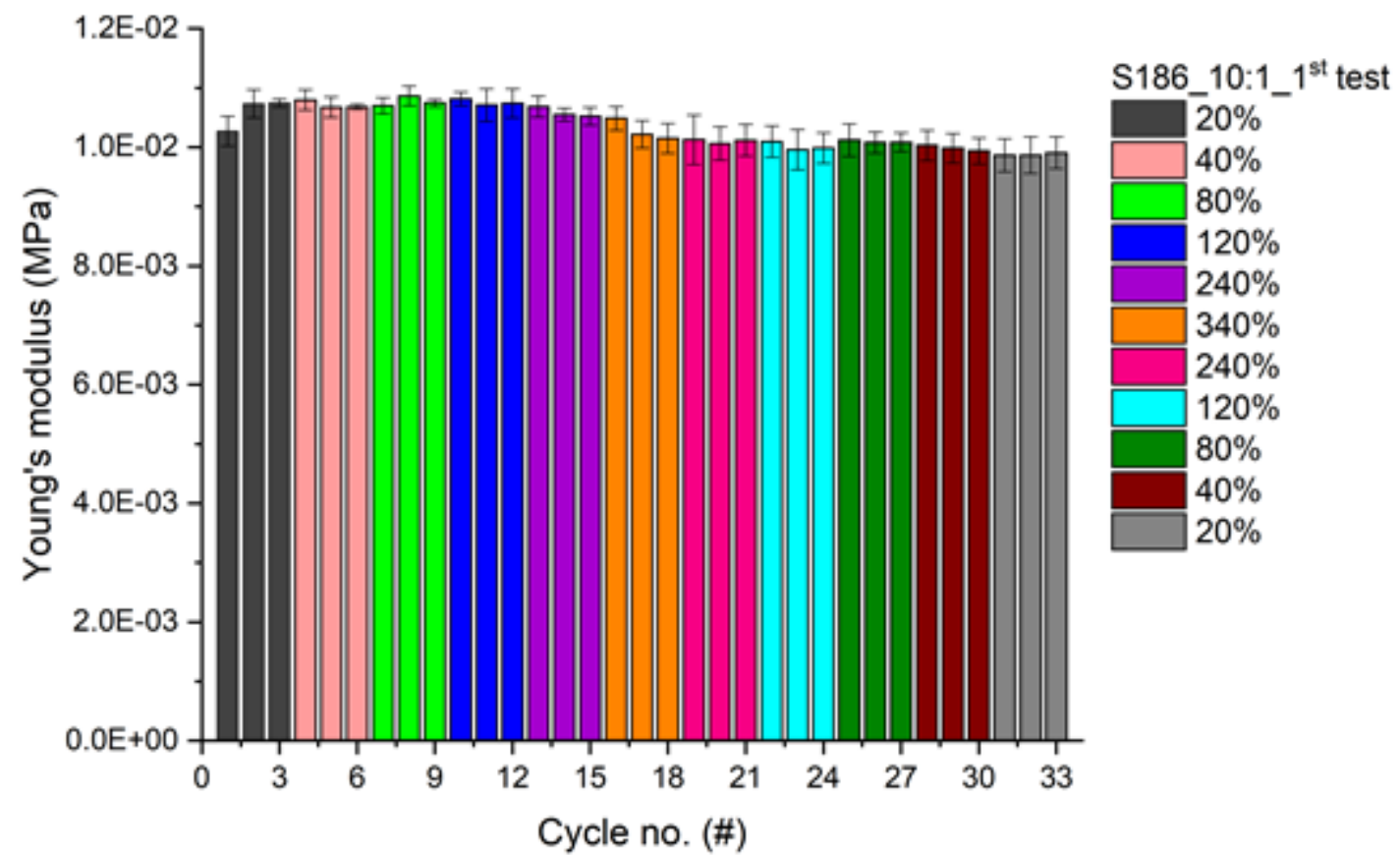

Figure 23S. Young's modulus (at 10\% strain) as a function of cycle number for different strains of S186_10:1 subjected to a $1^{\text {st }}$ test. After cycles 16-18, the strain path was reversed.

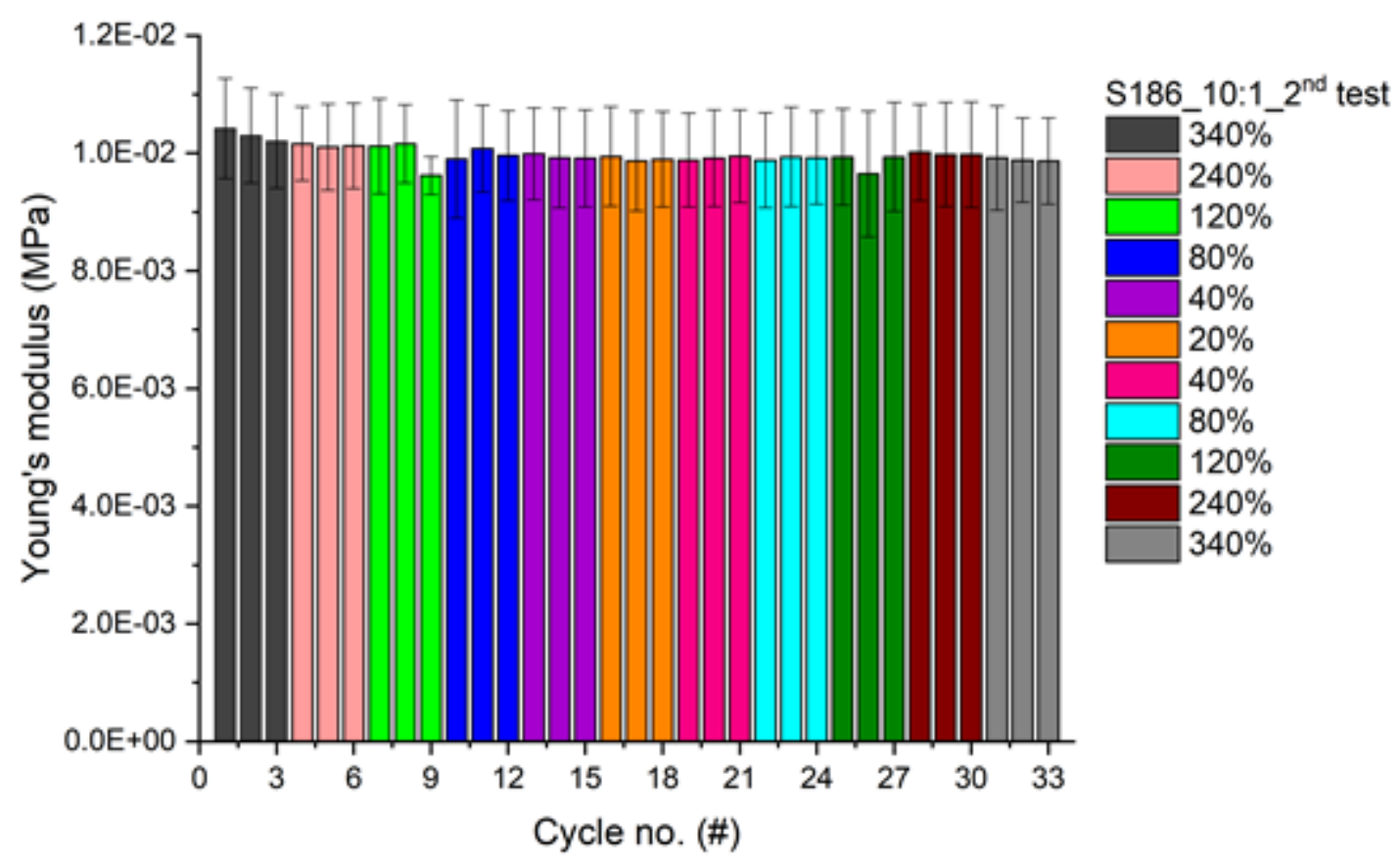

Figure 24S. Young's modulus (at 10\% strain) as a function of cycle number for different strains of S186_10:1 subjected to a $2^{\text {nd }}$ test. After cycles $16-18$, the strain path was reversed. 


\section{WILEY-VCH}

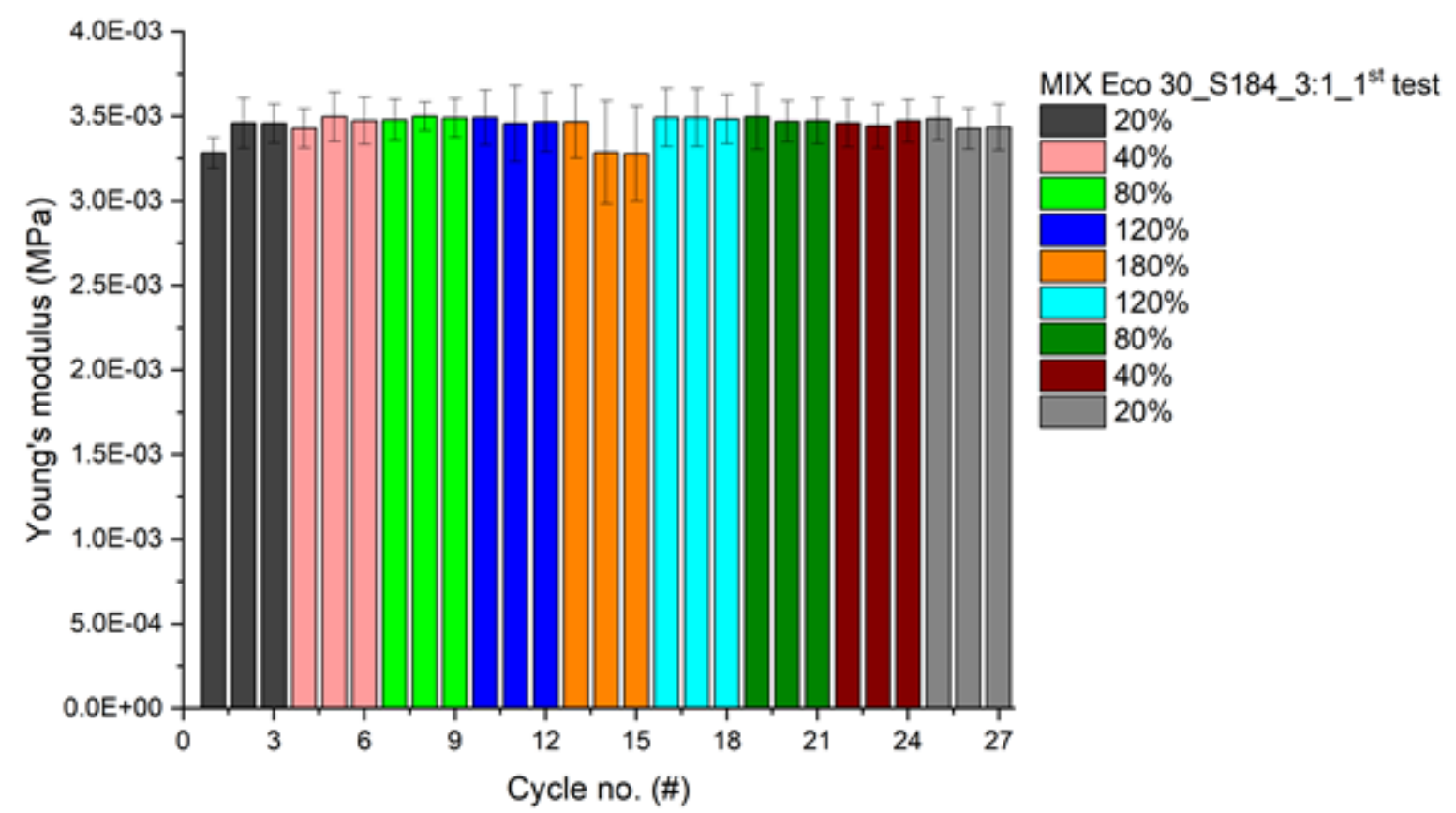

Figure 25S. Young's modulus (at 10\% strain) as a function of cycle number for different strains of MIX Eco 30S184_3:1 subjected to a $1^{\text {st }}$ test. After cycles 13-15, the strain path was reversed.

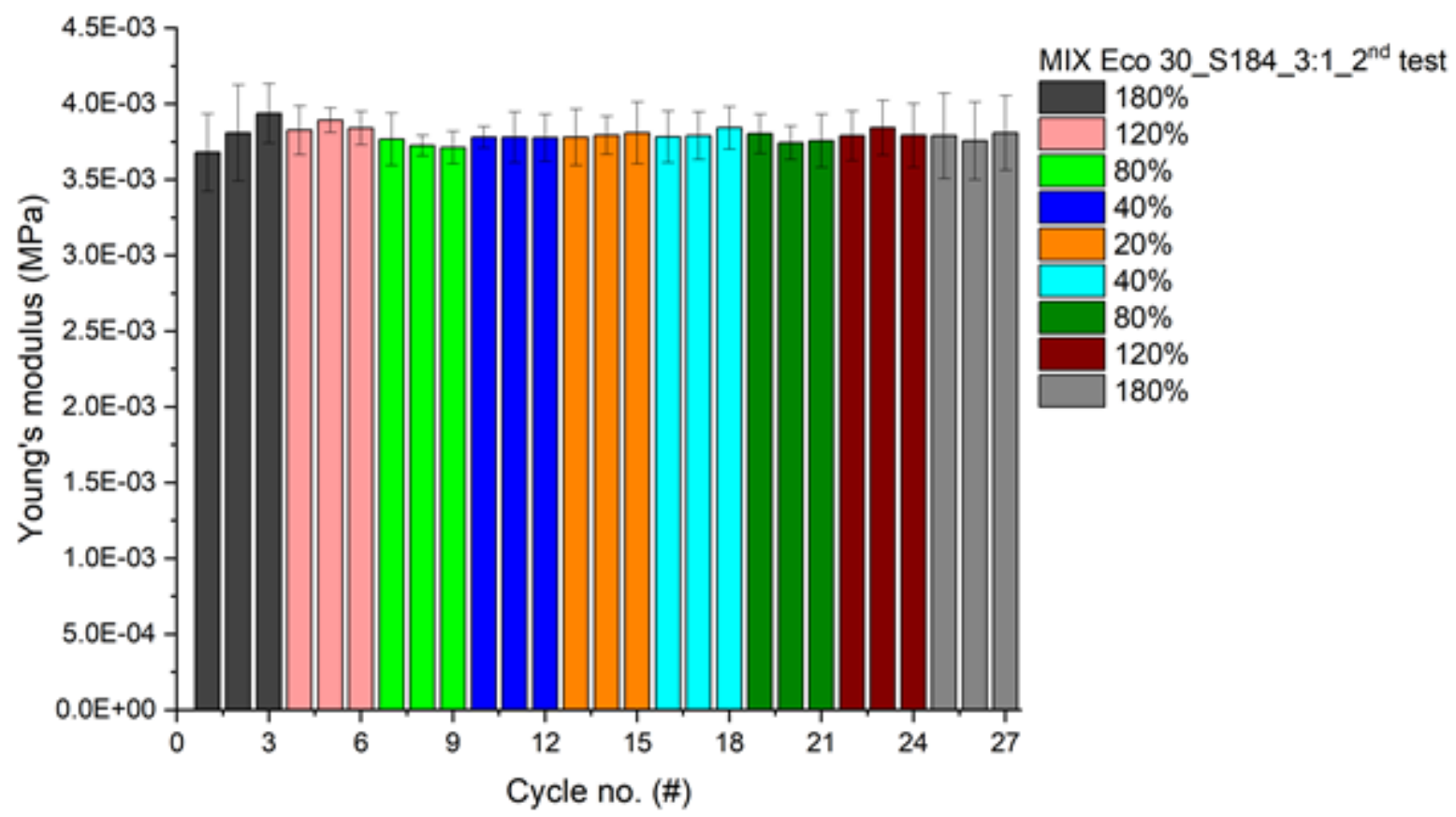

Figure 26S. Young's modulus (at 10\% strain) as a function of cycle number for different strains of MIX Eco 30_S184_3:1 subjected to a $2^{\text {nd }}$ test. After cycles 13-15, the strain path was reversed. 


\section{WILEY-VCH}

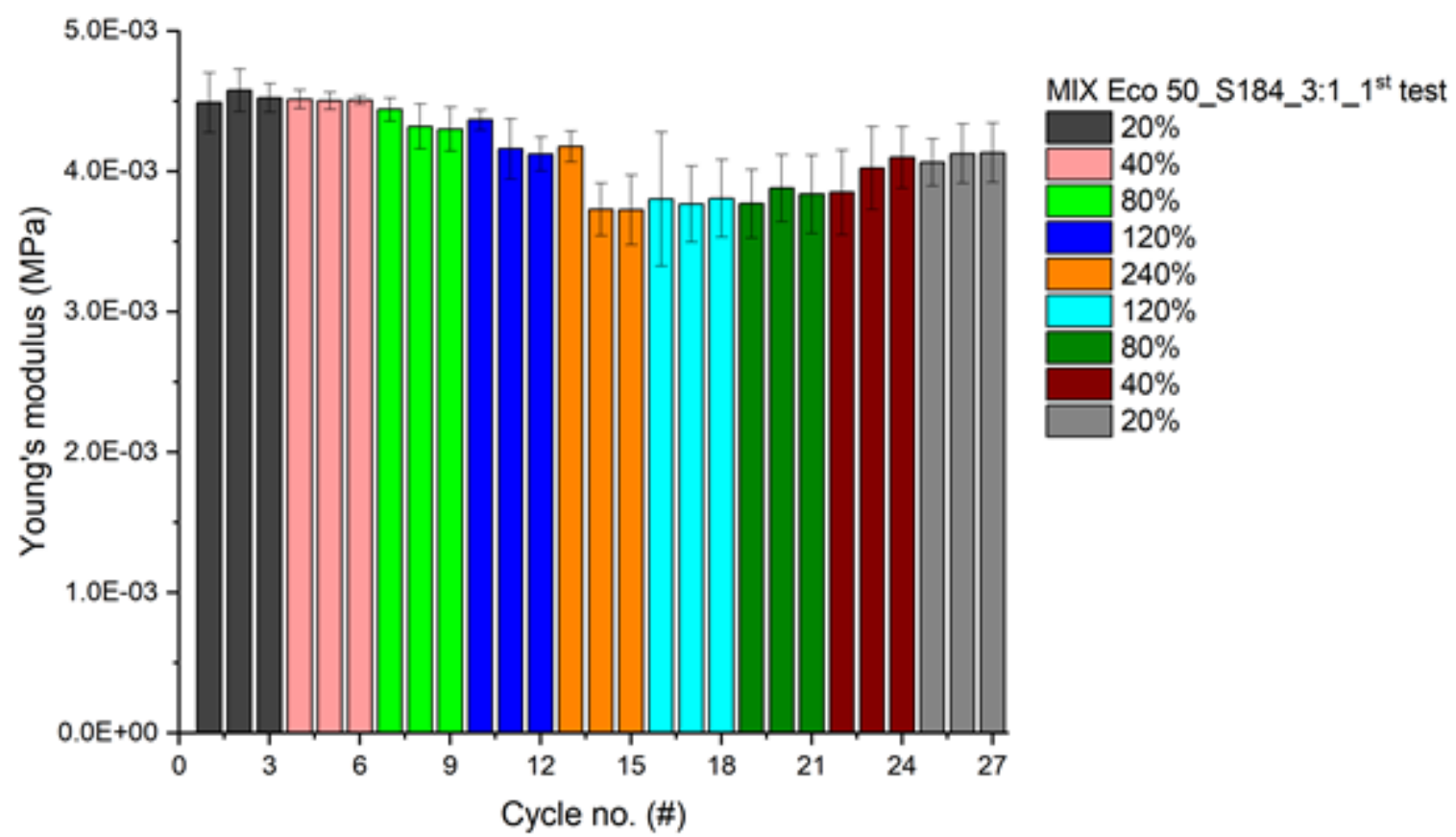

Figure 27S. Young's modulus (at 10\% strain) as a function of cycle number for different strains of MIX Eco 50_S184_3:1 subjected to a $1^{\text {st }}$ test. After cycles 13-15, the strain path was reversed.

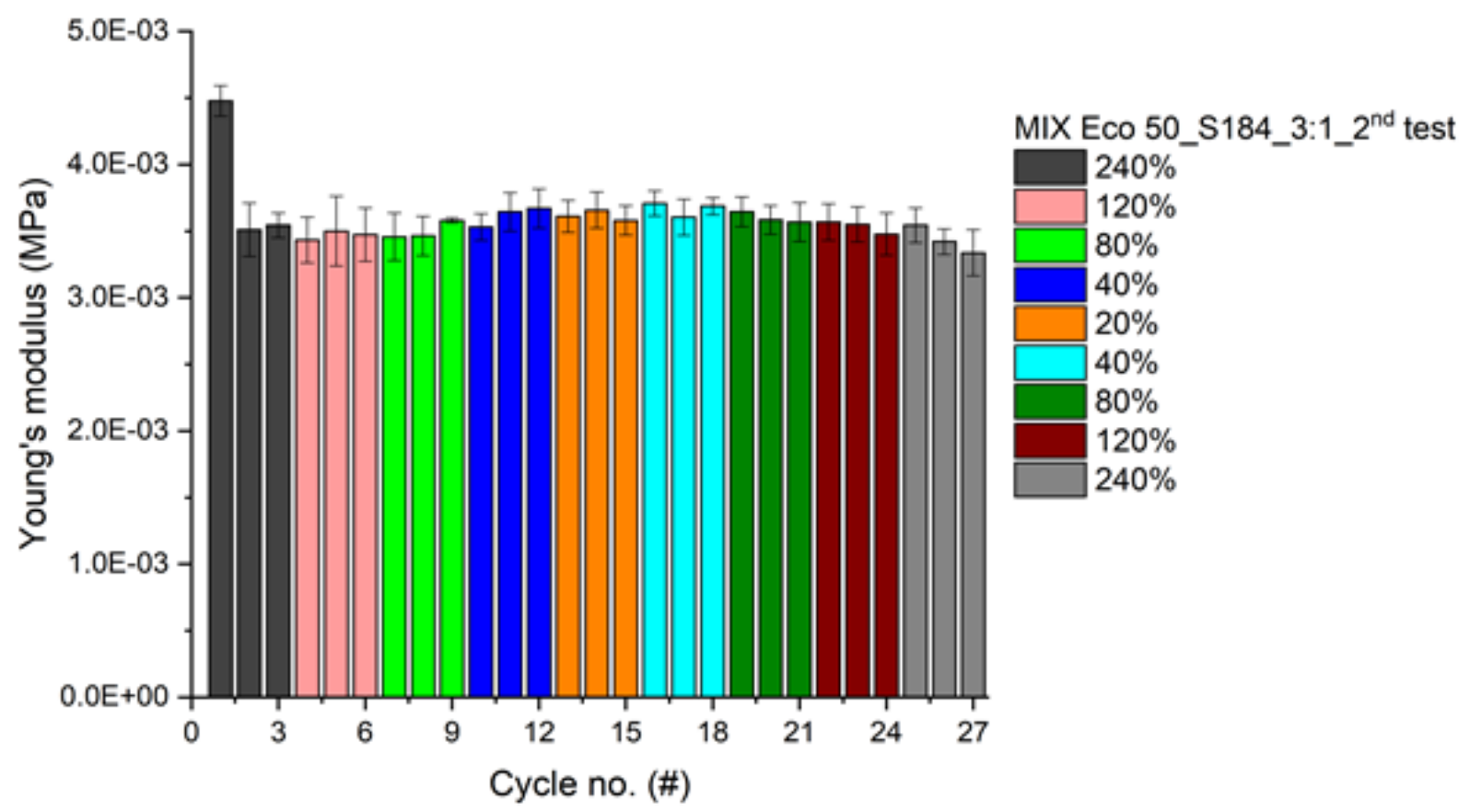

Figure 28S. Young's modulus (at 10\% strain) as a function of cycle number for different strains of MIX Eco 50_S184_3:1 subjected to a $2^{\text {nd }}$ test. After cycles 13-15, the strain path was reversed. 


\section{WILEY-VCH}

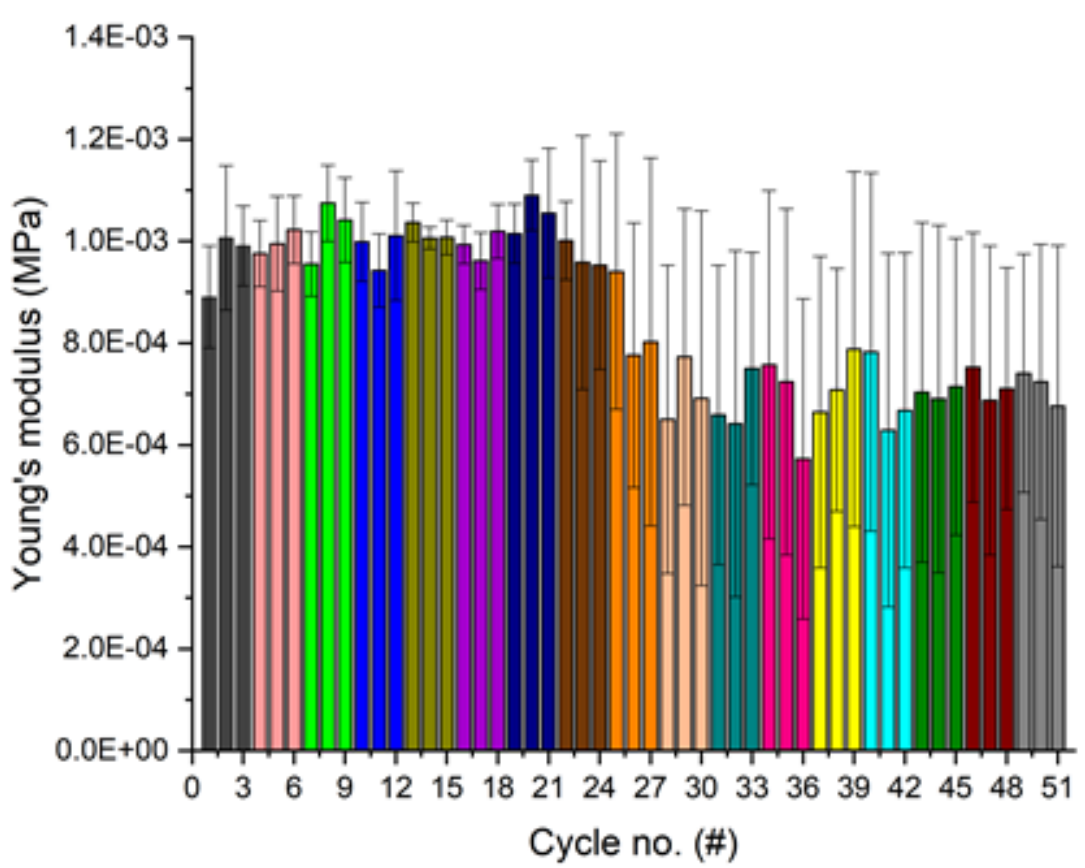

Eco 30_1 ${ }^{\text {st }}$ test

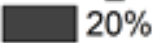
$40 \%$ $80 \%$ $120 \%$ $240 \%$ $360 \%$ $480 \%$ $600 \%$ $680 \%$ $600 \%$ $480 \%$ $360 \%$ $240 \%$ $120 \%$ $80 \%$ $40 \%$ $20 \%$

Figure 29S. Young's modulus (at 10\% strain) as a function of cycle number for different strains of Eco 30 subjected to a $1^{\text {st }}$ test. After cycles 25-27, the strain path was reversed.

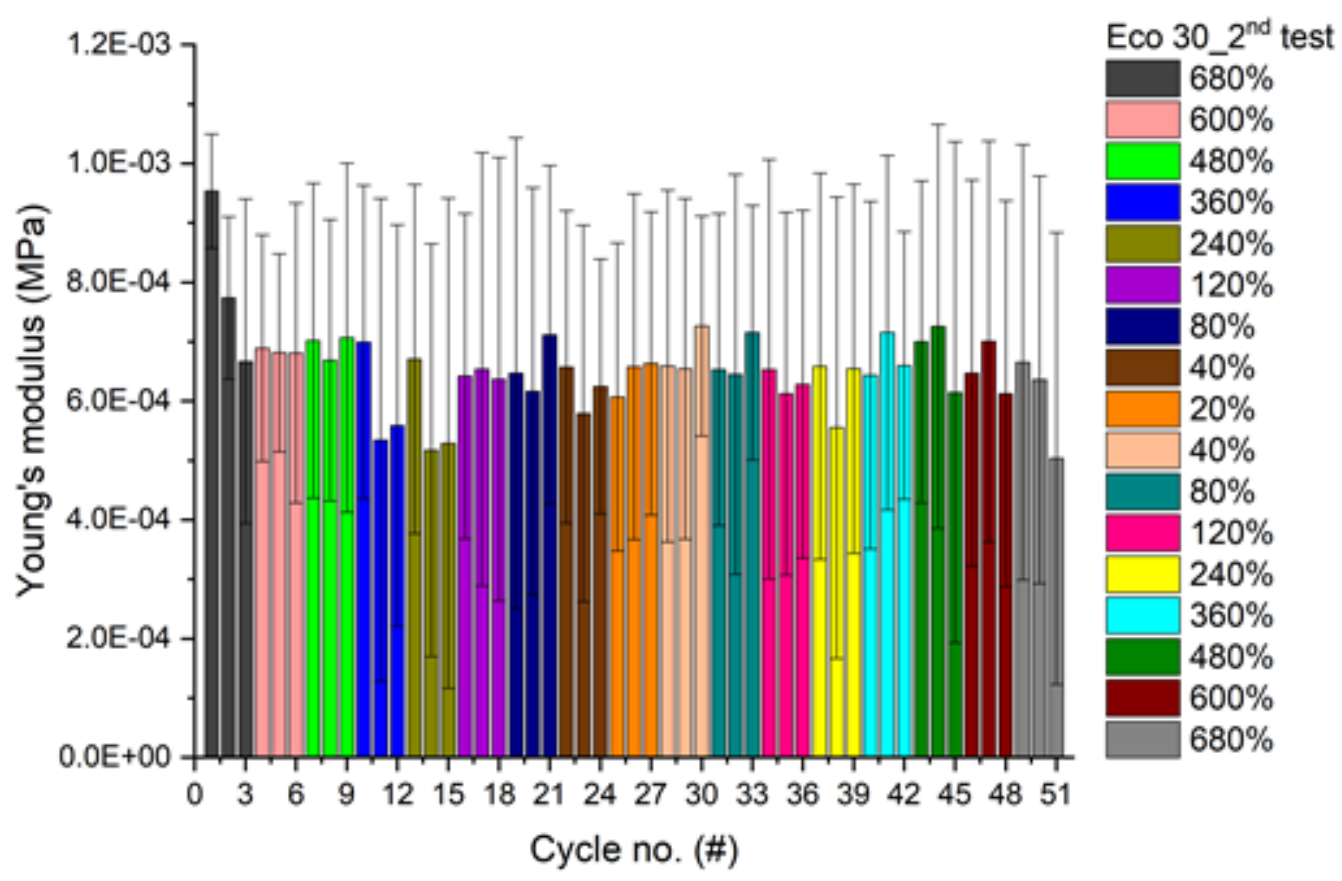

Figure 30S. Young's modulus (at 10\% strain) as a function of cycle number for different strains of Eco 30 subjected to a $2^{\text {nd }}$ test. After cycles 25-27, the strain path was reversed. 


\section{WILEY-VCH}

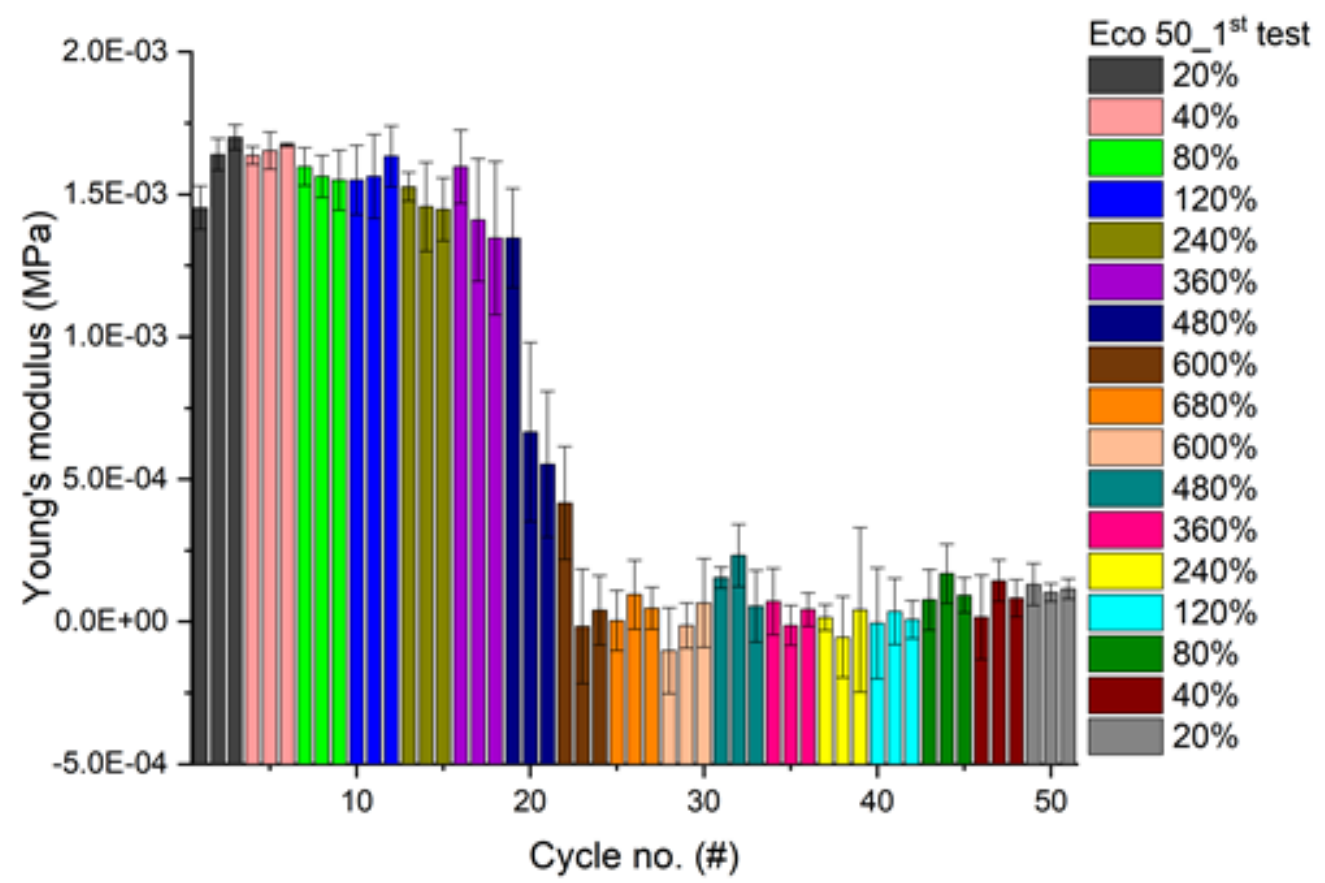

Figure 31S. Young's modulus (at 10\% strain) as a function of cycle number for different strains of Eco 50 subjected to a $1^{\text {st }}$ test. After cycles 25-27, the strain path was reversed.

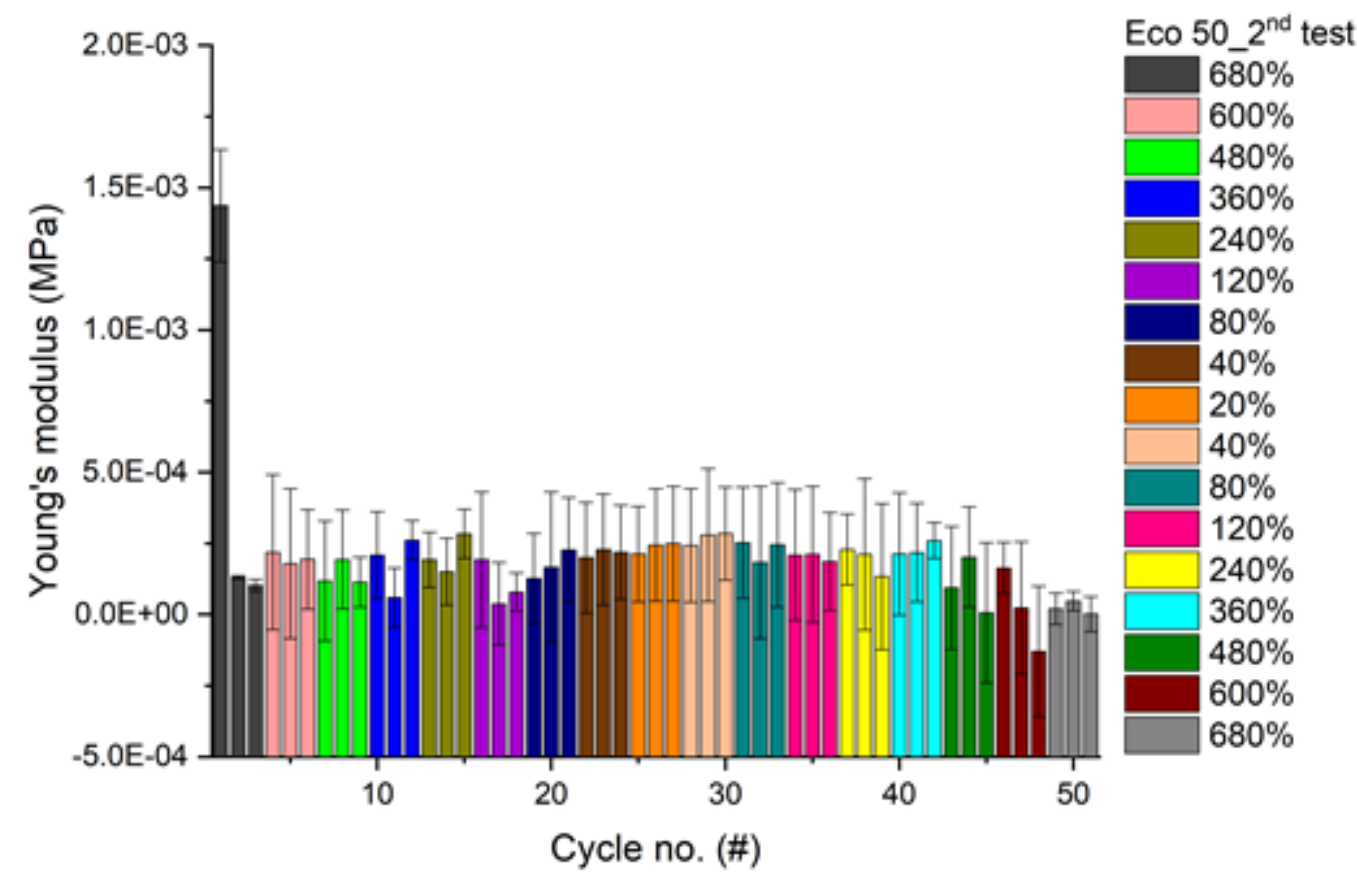

Figure 32S. Young's modulus (at 10\% strain) as a function of cycle number for different strains of Eco 50 subjected to a $2^{\text {nd }}$ test. After cycles 25-27, the strain path was reversed. 
WILEY-VCH

a)

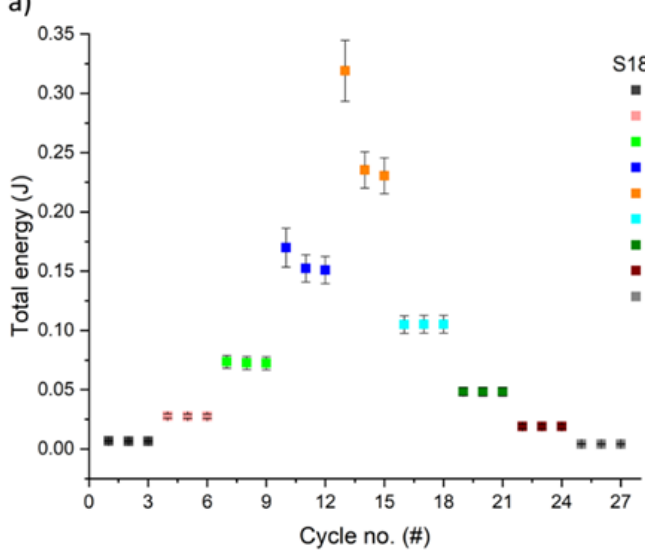

b)

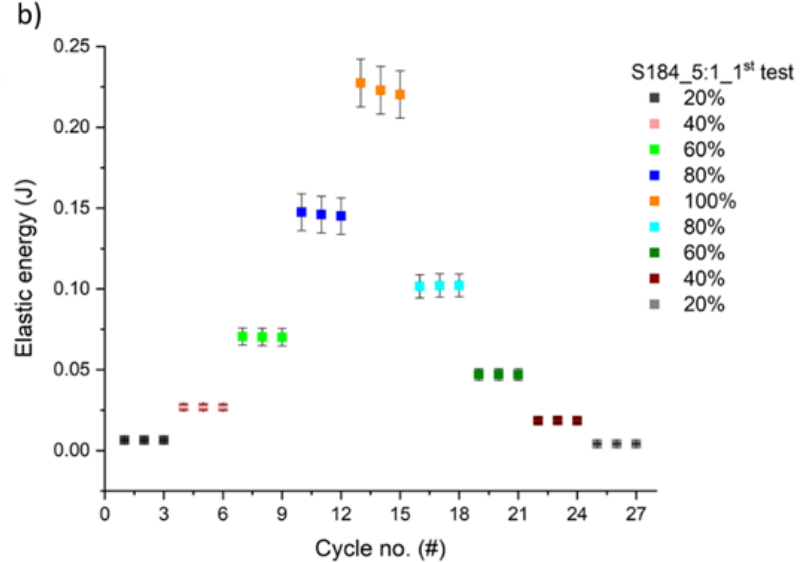

Figure 33S. Total energy a) and elastic energy b) as a function of cycle number for different strains of S184_5:1 submitted to a $1^{\text {st }}$ test. After cycles 13-15, the strain was reversed.

a)

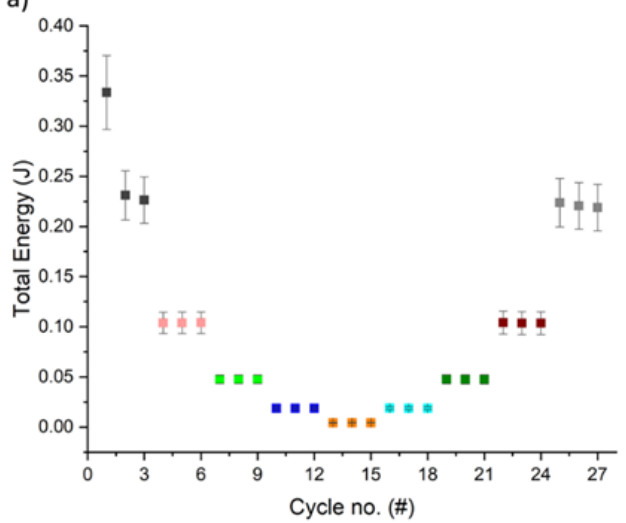

b)

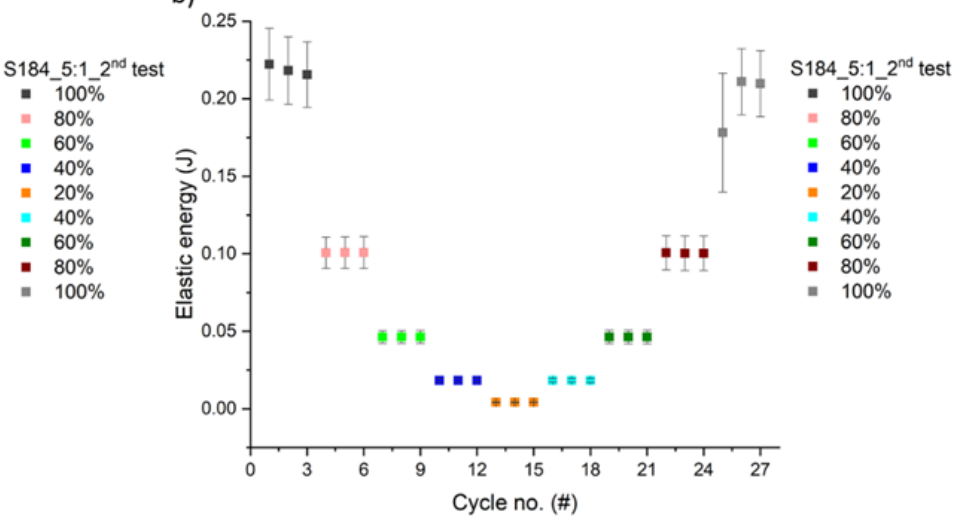

Figure 34S. Total energy a) and elastic energy b) as a function of cycle number for different strains of S184_5:1 submitted to a $2^{\text {nd }}$ test. After cycles 13-15, the strain was reversed.

a)

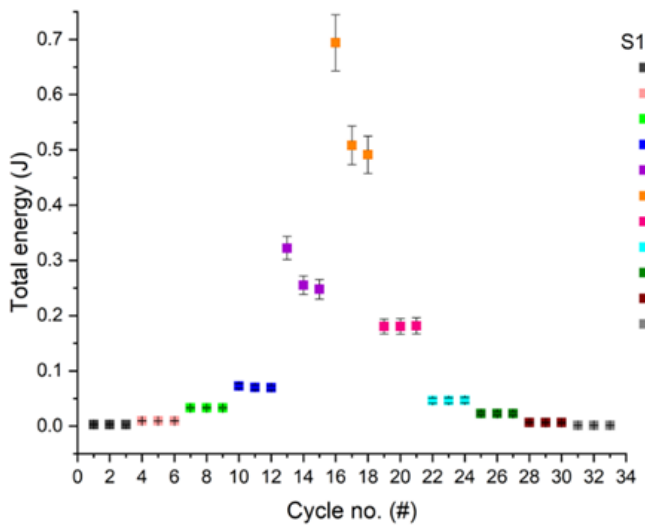

b)

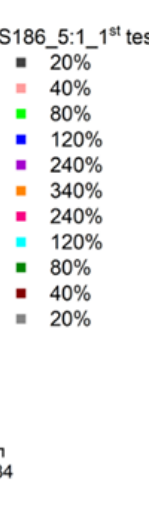

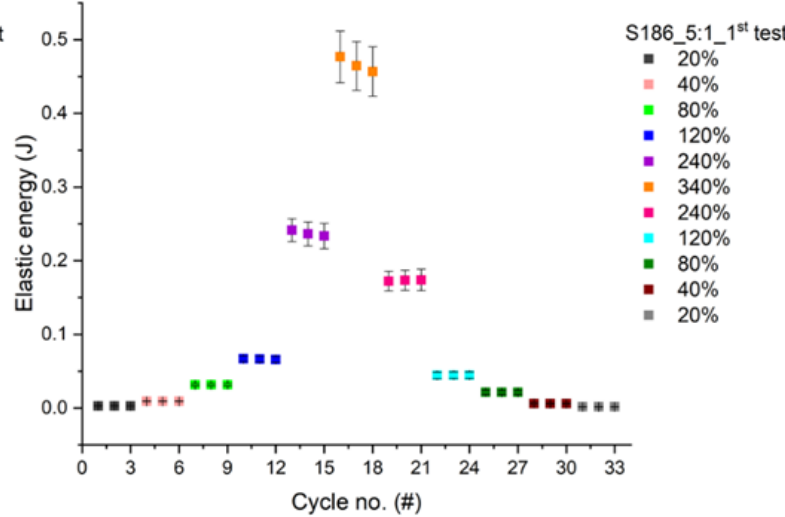

Figure 35S. Total energy a) and elastic energy b) as a function of cycle number for different strains of S186_5:1 submitted to a $1^{\text {st }}$ test. After cycles 16-18, the strain was reversed. 
WILEY-VCH

a)

b)
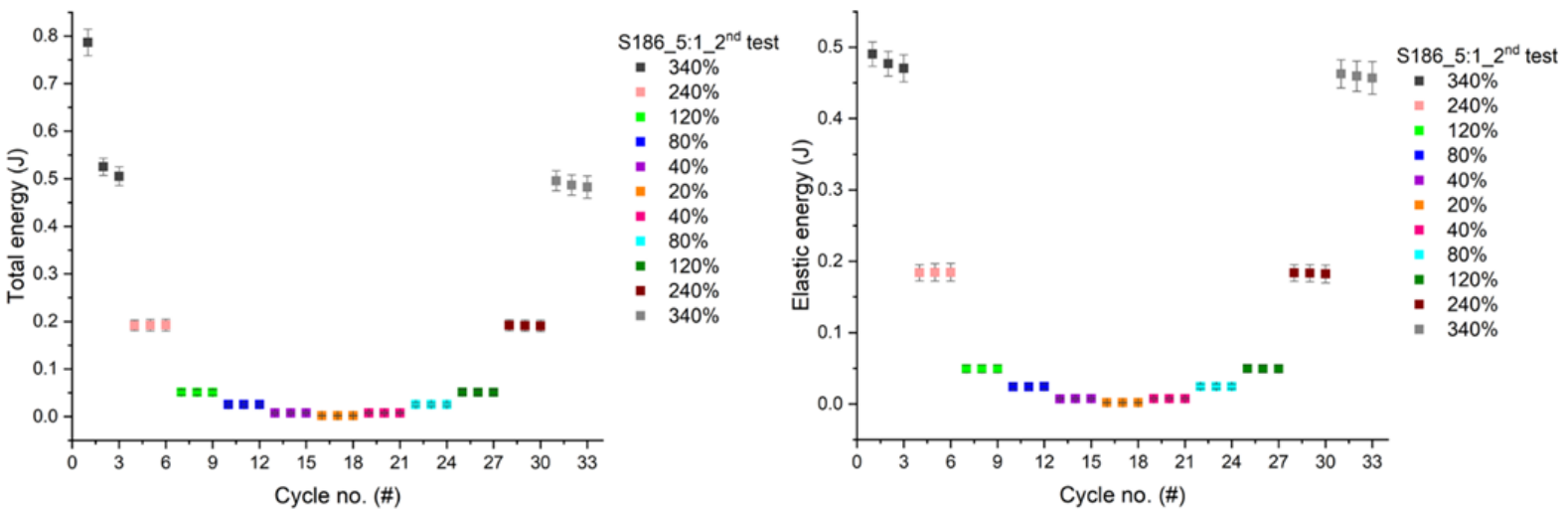

Figure 36S. Total energy a) and elastic energy b) as a function of cycle number for different strains of S186_5:1 submitted to a $2^{\text {nd }}$ test. After cycles 16-18, the strain was reversed.

b)

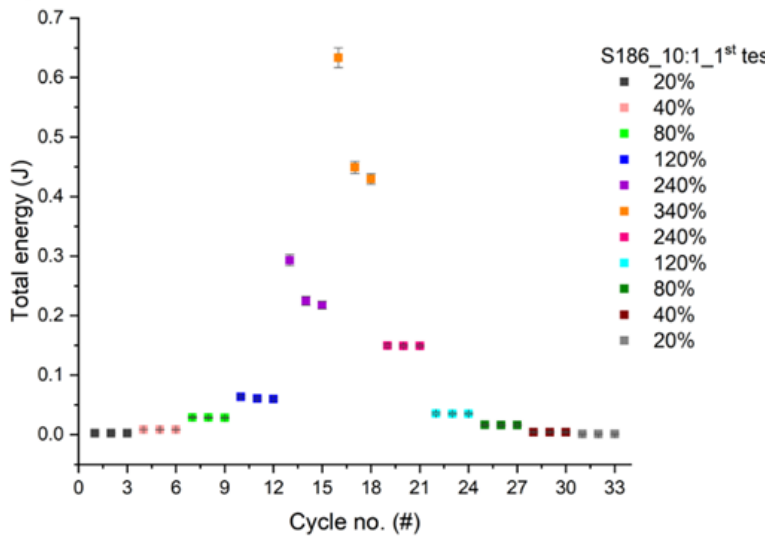

a)

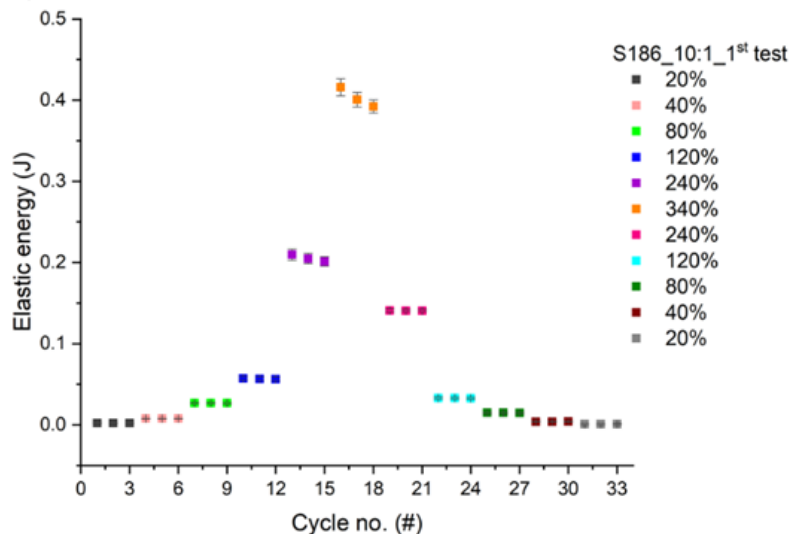

Figure 37S. Total energy a) and elastic energy b) as a function of cycle number for different strains of S186_10:1 submitted to a $1^{\text {st }}$ test. After cycles $16-18$, the strain was reversed.

a)

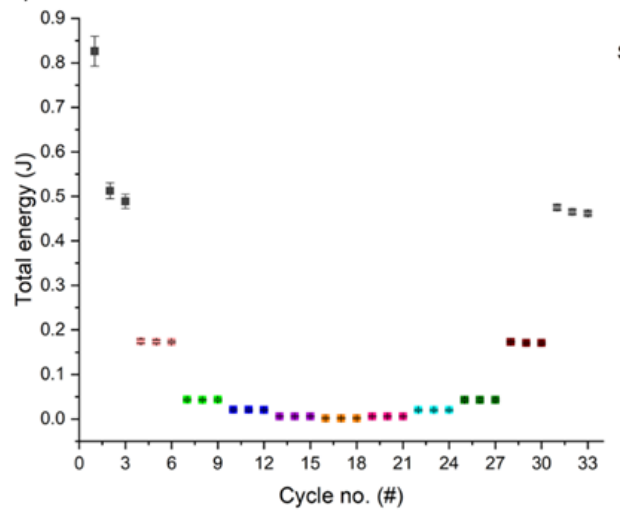

b)

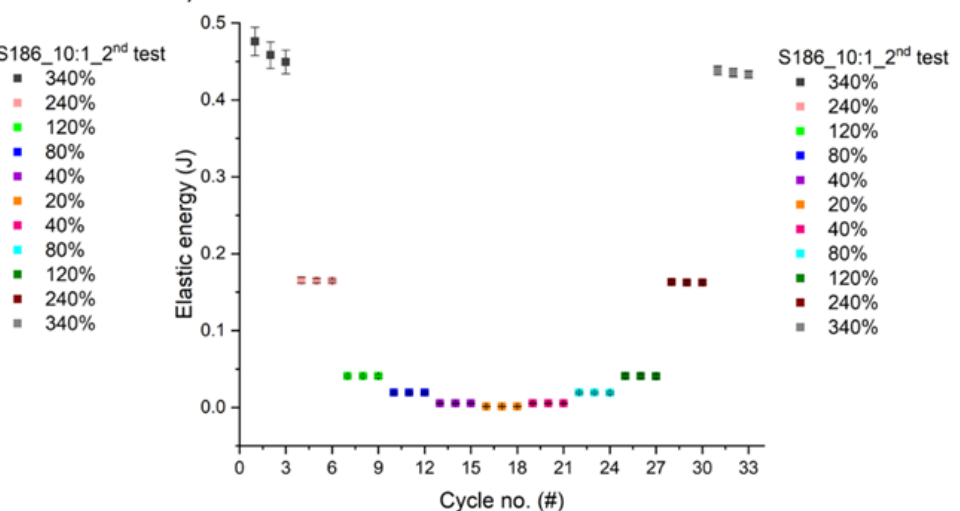

Figure 38S. Total energy a) and elastic energy b) as a function of cycle number for different strains of S186_10:1 submitted to a $2^{\text {nd }}$ test. After cycles 16-18, the strain was reversed. 
WILEY-VCH

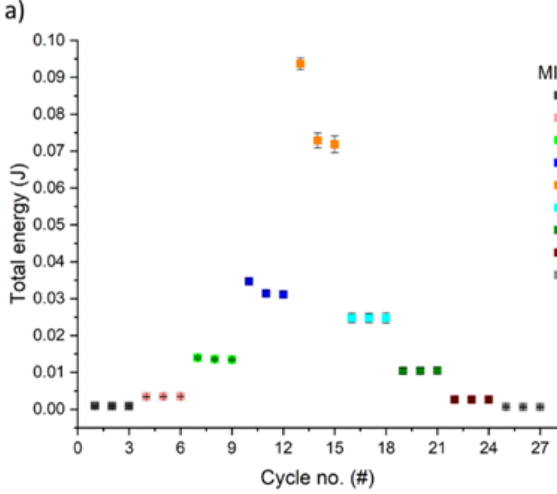

b)

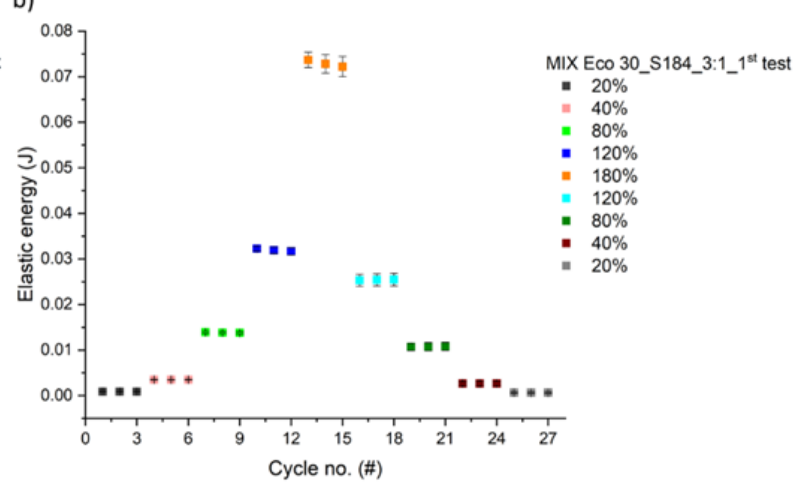

Figure 39S. Total energy a) and elastic energy b) as a function of cycle number for different strains of MIX Eco 30_S184_3:1 submitted to a $1^{\text {st }}$ test. After cycles 13-15, the strain was reversed.
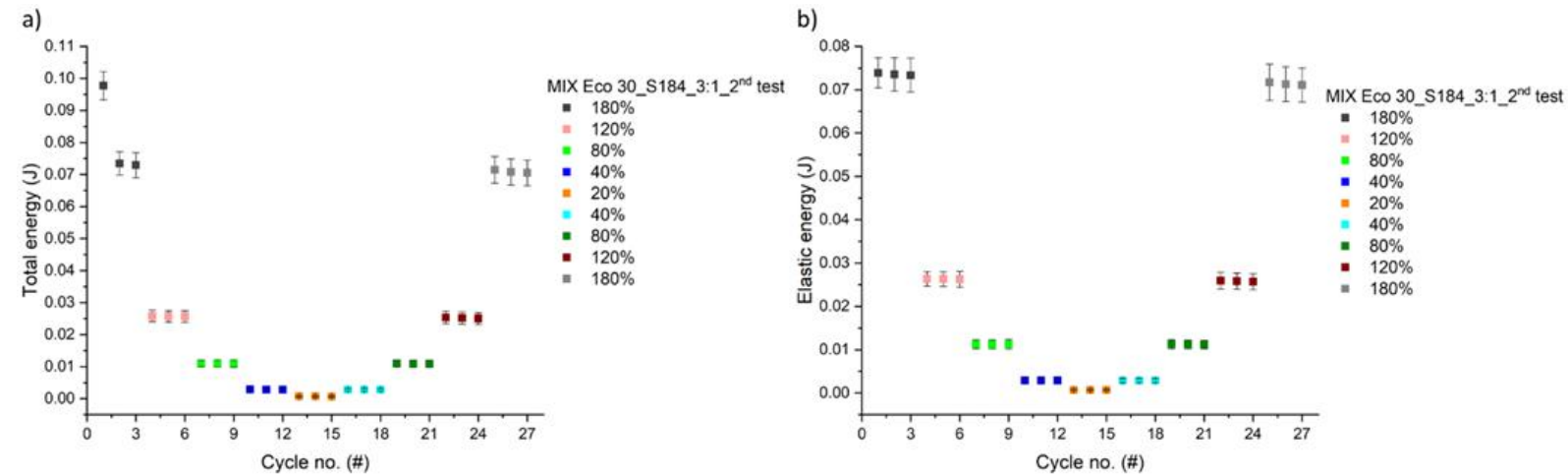

Figure 40S. Total energy a) and elastic energy b) as a function of cycle number for different strains of MIX Eco 30_S184_3:1 submitted to a $2^{\text {nd }}$ test. After cycles 13-15, the strain was reversed.

a)

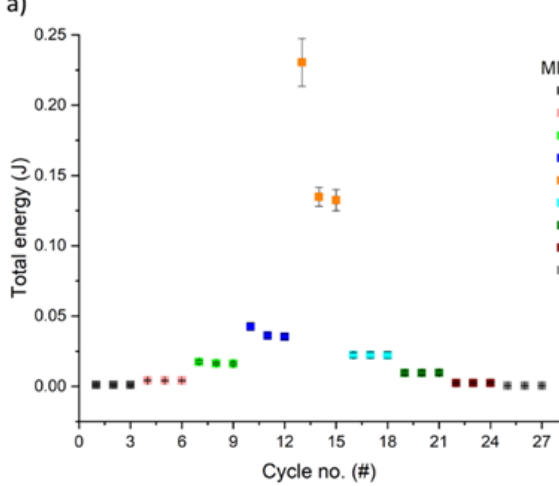

b)

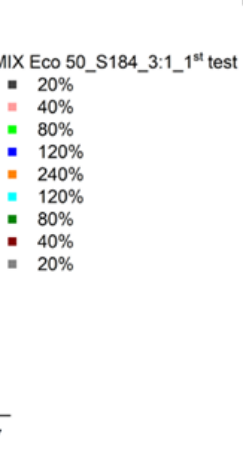

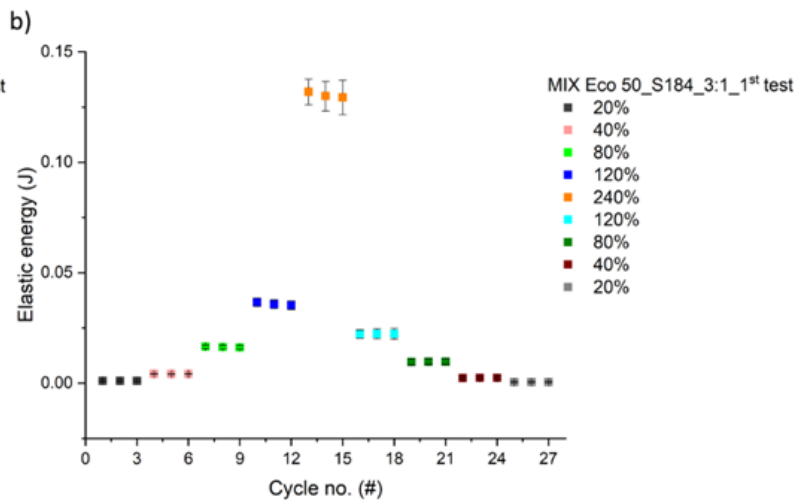

Figure 41S. Total energy a) and elastic energy b) as a function of cycle number for different strains of MIX Eco 50_S184_3:1 submitted to a $1^{\text {st }}$ test. After cycles 13-15, the strain was reversed. 
WILEY-VCH

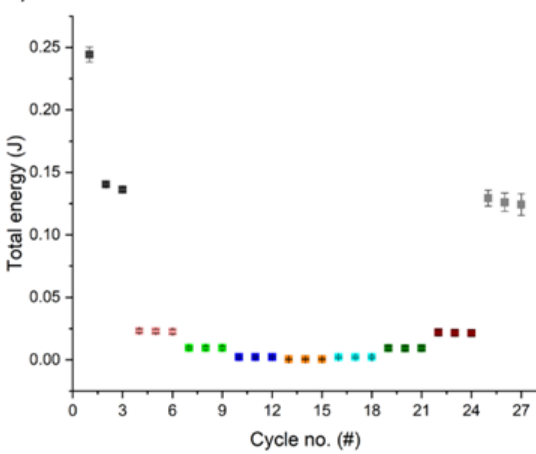

b)

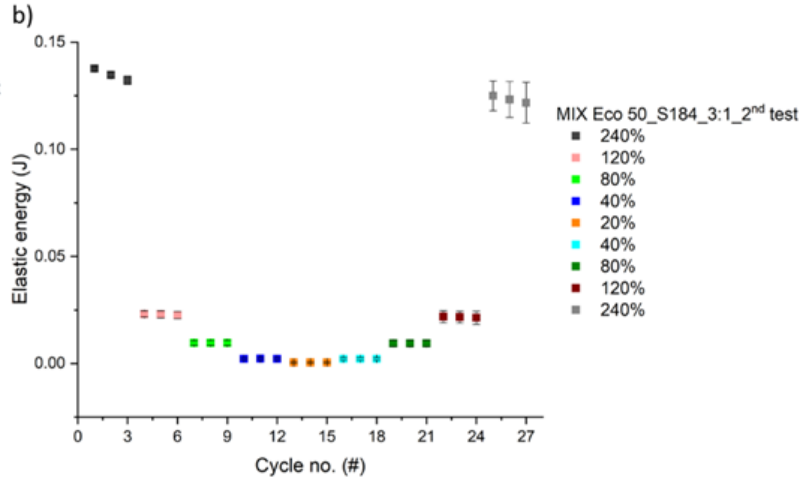

Figure 42S. Total energy a) and elastic energy b) as a function of cycle number for different strains of MIX Eco 50_S184_3:1 submitted to a $2^{\text {nd }}$ test. After cycles 13-15, the strain was reversed.

a)

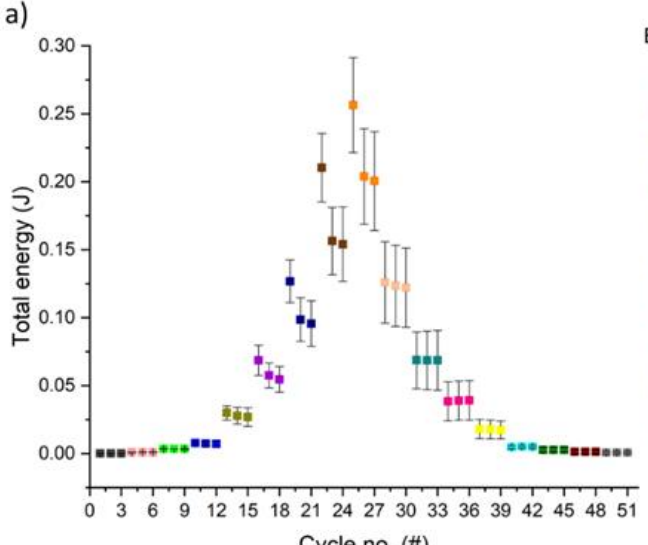

b)
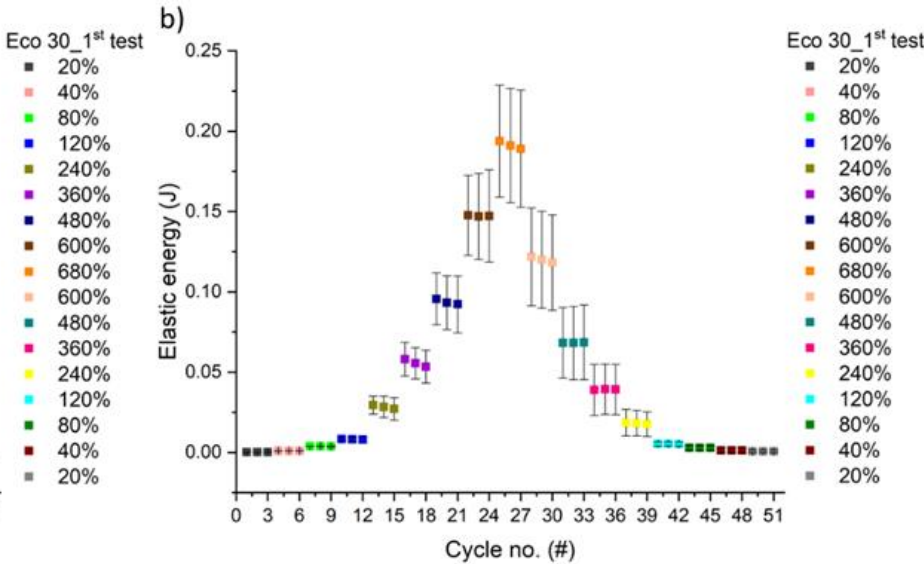

Figure 43S. Total energy a) and elastic energy b) as a function of cycle number for different strains of Eco 30 submitted to a $1^{\text {st }}$ test. After cycles 25-27, the strain was reversed.

a)

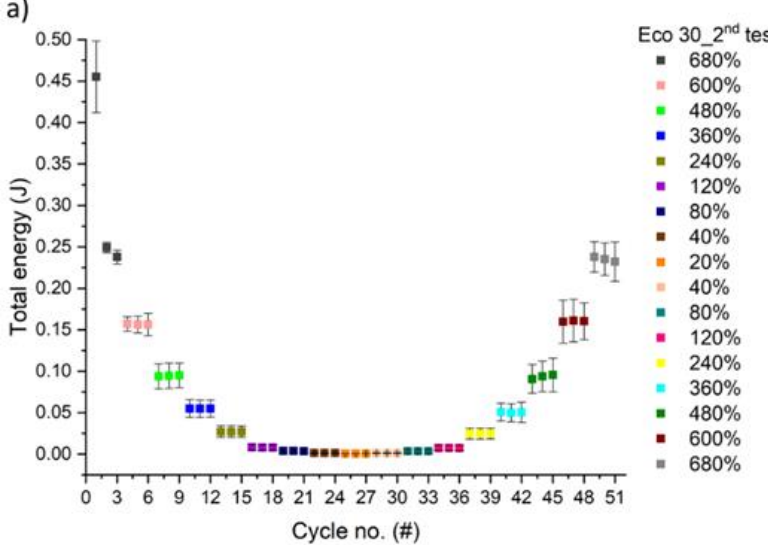

b)

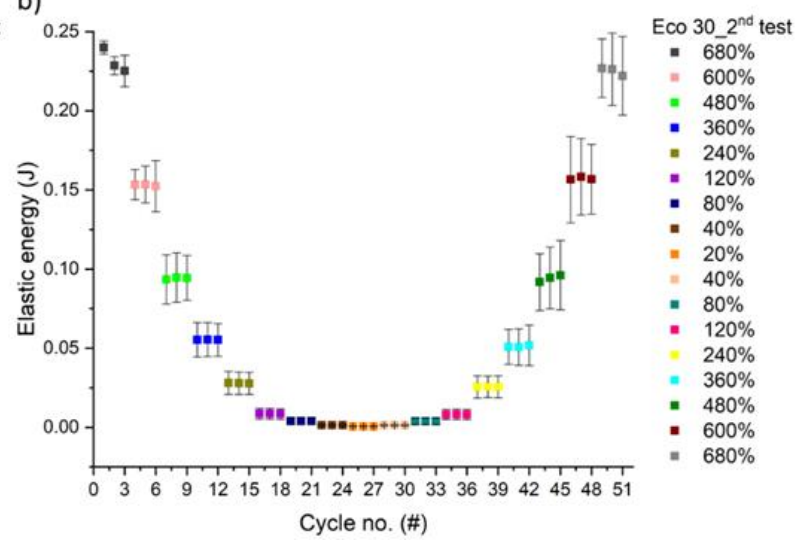

Figure 44S. Total energy a) and elastic energy b) as a function of cycle number for different strains of Eco 30 submitted to a $2^{\text {nd }}$ test. After cycles 25-27, the strain was reversed. 


\section{WILEY-VCH}
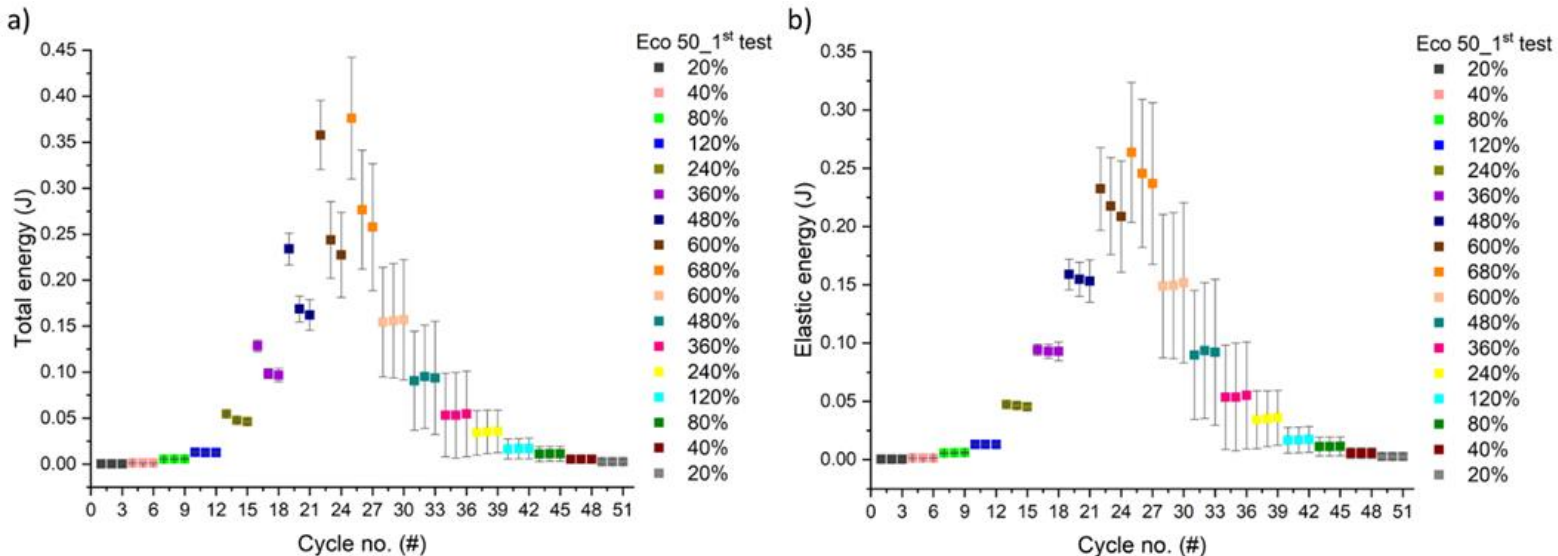

Figure 45S. Total energy a) and elastic energy b) as a function of cycle number for different strains of Eco 50 submitted to a $1^{\text {st }}$ test. After cycles $25-27$, the strain was reversed.

a)

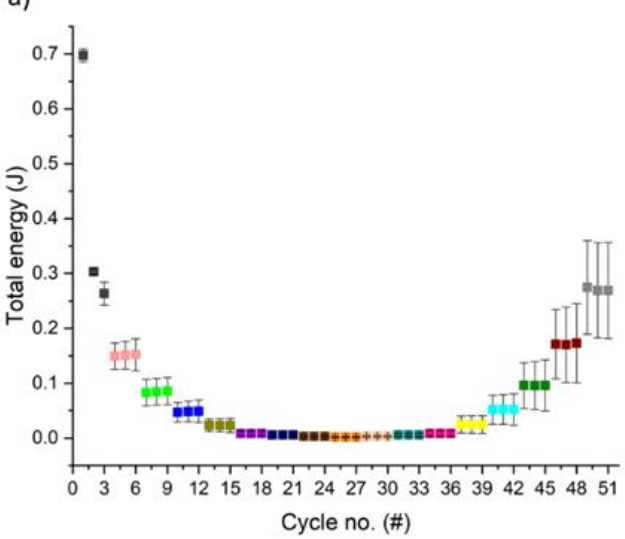

b)

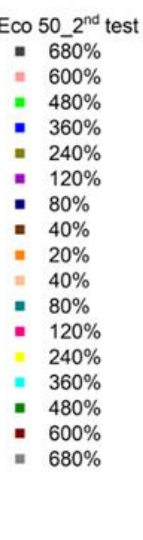

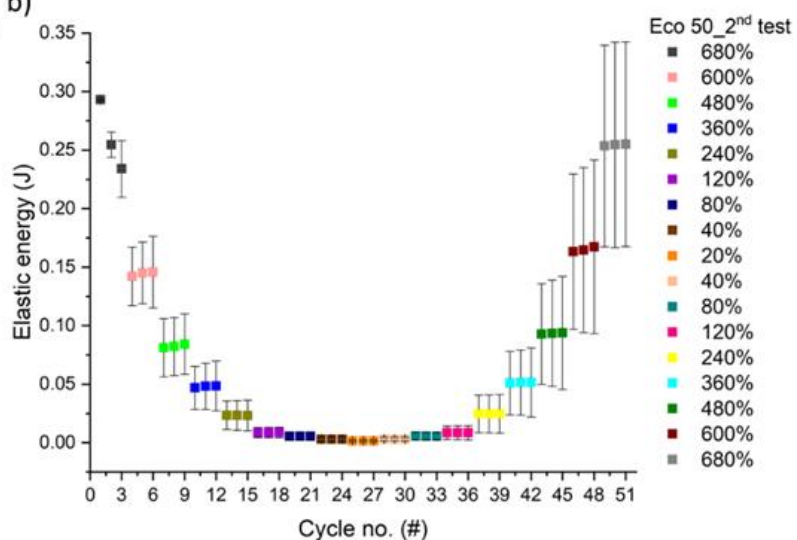

Figure 46S. Total energy a) and elastic energy b) as a function of cycle number for different strains of Eco 50 submitted to a $2^{\text {nd }}$ test. After cycles 25-27, the strain was reversed.

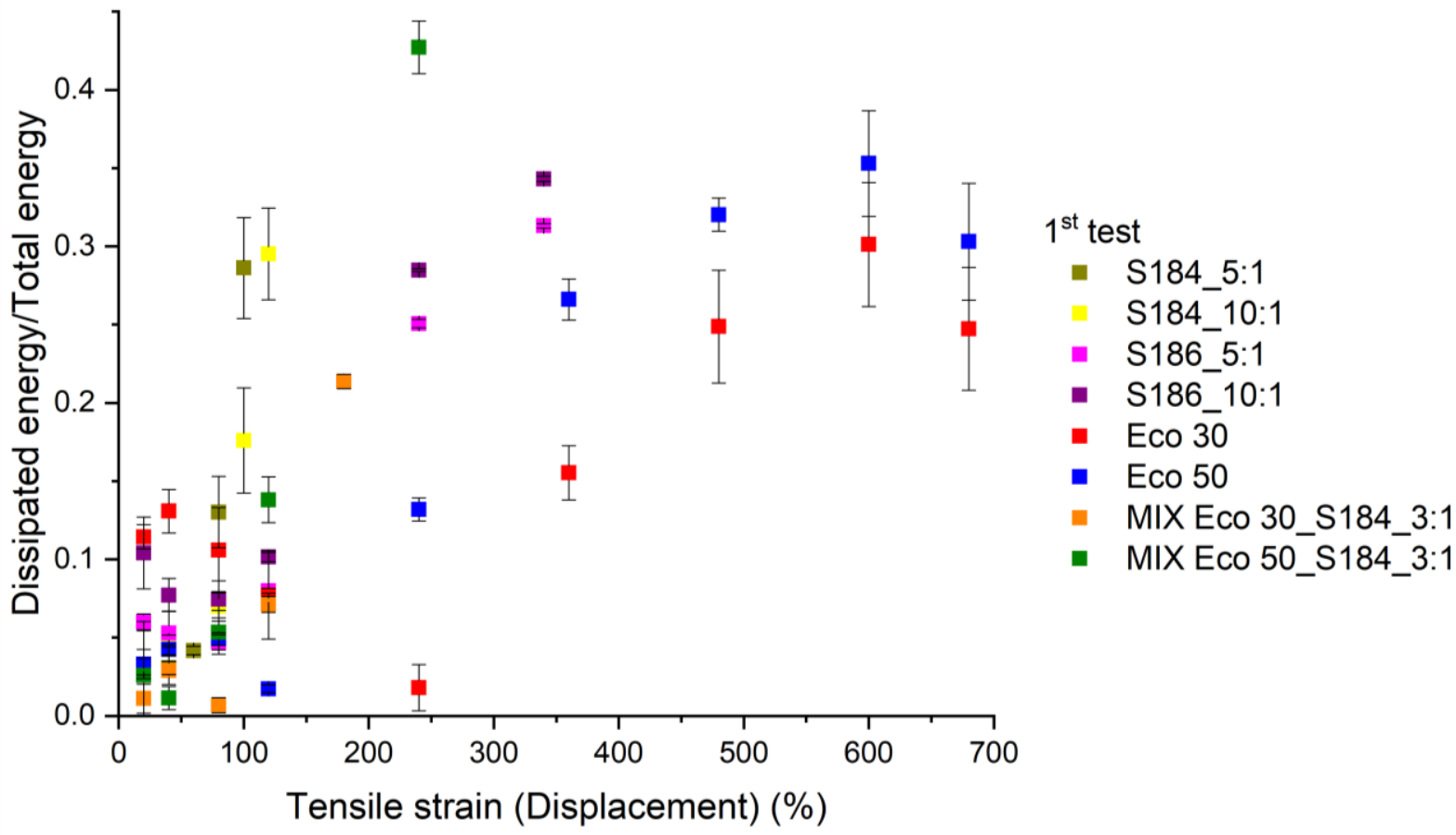

Figure 47S. Dissipated energy normalized to total energy of different silicone formulations in every first cycle at different strains applied in the $1^{\text {st }}$ test. 

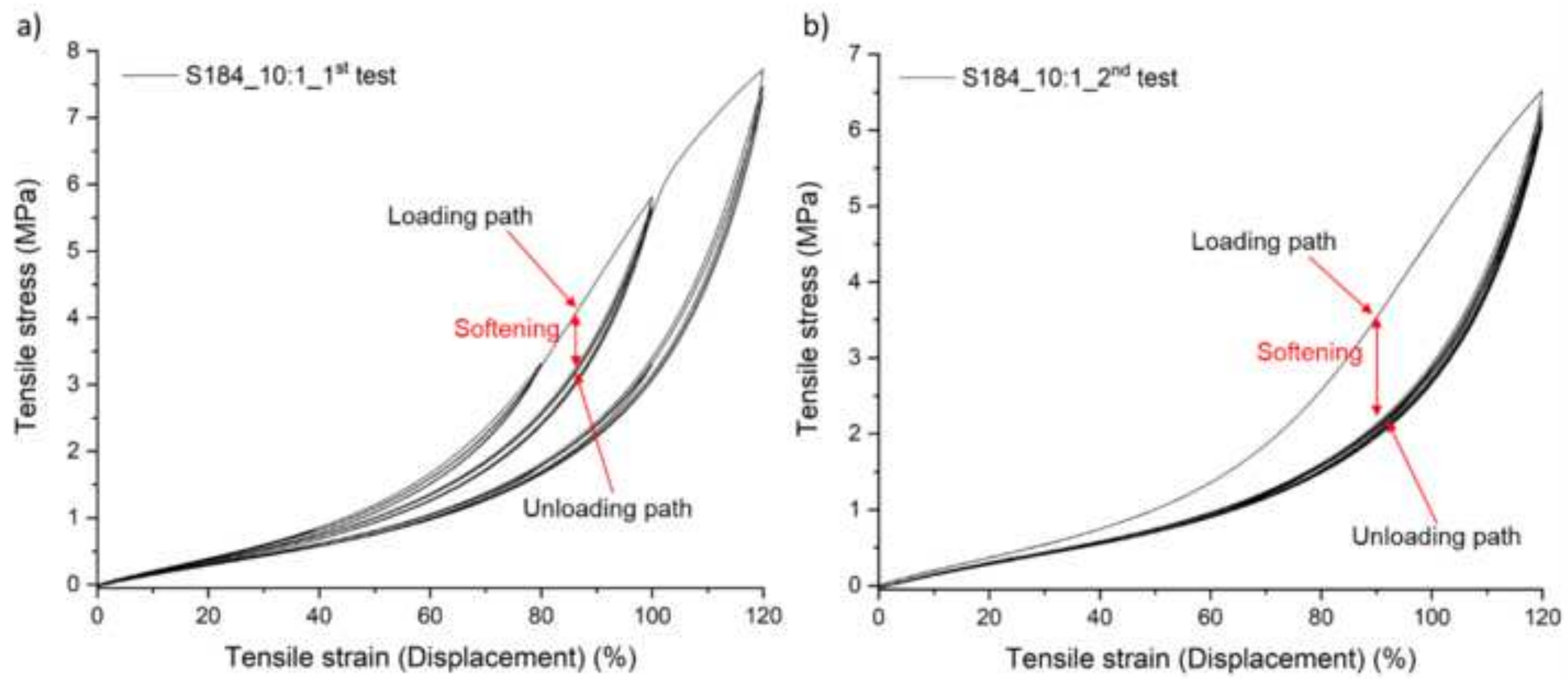
Response to the reviewers

Manuscript ID: No. adem.202000687

Dear editor, dear reviewers,

We highly appreciate the constructive feedback from the reviewers. We would like to thank to the reviewers for suggesting relevant literature for this manuscript. We have taken feedback and all suggestions into account and implemented the changes in the manuscript, which, we believe, improved the manuscript greatly.

Below we have addressed all raised issues one-by-one. Changes in the manuscript have a blue font color.

We hope that you will accept our revised manuscript.

\section{Reviewer \#1}

- Comments: The manuscript "Importance of Mullins effect..." by Krpovic et al described a large series of experiments on tensile tests applied to various silicone formulations. Even if I have no doubts on the veracity of the results, the interpretation of them are totally lacking. The Mullins effect is known for very long now and people still argue on the different causes of it. Most often, the network of filler particles is acknowledged to produce this effect, but it is known to occur also on unfilled elastomers. The problem in the current study is that there are no correlation between the type, and then formulation of the elastomer, and the tensile test results. We don't know how much filler they are composed of (if any), the type of inorganic particles introduced, their content and so on. A technical report like this one surely could help the community of mechanical engineering selecting the best material for future application, but the paper as it is written does not bring any novelty on the material side. I suggest that the authors take the time to describe what are the elastomers composed of and analyze again their results according to the formulations they have selected, so that to answer the following questions:

Response: Thank you for your comments. Hopefully, it will be clear from the specific answers below that our initial thoughts were about a tutorial since people in the area of soft robotics do not necessarily come with background knowledge on rubbers and thereby the Mullins effect is often - wrongly - ignored. We, however, agree with many of the comments below and that further knowledge will make this article more relevant.

- Question 1: What type of filler induces the worst Mullins effect?

Response: We made changes in the manuscript to address the question regarding filler content, and whether higher filler content induces higher Mullins effect. Since the type(s) and possible modification(s) of the fillers in formulations are not known (trade secrets), conclusions regarding which type of the filler induces worst Mullins effect was not discussed in the article. 
Addition in the article on page 5, section -Materials and sample preparation-: Since resins and inorganic fillers (most commonly silica fillers) are already present in silicone elastomer formulations, the elastomers are prepared as received from the supplier, without introduction of additional fillers.

Addition in the article on page 10, section - Analysis of the chemical characteristics of the elastomers -: Thermogravimetric analysis of cured silicone formulations was performed from room temperature to $900^{\circ} \mathrm{C}$ under nitrogen atmosphere to inspect general thermal decomposition behavior of the cured silicone elastomer formulations and obtain information on the solid residue after thermal treatment. The onset of thermal degradation is commonly predicted using the temperature at which 5\% weight loss is recorded. ${ }^{[45-47]}$ Onset temperatures of degradation, residual weights at $900^{\circ} \mathrm{C}$, and amounts of filler in each formulation are presented in Table 2. As indicated above, resins and inorganic fillers (most commonly silica fillers) are already present in the silicone elastomer formulations. However, is difficult to determine the amount of resins as well as the type and modification of the fillers already embedded $^{2}$ in a commercial formulation and certainly beyond the scope of this article. The term "filler(s)" will therefore be used throughout the rest of this article to refer to both resins and inorganic fillers present in the silicone elastomers.

S186 formulations showed the highest thermal stability of all investigated silicone formulations, as can be seen from the degradation onset temperatures (Table 2). S186 formulations also showed the highest residual weight at $900^{\circ} \mathrm{C}$. Soft formulations (Eco 30 and Eco 50) were observed to have similar thermal stability and similar residual weight at $900^{\circ} \mathrm{C}$. Both thermal stability and residual weight of soft formulations (Eco 30 and Eco 50) were significantly lower compared to hard formulations (S184 and S186). In the case of MIX Eco 30_S184_3:1, blending of soft and hard segments was observed to decrease thermal stability compared to the respective soft and hard formulations in the blend, as onset temperature of degradation shifted towards lower values. This is most likely caused by the presence of further unreacted groups due to nonstoichiometric crosslinking reaction conditions, as discussed above. The presence of unreacted vinyl has previously been shown to decrease thermal stability ${ }^{\left[{ }^{1}\right]}$ However, the residual weights of blends at $900^{\circ} \mathrm{C}$ resulted in intermediate values, as expected from the combination of formulations with relatively low and high filler contents. Ogliani et al. ${ }^{[1]}$ reported data from thermogravimetric analysis under nitrogen of pure silicone networks resulting from $\mathrm{Pt}$ catalyzed silylation reactions with different crosslinking densities and showed that the residual weight of pure silicone networks at $700^{\circ} \mathrm{C}$ is $0 \%$. Due to the lack of available data on the chemistry of fillers and additives in the formulations investigated here, we assume that the residue of formulations at $900^{\circ} \mathrm{C}$ corresponds to the amount of filler in the formulation.

The hard Sylgard 184 formulation has low viscosity and contains fillers which maintain transparency, while the hard Sylgard 186 formulation and soft formulations (Ecoflex 00-30 and Ecoflex 00-50) have high viscosities and contain relatively high amounts of fillers, affecting transparency and causing their characteristic opacity. As discussed in the Introduction, if optical transparency or low viscosity is needed for a given application, resins are usually added rather than silica. It can therefore be concluded that the Sylgard 184 formulation contains a higher amount of resin compared to Sylgard 186, Ecoflex 00-30, and Ecoflex 00-50 formulations. Data presented in Table 2 shows that, as expected, the hard formulations contain a higher amount of filler than the soft formulations. It was observed that S186 formulations contained higher amounts of filler compared to S184 formulations. The amount of filler is higher in the case of S186_5:1 compared to S186_10:1, indicating that part B of the formulation contains more filler than part A. In the case of S184 formulations, the amount of filler in S184_5:1 is lower compared to S184_10:1, indicating that part A of the formulation has more 
filler than part B. Furthermore, Eco 30 has a slightly lower amount of filler compared to Eco 50. Our results are in contrast with previous studies indicating that Ecoflex is an unfilled silicone rubber. ${ }^{41}$ The blends (MIX Eco 30_S184_3:1 and MIX Eco 50_S184_3:1) were observed to have intermediate filler contents compared to the respective soft and hard formulations used.

Table 2. Analysis of the gel fraction and TGA data of commercial silicone formulations.

\begin{tabular}{|c|c|c|c|c|}
\hline Sample name & $\mathbf{w}_{\mathrm{gel}^{*}}$ & $\begin{array}{l}\text { Onset degradation } \\
\qquad\left[{ }^{\circ} \mathrm{C}\right]^{* *} \\
\end{array}$ & $\begin{array}{c}\text { Residual weight at } 900^{\circ} \mathrm{C} \\
{[\%]^{* *}}\end{array}$ & $\begin{array}{l}\text { Amount of filler } \\
{[\%]^{* * *}}\end{array}$ \\
\hline S184_5:1 & 0.95 & 373 & 46.1 & 46 \\
\hline S184_10:1 & 0.95 & 380 & 54.7 & 54 \\
\hline S186_5:1 & 0.96 & 415 & 68.3 & 68 \\
\hline S186_10:1 & 0.96 & 428 & 62.3 & 62 \\
\hline Eco 30 & 0.44 & 308 & 30.2 & 30 \\
\hline Eco 50 & 0.52 & 301 & 31.9 & 31 \\
\hline MIX Eco 30_S184_3:1 & 0.58 & 297 & 40.3 & 40 \\
\hline MIX Eco 50_S184_3:1 & 0.56 & 312 & 44.6 & 44 \\
\hline
\end{tabular}

*Experimentally determined weight fraction of gel. **Values calculated as an average value of data from three TGA measurements. ***Amount of filler is estimated to be equal to the residual weight at $900^{\circ} \mathrm{C}$.

\section{- Question 2: Are resins also inducing such effect?}

Response: We have made changes in the manuscript to indicate presence of resins in the tested commercial formulations. Since all tested formulations most likely have both resins and inorganic fillers embedded in the network, we have not discussed if and how significant would the Mullins effect be if only resins were present in the system.

Addition in the article on page 3, section -Introduction-: However, when resins are present, additional covalent bonds are introduced into the network (as compared to non-covalent interactions with, e.g. silica) and the polymer network formed has a higher crosslinking density than that of networks with only silica fillers present in the same concentration.

Addition in the article on page 5, section -Materials and sample preparation-: Since resins and inorganic fillers (most commonly silica fillers) are already present in silicone elastomer formulations, the elastomers are prepared as received from the supplier, without introduction of additional fillers.

Addition in the article on page 10, section -Analysis of the chemical characteristics of the elastomers-: As indicated above, resins and inorganic fillers (most commonly silica fillers) are already present in the silicone elastomer formulations. However, is difficult to determine the amount of resins as well as the type and modification of the fillers already embedded $^{2}$ in a commercial formulation and certainly beyond the scope of this article. The term "filler(s)" will therefore be used throughout the rest of this article to refer to both resins and inorganic fillers present in the silicone elastomers.

- Question 3: How reversible it is? 


\section{Response: We have used the stress energy function to quantify the softening effect in different formulations, and in that way also introduce a measure of reversibility of the sample geometry.}

Addition in the article on page 18, section -Cyclic uniaxial tension tests-: The strain energy function of the $1^{\text {st }}$ test can be used to quantify the Mullins effect in the different commercial formulations. Since the largest energy dissipation occurs in every first cycle in the strain rise path of the $1^{\text {st }}$ test, the dissipated energy, normalized by the total energy in every first cycle, can be used as a measure of the softening effect at different strains. Different formulations can be compared via the softening effect at identical applied strains. However, this comparison between different formulations at the same applied strain may lead to incorrect conclusions, since the stresses developed in the elastomer will be very different at an absolute strain, of e.g., 20\%. For example, the applied strain in the first cycle of the $1^{\text {st }}$ test is $20 \%$, which, in the case of hard formulations (S184 and S186), is 5 and 17 times lower, respectively, than the maximum strain that these formulations can sustain without breaking, but is 34 times lower in the case of soft formulations.

Figure 7 presents the dissipated energy normalized by the total energy for different formulations in the first cycle of the $1^{\text {st }}$ test at a strain of approximately $20 \%$ of $\varepsilon_{\mathrm{T}}$ for each formulation. This measure enables comparison of the softening effect in formulations at the same strain level relative to $\varepsilon_{\mathrm{T}}$. Data presented in Figure 7 shows that hard formulation S184_10:1 has a lower softening effect compared to S186_10:1, likely due to its lower filler content. The softening effect also decreased in S184_5:1 and S186_5:1 compared to S184_10:1 and S186_10:1, respectively. This decrease is as expected for S184_5:1, which contains less filler than S184_10:1. However, S186_5:1 has a higher filler content than S186_10:1; yet both S184_5:1 and S186_5:1 formulations contain higher percentege of part B compared to S184_10:1 and S186_10:1. Since part B of the elastomer formulations contains functionalized polymer chains as crosslinker, formulations with a 5:1 ratio of A and B parts contain a larger number of crosslinking points compared to formulations with 10:1. These results suggest that a highly crosslinked network with higher concentration of crosslinking points experiences less reorganisation and less sliding of fillers, as well as less chain slippage, resulting in a reduced softening effect compared to networks with lower concentrations of crosslinking points. Unsurprisingly, then, Eco 50 and blends (MIX Eco 30_S184_3:1 and MIX Eco 50_S184_3:1) exhibit a lower softening effect compared to hard formulations. SEC data indicates that all formulations consist of both high and low molecular weight polymer chains. However, soft formulations' polymer chains have significantly higher molecular weights compared to those of hard formulations. This is also expected, since elasticity scales with crosslinking density, which again scales inversely to the molecular weight of the polymer. TGA data also show that the soft formulations contain less filler compared to hard formulations, suggesting that this lower filler content is one reason for the lower softening effect in soft formulations compared to hard. The synergetic effect of long and short polymer chains in the soft formulations may also contribute to soft formulations and blends' comparatively reduced softening effect in relation to purely hard formulations. ${ }^{[58]}$ Both long and short polymer chains in soft formulations are significantly longer compared to those in hard formulations. The unexpectedly high softening effect observed in case of Eco 30 compared to all other formulations may be a result of the relatively high sol fraction in Eco 30 films. All polymer chains are initially stretched under low applied strain, contributing to overall stress, ${ }^{[58]}$ upon increased strain, however, the sol fraction polymer chains will relax their stresses and not contribute further to the overall stress. At 120\% strain and below, Eco 30 displays a higher softening effect than Eco 50. However, this situation reverses at higher applied strains (See Figure 47S. in SI). Blends MIX Eco 30_S184 and MIX Eco 50_S184 have lower softening effect compared to the soft 
formulations (Eco 30 and Eco 50). Results show that blending increases the reversibility of the sample geometry compared to both soft and hard formulations used in the blend, as expected due to the increased crosslinker content compared to the soft formulations.

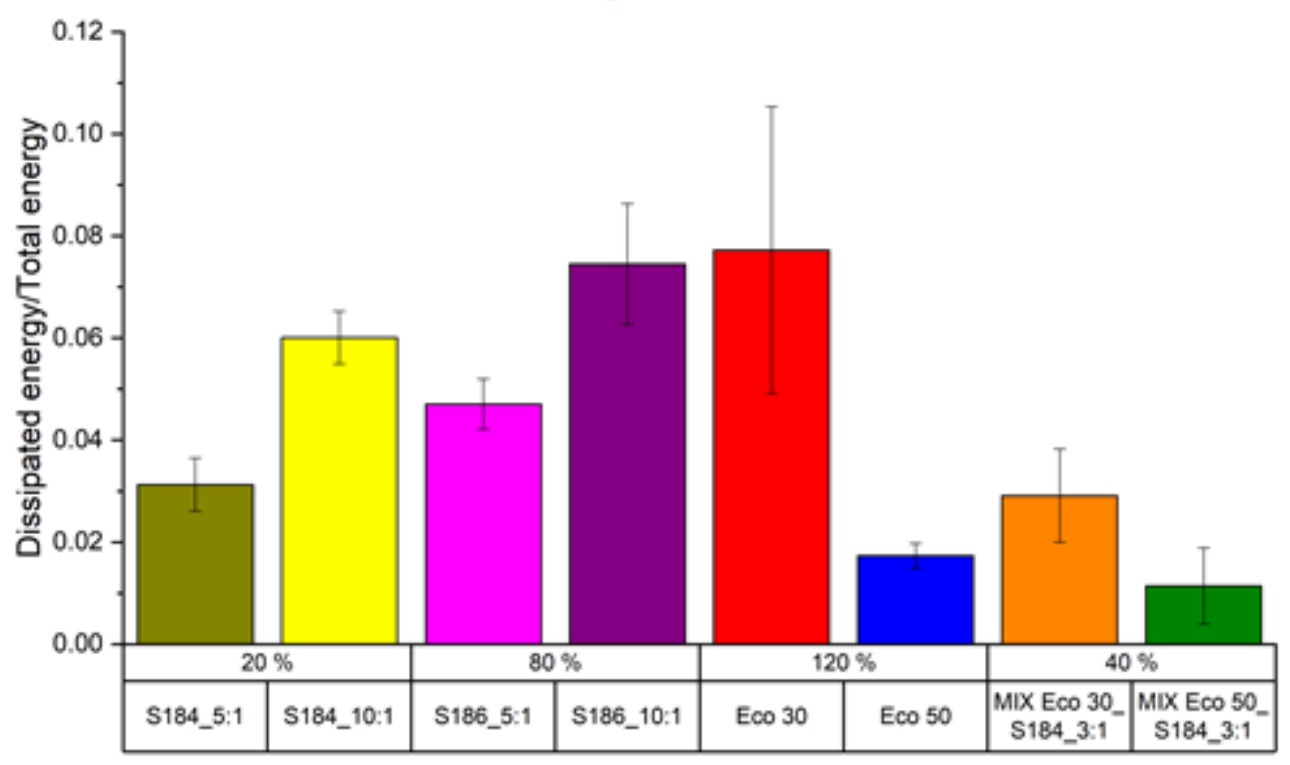

Figure 7. Dissipated energy normalized by the total energy of different silicone formulations in the first cycle of the $1^{\text {st }}$ test at approximately $20 \%$ of strain at break.

\section{- Question 4: How one can fight against it?}

Response: We have made changes in the manuscript to address the question.

Additions in the article on page 3, section -Introduction-: Without a comprehensive theory to rely on, then how and where, do we observe the Mullins effect in practical terms? A common way to make thin elastomers is to coat the liquid silicone elastomer mixture onto a substrate e.g., a plastic film or glass plate - and then cure the elastomer on the substrate. The elastomer is then released from the substrate after curing, but, since the adhesion forces are relatively large compared to the elastic forces of the thin silicone elastomer, it undergoes stretching that is often beyond the elastic regime of its particle-particle interactions. The mechanical properties of the elastomer may already have experienced the Mullins effect by this stretch, and in this way its material properties become sensitive to processing conditions. Elastomers are typically observed to exhibit their highest Young's modulus in the first deformation cycle; when reporting data or designing products, one must therefore be aware that the first cycle at, e.g., constant stress, will give lower strain response than the subsequent steady-state cycles. ${ }^{[19]}$

Reversible stretchability and flexibility render silicone elastomers suitable materials for soft transducers ${ }^{[32-34]}$, soft robots ${ }^{[35]}$, medical devices ${ }^{[32,36]}$, and many other futuristic applications. Silicone elastomers used for different applications are expected to maintain reliable properties and performance under long-term employment. Accounting for the Mullins effect in silicone formulations offers a simple means of avoiding experimental discrepancies and product unreliability.

Addition in the article on page 18, section - Cyclic uniaxial tension tests -: This indicates that the largest energy dissipation in the $2^{\text {nd }}$ test occurs in the first cycle. It is therefore clear that the silicone elastomer formulations exhibit idealized Mullins behavior after the first cycle in the $2^{\text {nd }}$ test, since the softening effect is negligible in all subsequent deformation cycles. ${ }^{[20]}$ This suggests that the softening effect can be avoided in silicone elastomer forumulations by prestretching the sample to $\varepsilon_{\max }$ of the particular formulation (or, alternatively, beyound the 
maximum strain envisioned for the product) one time before the elastomer is employed at the given product.

Addition in the article on page 20, section - Cyclic uniaxial tension tests -: However, in contrast to the common understanding that the Mullins effect increases with increasing filler content (hardness), our results show that the Mullins effect actually becomes more prominent when the elastomer network becomes very soft. This indicates that the Mullins effect is not only impacted by the presence of fillers, but also by the number of bonded species in the network (gel fraction), number of crosslinking points, and the molecular weight of polymer chains. This further suggests that the softening effect may be limited by choosing a silicone elastomer formulation with low filler content, high molecular weight polymer chains, and a high number of crosslinking functionalities, thereby ensuring that a network with low sol fraction is obtained.

- Question 5: Is the type of modification of the filler relevant?

Response: We made changes in the manuscript to indicate presence of the fillers. However, since the type and possible modification of the filler in formulations are not known, conclusions regarding influence of the filler modification on Mullins effect was not discussed. However, changes in the article that we made, and are somewhat related to this question are indicated in the answer to the above Question 1: What type of filler induces the worst Mullins effect?

\section{Reviewer \#2:}

- Comments: There is a very large literature on the characterization and modeling of the Mullins softening, that includes, although less abundant, silicone rubbers. The authors do not seem up to date on the bibliography. The paper is more like a characterization of the Mullins softening of their silicon material which does not bring anything new general knowledge. The problematic for robots might be of importance, although Mullins softening is evacuated on first load and is not recoverable, except in particular conditions of temperatures. There is not enough new content in terms of research on the mechanical behavior of filled silicon to recommend for publication.

> Zhong et al. (2019), A physically-based damage model for soft elastomeric materials with anisotropic Mullins effect, International Journal of Solids and Structures, 176-177, 121-134.

> Rebouah et al. (2015) Development and modeling of filled silicone architectured membranes, meccanica, 50, 11-24.

> Rebouah et al. (2013) Anisotropic modeling of the Mullins effect and the residual strain of filled silicone rubber, Constitutive models for rubber VIII, ECCMR 2013, 111-116.

> Merckel et al. (2013) Constitutive modeling of the anisotropic behavior of Mullins softened filled rubbers. Mechanics of Materials, 57, 30-41.

> Merckel et al. (2012) A Mullins softening criterion for general loading conditions. Journal of the Mechanics and physics of solids, 60, 1257-1260. 
D Diaz et al. (2014), Physical interpretation of the Mullins softening in a carbonblack filled

SBR,

Polymer,

55 ,

$4942-4947$

Response: Thank you for your comments and suggesting literature which is relevant for the topic. We have included some of the suggested articles in our manuscript. However, some of the articles are related to the modeling of the Mullins effect, while in this work we decided to focus solely on experimental investigation of Mullins effect in commercial silicone elastomer formulations. We have also included detailed characterization of the commercial silicone elastomers used in this work and made a correlation between Mullins effect and different silicone elastomers.

\section{Addition in the article on page 6 , section - Measurements -:}

3.1. Fourier Transform Infrared Spectroscopy Analysis

Fourier transform infrared (FTIR) spectroscopy is carried out using a Nicolet iS50 FTIR spectrometer (Thermo Fisher Scientific, USA). Wavenumbers between 400 and $4000 \mathrm{~cm}-1$ are recorded with 64 interferograms for each sample formulation as well as their respective A and B parts, in transmission mode.

\subsection{Extraction measurements}

The gel fractions are determined using gel extraction, in which small pieces of the sample film $(\sim 200 \mathrm{mg}$ ) are immersed in chloroform $(\sim 10 \mathrm{~mL})$ for $48 \mathrm{~h}$ at room temperature (approximately $23^{\circ} \mathrm{C}$ ). The solvent is replaced after $24 \mathrm{~h}$; after $48 \mathrm{~h}$, the solvent is poured out and the films are washed twice with fresh solvent. The samples are then dried for $48 \mathrm{~h}$ at room temperature under ambient pressure, until constant weight is obtained. Gel fractions are calculated as $\mathrm{wgel}_{\mathrm{g}}$ $=\mathrm{m}_{\mathrm{e}} / \mathrm{m}_{0}$, where $\mathrm{m}_{\mathrm{e}}$ is the weight after extraction and drying, and $\mathrm{m}_{0}$ is the initial weight of the sample..$^{[44]}$

\subsection{Thermogravimetry analysis}

Thermogravimetry analysis (TGA) of the sample films is performed on a thermogravimetric analyzer Discovery series (TA instruments). Samples $(\sim 10 \mathrm{mg})$ are heated from room temperature to $900^{\circ} \mathrm{C}$ in a platinum pan under a nitrogen flow $(25 \mathrm{~mL}$ min-1) at a heating rate of $10^{\circ} \mathrm{C}$ min-1. Measurements are repeated 3 times.

\subsection{Nuclear magnetic resonance spectroscopy}

Nuclear magnetic resonance (NMR) spectroscopy is performed on a Bruker $300 \mathrm{MHz}$ spectrometer. A and B parts of Ecoflex 00-30 and Ecoflex 00-50 formulations (approximately $50 \mathrm{mg})$ are dissolved in $0.7 \mathrm{~mL}$ of chloroform-d $(\mathrm{CDCl} 3)$. Data are analyzed using TopSpin version $3.5 \mathrm{pl} 7$ from Bruker.

\subsection{Size exclusion chromatography}

Size exclusion chromatography (SEC) is performed on a chromatographic system consisting of a PL-ELS 2100 module connected to a Viscotek TriSEC model 302 triple detector array (RI, light scattering, viscometer). The PL Guard and two PL gel mixed D columns from Polymer Laboratories are connected in series, providing resolution up to 300,000 Daltons. The system is calibrated using linear polydimethylsiloxane (PDMS) standards acquired from PSS (Mainz, Germany). SEC measurements of A and B parts of soft and hard formulations are performed. Both A and B parts of soft formulations are first dissolved in toluene and filtered through Celite (Celite ${ }^{\circledR} 545$, Merck KGaA) and filter paper (VWR), retaining particles 5-13 $\mu \mathrm{m}$ in size. A and B parts of soft and hard formulations (approximately $2.5 \mathrm{mg}$ ) are dissolved in $1 \mathrm{~mL}$ of toluene and again filtered through a filter syringe with $0.45 \mu \mathrm{m}$ pore size. Samples are run at a 
room temperature $\left(23^{\circ} \mathrm{C}\right)$ in tetrahydrofuran $(\mathrm{THF})$ as mobile phase, with a flow rate of $1 \mathrm{~mL}$ $\min ^{-1}$. Molar mass characteristics are calculated using OmniSEC software.

Addition in the article on page 8, section -Results and Discussion-: Below, the respective formulations will be extensively investigated in order to obtain further details regarding the chemical structure of the resulting networks. This investigation is not a full characterization of the network structure, however, since silicone elastomers are complex mixtures of polymers and crosslinkers, and potentially also inorganic fillers, resins, chain extenders, and additives.

\subsection{Analysis of the chemical characteristics of the elastomers}

FTIR was used to analyze molecular structure and functional groups in the prepared silicone films. FTIR analysis of A and B parts of each formulation was also performed. The FTIR results for the S184_10:1 as well as A and B parts of the Sylgard 184 formulation are shown in Figure 2. While A and B parts of Sylgard 184 and S184_10:1 serve as an example, all formulations investigated in this study showed similar behavior (Data in SI).

The transmittance peaks at $2960 \mathrm{~cm}^{-1}$ were attributed to the $\mathrm{C}-\mathrm{H}$ stretching vibration of the methyl group $\left(-\mathrm{CH}_{3}\right)$. The peaks at $1460 \mathrm{~cm}^{-1}$ were attributed to the $\mathrm{C}=\mathrm{C}$ deformation of $\mathrm{Si}-$ $\mathrm{CH}=\mathrm{CH}_{2}$. Both $\mathrm{A}$ and $\mathrm{B}$ parts of the formulation contain PDMS with vinyl functionalities. Furthermore, the presence of the peaks at $1460 \mathrm{~cm}^{-1}$ in the case of S184_10:1 indicates that not all vinyl groups reacted in the hydrosilylation reaction. The peaks at $1255 \mathrm{~cm}^{-1}$ were attributed to the symmetric deformation of $\mathrm{Si}-\mathrm{CH}_{3}$. The strong transmittance peaks at $1000-$ $1100 \mathrm{~cm}^{-1}$ were attributed to the stretching vibrations of $\mathrm{Si}-\mathrm{O}-\mathrm{Si}$. The strong peaks at $800 \mathrm{~cm}^{-1}$ were attributed to the $\mathrm{Si}-\mathrm{C}$ stretching vibrations of $\mathrm{Si}-\left(\mathrm{CH}_{3}\right)_{2}$. All these peaks are characteristic of the PDMS. In the case of part B of Sylgard 184, the distinctive transmittance peaks at 2158 $\mathrm{cm}^{-1}$ and $910 \mathrm{~cm}^{-1}$ were attributed to $\mathrm{Si}-\mathrm{H}$ vibrations, indicating that part $\mathrm{B}$ also contains PDMS with hydride functionality. The disappearance of these $\mathrm{Si}-\mathrm{H}$ peaks in the case of S184_10:1 indicates that the hydrosilylation reaction was successfully finished. The presence of the $\mathrm{C}=\mathrm{C}$ peaks and disappearance of the $\mathrm{Si}-\mathrm{H}$ peaks in the case of S184_10:1 indicates an excess of vinyl groups in the formulation.

Peaks at $2158 \mathrm{~cm}^{-1}$ and $910 \mathrm{~cm}^{-1}$ were also observed in the case of part B of the other hard formulation, Sylgard 186 (Data in SI). In case of part B of soft formulations (Ecoflex 00-30 and Ecoflex 00-50), however, the peak at $2158 \mathrm{~cm}^{-1}$ was not observed, while the intensity of the peak at $910 \mathrm{~cm}^{-1}$ was quite low (Data in SI). This indicates a low concentration of hydride groups (below the instrument's detection limit) in the soft formulations, a finding that is in good agreement with their low crosslinking densities.

The amount of bonded (gel fraction) species in the networks was determined from swelling experiments with chloroform, the results of which are presented in Table 2. In general, the gel fraction was found to be above approximately $95 \% \mathrm{w} / \mathrm{w}$ for all hard formulation films, and above approximately $52 \% \mathrm{w} / \mathrm{w}$ for the Eco 50 film. However, the gel fraction was found to be less than $44 \% \mathrm{w} / \mathrm{w}$ for the Eco 30 film. These findings are in good agreement with the elastomers' properties as claimed on their respective data sheets (to be elucidated further in this article). The gel fractions of the blends (MIX Eco 30_S184_3:1 and MIX Eco 50_S184_3:1) were also found to be higher compared to the respective soft formulations (Eco 30 and Eco 50). However, the gel fraction of MIX Eco 50_S184_3:1 film was approximately $7.7 \%$ (relatively) higher than the soft formulation Eco 50 film, while the gel fraction of MIX Eco 30_S184_3:1 was approximately 31\% (relatively) higher than the soft formulation Eco 30. These results suggest that blending soft and hard segments, as in the case of MIX Eco 50_S184_3:1, can induce a small inhibitory effect on the crosslinking efficiency of the two formulations in the blend. This is not surprising, however, since the crosslinking of the blends was not optimized in these studies. 
Thermogravimetric analysis of cured silicone formulations was performed from room temperature to $900^{\circ} \mathrm{C}$ under nitrogen atmosphere to inspect general thermal decomposition behavior of the cured silicone elastomer formulations and obtain information on the solid residue after thermal treatment. The onset of thermal degradation is commonly predicted using the temperature at which $5 \%$ weight loss is recorded. ${ }^{[4-47]}$ Onset temperatures of degradation, residual weights at $900^{\circ} \mathrm{C}$, and amounts of filler in each formulation are presented in Table 2. As indicated above, resins and inorganic fillers (most commonly silica fillers) are already present in the silicone elastomer formulations. However, is difficult to determine the amount of resins as well as the type and modification of the fillers already embedded ${ }^{2}$ in a commercial formulation and certainly beyond the scope of this article. The term "filler(s)" will therefore be used throughout the rest of this article to refer to both resins and inorganic fillers present in the silicone elastomers.

S186 formulations showed the highest thermal stability of all investigated silicone formulations, as can be seen from the degradation onset temperatures (Table 2). S186 formulations also showed the highest residual weight at $900^{\circ} \mathrm{C}$. Soft formulations (Eco 30 and Eco 50) were observed to have similar thermal stability and similar residual weight at $900^{\circ} \mathrm{C}$. Both thermal stability and residual weight of soft formulations (Eco 30 and Eco 50) were significantly lower compared to hard formulations (S184 and S186). In the case of MIX Eco 30_S184_3:1, blending of soft and hard segments was observed to decrease thermal stability compared to the respective soft and hard formulations in the blend, as onset temperature of degradation shifted towards lower values. This is most likely caused by the presence of further unreacted groups due to nonstoichiometric crosslinking reaction conditions, as discussed above. The presence of unreacted vinyl has previously been shown to decrease thermal stability. ${ }^{[1]}$ However, the residual weights of blends at $900^{\circ} \mathrm{C}$ resulted in intermediate values, as expected from the combination of formulations with relatively low and high filler contents. Ogliani et al. ${ }^{[1]}$ reported data from thermogravimetric analysis under nitrogen of pure silicone networks resulting from Pt catalyzed silylation reactions with different crosslinking densities and showed that the residual weight of pure silicone networks at $700^{\circ} \mathrm{C}$ is $0 \%$. Due to the lack of available data on the chemistry of fillers and additives in the formulations investigated here, we assume that the residue of formulations at $900^{\circ} \mathrm{C}$ corresponds to the amount of filler in the formulation.

The hard Sylgard 184 formulation has low viscosity and contains fillers which maintain transparency, while the hard Sylgard 186 formulation and soft formulations (Ecoflex 00-30 and Ecoflex 00-50) have high viscosities and contain relatively high amounts of fillers, affecting transparency and causing their characteristic opacity. As discussed in the Introduction, if optical transparency or low viscosity is needed for a given application, resins are usually added rather than silica. It can therefore be concluded that the Sylgard 184 formulation contains a higher amount of resin compared to Sylgard 186, Ecoflex 00-30, and Ecoflex 00-50 formulations. Data presented in Table 2 shows that, as expected, the hard formulations contain a higher amount of filler than the soft formulations. It was observed that S186 formulations contained higher amounts of filler compared to S184 formulations. The amount of filler is higher in the case of S186_5:1 compared to S186_10:1, indicating that part B of the formulation contains more filler than part A. In the case of S184 formulations, the amount of filler in S184_5:1 is lower compared to S184_10:1, indicating that part A of the formulation has more filler than part B. Furthermore, Eco 30 has a slightly lower amount of filler compared to Eco 50. Our results are in contrast with previous studies indicating that Ecoflex is an unfilled silicone rubber. ${ }^{41}$ The blends (MIX Eco 30_S184_3:1 and MIX Eco 50_S184_3:1) were observed to have intermediate filler contents compared to the respective soft and hard formulations used. 
A common rule when formulating silicone elastomers is that the Pt catalyst should be kept separate from any hydride functional oligomers and polymers, since the Pt may facilitate condensation of the hydride group over time. One premix therefore usually contains the polymer (vinyl functional) and the catalyst, while the other contains crosslinker mixed with polymer (vinyl functional). The other constituents are mixed into the respective parts depending on their functionalities and their compatibility with Pt and hydride. The A part of all investigated elastomer formulations in this study can easily be shown to contain the catalyst and polymer, with the B part containing hydride functional crosslinker and polymer, as confirmed by a simple experiment in which a drop of catalyst and a drop of crosslinker, respectively, were added to the two parts. A parts became solid upon the addition of crosslinker, but not upon addition of catalyst, while B parts behaved oppositely.

1H NMR spectra of A and B parts of Ecoflex 00-30 are presented in Figure 3. 1H NMR spectra of $\mathrm{A}$ and B parts of Ecoflex 00-50 are similar and are presented in Figure $2 \mathrm{~S}$ in the SI. CDCl3 was used as the solvent for NMR measurements and resulted in a strong signal at $7.25 \mathrm{ppm}$. Methylsilyl groups exhibited strong signals between $-0.25 \mathrm{ppm}$ and $0.5 \mathrm{ppm}$. Signals between $5.68 \mathrm{ppm}$ and $6.19 \mathrm{ppm}$ were assigned to vinyl groups, while signals at $0.88 \mathrm{ppm}$ and $1.21 \mathrm{ppm}$ were assigned to geminate silanol and isolated silanol, respectively. ${ }^{[2]}$ Broad signal at $1.64 \mathrm{ppm}$ was assigned to water, which is most likely introduced via the solvent. ${ }^{[48]}$

Even though FTIR spectra of A and B parts of Ecoflex 00-30 did not indicate the presence of the characteristic hydride peak, 1H NMR spectroscopy revealed that part B contains hydride functionalities, as can be seen from the strong peak at $4.72 \mathrm{ppm}$. This suggests that Ecoflex formulations are likely cross-linked by hydride addition to vinyl groups in the presence of platinum catalyst ${ }^{49,50]}$. However, the NMR analysis can not verify if there is more than one type of crosslinker, or potentially chain extenders (small, linear polymers with terminal hydride functionalities) as well.

1H NMR spectra of extracts of cured Eco 30 and Eco 50 films showed clear hydride peaks, indicating that not all hydride functions reacted (Figure S3 in SI). This result agrees with extraction experiments, which showed low gel fraction in the films. NMR spectra of Eco 30 and Eco 50 extracts also showed peaks which most likely originate from the Pt-catalyst; this was confirmed by $1 \mathrm{H}$ NMR of the Karstedt's catalyst (Figure S3 in SI).

A and B parts of hard formulations Sylgard 184 and Sylgard 186, as well as of soft formulations Ecoflex 00-30 and Ecoflex 00-50, were analyzed by size exclusion chromatography to assess the apparent molecular mass, polydispersity, weight fractions of long chains in each part (A and B) of the formulations, and overall weight fraction of long chains in hard and soft formulations as a whole. These distributions give an indication of the formulation strategy used. Chromatograms of A and B parts of all formulations showed bimodal distributions. A summary of results for all A and B parts is shown in Table 3, along with interpretation of the resulting weight fraction of long polymer chains in the final elastomer mixtures.

Data indicates that both parts of all investigated compositions consist of both long (high molecular weight) and short (low molecular weight) polymer chains. The results can be interpreted as the Sylgard formulations are relatively closely related, with the vinyl functional polymers having a molecular weight in the range of $10-30 \mathrm{~kg} / \mathrm{mol}$ and being crosslinked by low molecular weight crosslinkers, potentially also with chain extenders. The softer of these two formulations (Sylgard 186) is characterized by longer vinyl functional polymers. The low molecular weight species in part A may be a low molecular weight vinyl functional polymer, which produces better properties due to the bimodality of the resulting networks. ${ }^{[51]}$ The Ecoflex formulations show higher molecular weights across all components. The relatively low fractions of long polymer ( $\mathrm{W}_{\mathrm{f} \text { long chains }}$ ) indicate a very different formulation strategy from that of the Sylgard formulations. However, their inherent softness indicates a long distance between crosslinking sites, so either they use chain extenders and/or, as indicated by NMR/FTIR, 
incomplete crosslinking due to nonstoichiometric ratios between hydride and vinyl functional constituents. Summarized findings of different properties of characterized silicone elastomers are presented in Table 4.

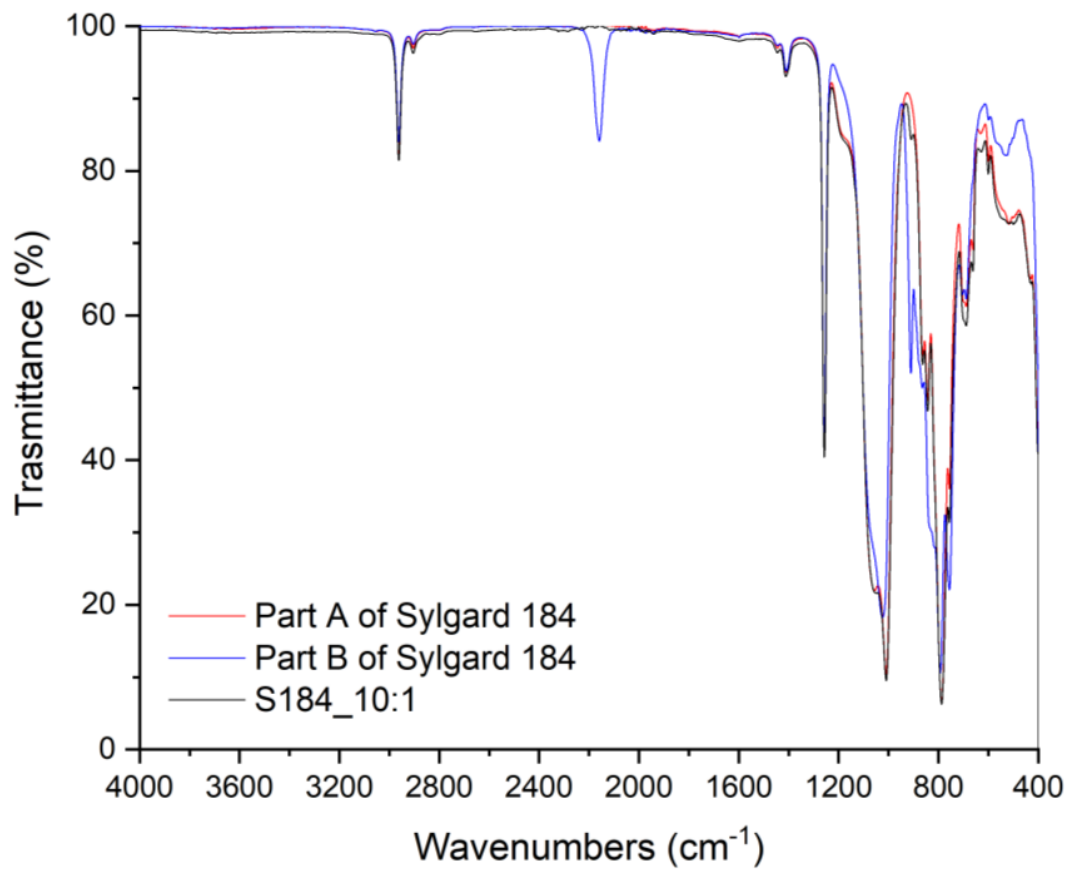

Figure 2. FTIR spectra of part A (red) and part B (blue) of Sylgard 184 formulation, and the final elastomer S184_10:1 sample (black).

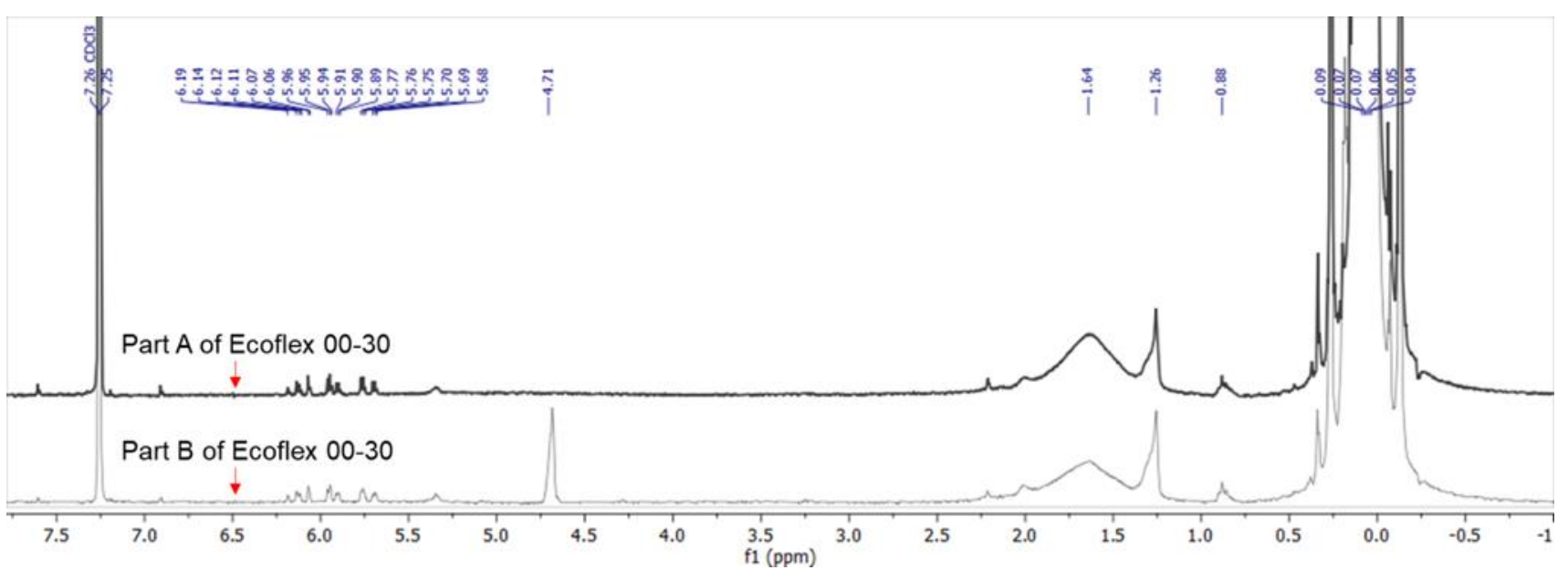

Figure 3. 1H NMR spectra of part A (black line) and part B (grey line) of Ecoflex 00-30.

Table 2. Analysis of the gel fraction and TGA data of commercial silicone formulations.

\begin{tabular}{|c|c|c|c|c|}
\hline Sample name & $\mathbf{w}_{\mathrm{gel}}{ }^{\star}$ & $\begin{array}{l}\text { Onset degradation } \\
{\left[\left[{ }^{\circ} \mathrm{C}\right]^{* *}\right.} \\
\end{array}$ & $\begin{array}{c}\text { Residual weight at } 900^{\circ} \mathrm{C} \\
{[\%]^{* *}}\end{array}$ & $\begin{array}{c}\text { Amount of filler } \\
{[\%]^{* * *}}\end{array}$ \\
\hline S184_5:1 & 0.95 & 373 & 46.1 & 46 \\
\hline S184_10:1 & 0.95 & 380 & 54.7 & 54 \\
\hline S186_5:1 & 0.96 & 415 & 68.3 & 68 \\
\hline S186_10:1 & 0.96 & 428 & 62.3 & 62 \\
\hline Eco 30 & 0.44 & 308 & 30.2 & 30 \\
\hline Eco 50 & 0.52 & 301 & 31.9 & 31 \\
\hline MIX Eco 30_S184_3:1 & 0.58 & 297 & 40.3 & 40 \\
\hline
\end{tabular}


*Experimentally determined weight fraction of gel. **Values calculated as an average value of data from three TGA measurements. ***Amount of filler is estimated to be equal to the residual weight at $900^{\circ} \mathrm{C}$.

Table 3. SEC data of commercial silicone formulations. All samples show a bimodal distribution and thus a pair of number average and weight average molecular weights is given for all samples along with overall average molecular weights $\mathrm{M}_{\mathrm{n} \text {-tot }}$ and $\mathrm{M}_{\mathrm{w} \text {-tot. }}$ The wt $\%$ of the high molecular weight parts is stated as $\mathrm{W}_{\text {long, }}$, and overall wt \% of high molecular weight parts in the hard and soft formulations is stated as $\mathrm{W}_{\mathrm{f} \text { long chains. }}$

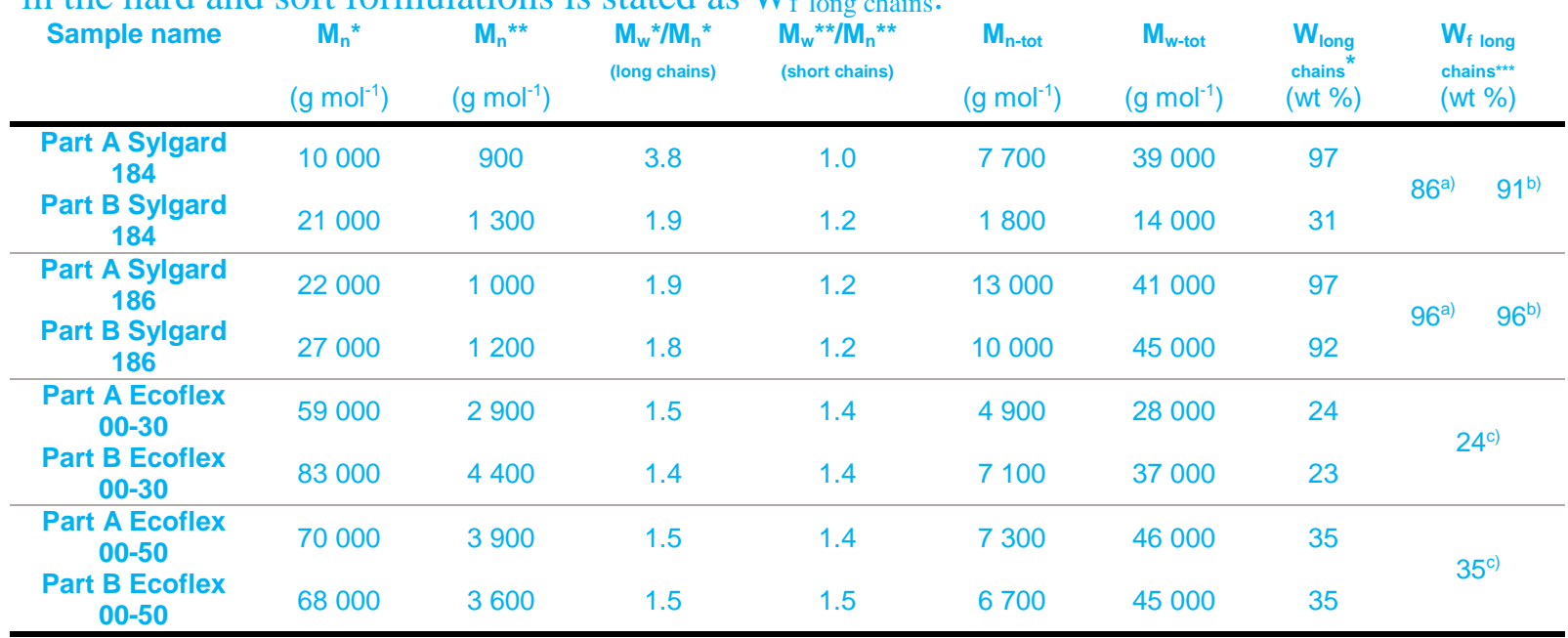

*Values determined from the first peak of size exclusion chromatography. **Values determined from the second peak of size exclusion chromatography. $* * *$ Values determined by accounting for mixing ratios of $\mathrm{A}$ and $\mathrm{B}$ parts in hard and soft formulations: a) ratio of $\mathrm{A}$ and B parts in hard formulations $5: 1, b$ ) ratio of $A$ and $B$ parts in hard formulations 10:1, and c) ratio of $\mathrm{A}$ and $\mathrm{B}$ parts in soft formulations 1:1.

As well as the addition on page 18, section -Cyclic uniaxial tension tests- which is shown in the answer to the Question 3 asked by Reviewer \#1. How reversible it is?

Other remarks: Page 3 starting line 7: Beware, the particles do not induce a plastic response. The Mullins effect is the signature of damage not plasticity. The Mullins effect induces a non reversible softening due to damage. This is rather different then plasticity. Figures like figure 9 are difficult to read. I do not discuss the number of cycle which a fatigue problem beyond the Mullins softening. Please read the literature on fatigue of filled rubbers.

Response: Thank you for the advice. We have made corrections in the text, so there is no confusion between the Mullins effect and the plasticity.

Modification in the article on page 3, section -Introduction-: All these different interactions, together with the nano- and micro-scale damages within the elastomer, reduce the reversibility of the elastomer dimensions after subjection to a cyclic strain deformation: this is called the Mullins effect. ${ }^{[8,21-23]}$ In rheological terms, it means that the elastomer has a stress memory function, such that the stress at a given strain will depend on the strain history of the sample. Even though the Mullins effect has been observed in unfilled elastomer rubbers, this strain-induced phenomenon is more pronounced in filler-reinforced elastomers. ${ }^{[24]}$ 
Response: However, we have not changed the figure below (In the previous version Figure 9S, in the revised article Figure 19S), since we did not come up with a better representation containing all the required information. We are open for suggestions how to present the data in better and more intuitive way, and we will gladly make further corrections and changes if needed.

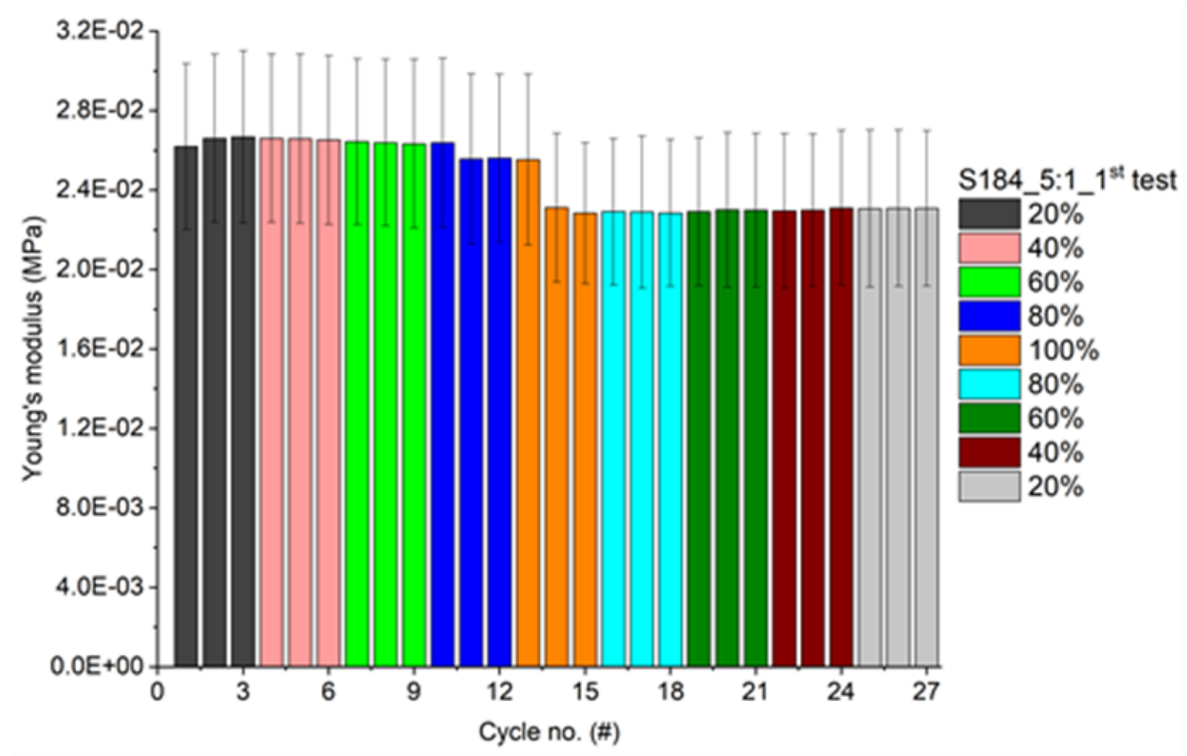

Figure 19S. Young's modulus (at 10\% strain) as a function of cycle number for different strains of S184_5:1 subjected to a $1^{\text {st }}$ test. After cycles 13-15, the strain path was reversed.

\section{Reviewer \#3}

Comments: Krpovic et al report on measurements of the Mullins effect on several commercial silicones. They present a systematic and thorough study, using uniaxial tensile tests, observing softening at every first cycle to higher strain. The experimental method is rigorous, and in line with how other groups have studied the Mullins effect. The intro could be strengthened by explaining to the reader why s/he should be concerned with Mullins effect in silicone elastomers, and what range of strain is relevant. Providing a few concrete examples where the Mullins effect is problematic would greatly help to motivate the study. The overall results are unsurprising, and roughly consistent with earlier experiments. I miss a discussion on how the data in this manuscript is different from data from other groups.

Response: Thank you very much for your comments.

- Question 1: Is testing method different?

Response: We have made changes in or manuscript to address the question.

Addition in the article on page 20, section -Cyclic uniaxial tension tests-: All mechanical test methods reported here are in agreement with previous studies ${ }^{[2,35,38,41,42,59-61]}$ on the 
Mullins effect in elastomers, with some differences. Previous studies used either rectangular shaped specimens ${ }^{[38,41,60,61]}$ or different dog bone shaped specimens ${ }^{[35,42,59]}$. These studies also reported different strain rates, number of cycles, and maximum applied strains, all of which affect results. Reported maximum applied strains of soft formulation samples (Eco 30 and Eco 50) in uniaxial cyclic tests are significantly lower than the maximum applied strains in this work. ${ }^{[38,41]}$ The higher extensibility of the dog bone shaped elastomer samples reported here is expected, since the dog bone geometry is used to eliminate high stress concentrations. Furthermore, previous studies did not report test methods containing both "strain rise" and "strain drop" paths. ${ }^{[35,38,41,59-61]}$

\section{Question 2: How do results compare?}

Response: Thank you for your question. Due to the differences in the testing conditions between our and other groups, which are indicated in the response to the previous question, we have not focused our scientific discussion on comparison of the results between our and other groups. We have to mention that our results are in line with other groups that have studied the Mullins effect. The stress-strain curves of tested silicone elastomers in cyclic uniaxial tension tests are same as in other reports. However, unlike other groups that performed modeling of Mullins effect and fitting of experimental data to proposed models, we focused our work solely on experimental investigation of Mullins effect. We use stress energy function to quantify the softening effect in different silicone formulations, which has not been reported previously.

Comments: I list just a few papers below. There are more. The authors should conduct a more through literature review and compare their data to put it in context.

- $\quad$ Liao, Z., Hossain, M. \& Yao, X. Ecoflex polymer of different Shore hardnesses: Experimental investigations and constitutive modelling. Mechanics of Materials 144, 103366 (2020). https://doi.org/10.1016/j.mechmat.2020.103366 Shows Mullins data on Ecoflex (like this manuscript)

- this paper that just came out, same material set: doi: 10.1016/j.polymertesting.2020.106478

- $\quad$ Rosset, S., Maffli, L., Houis, S. \& Shea, H. R. An instrument to obtain the correct biaxial hyperelastic parameters of silicones for accurate DEA modelling. Proc SPIE EAPAD 90560M (2014). doi:10.1117/12.2044777. -Shows Mullins data on Sylgard 186 (like this manuscript) and Nusil CF19

- Rey, T., Chagnon, G., Le Cam, J.-B. \& Favier, D. Influence of the temperature on the mechanical behaviour of filled and unfilled silicone rubbers. Polymer Testing 32, $492-501$ (2013). Doi. 10.1016/j.polymertesting.2013.01.008 Shows Mullins data Silicone Bluestar RTV 141 and RTV 3428 with fillers

- $\quad$ The tensile data from methods $1 \& 2$ are well presented, but the analysis is not quantitative between materials.

Response: Thank you for your comments and for suggesting literature which is relevant for the topic.

- Question 3: Can the strain-energy function be used to quantitatively compare the different silicones? 
Response: Thank you for your suggestion. We have used the strain-energy function to quantitatively compare different silicones formulations. The additions made in the article which refer to this question are indicated in the answer to the Question 3 asked by Reviewer \#1: How reversible it is?

- Question 4: Or can you come up with a metric to give a relative importance of the Mullins effect?

Response: As suggested with pervious question, and the reply to it, we have used the strain-energy function to give a relative importance of the Mullins effect.

- Question 5: What does the reader learn about how the different silicones exhibit different (or the same) magnitude of Mullins effect. Comment: This manuscript lacks a discussion on how Mullins effect is different for silicones compared to other elastomers.

Response: As indicated in the answers to the previous questions, we used strain energy function to quantitatively compare different silicones formulations. This analysis led to the conclusion that even though Mullins effect is more pronounced in the filler reinforced systems, there are also other parameters that have significant influence on the Mullins effect in the silicone elastomers.

Addition in the article on page 20, section -Cyclic uniaxial tension tests-: Results also show that the Mullins effect is indeed more pronounced in elastomers which contain higher amounts of filler ${ }^{[24]}$ (hard formulations). However, in contrast to the common understanding that the Mullins effect increases with increasing filler content (hardness), our results show that the Mullins effect actually becomes more prominent when the elastomer network becomes very soft. This indicates that the Mullins effect is not only impacted by the presence of fillers, but also by the number of bonded species in the network (gel fraction), number of crosslinking points, and the molecular weight of polymer chains. This further suggests that the softening effect may be limited by choosing a silicone elastomer formulation with low filler content, high molecular weight polymer chains, and a high number of crosslinking functionalities, thereby ensuring that a network with low sol fraction is obtained.

- Question 6: Do we expect order of magnitude differences? eg Chen, Y., Kang, G., Yuan, J. \& Li, T. Experimental study on pure-shear-like cyclic deformation of VHB 4910 dielectric elastomer. J Polym Res 26, 186 (2019). doi: 10.1007/s10965-019-1858-6 report data on acrylic VHB (indeed not a silicone, but relevant comparison). Or many other papers.

Response: Thank you for your question. We have not compared silicones with other elastomers, and therefore can not judge the order of magnitude difference. However, in our work, we compared different silicone elastomer formulations we tested, and as indicated in the answers to the previous questions, the strain energy function is used to quantify the softening effect in different formulations and determine the differences 
in softening effect between different formulations. Changes that we made are already indicated in the answer to the Question 3 asked by Reviewer \#1: How reversible it is?

- Question 7: If I want to avoid the Mullins effect, what material should I choose?

Response: We have made changes in our manuscript to address the question. Changes that we made are already indicated in the answer to the Question 4 asked by Reviewer \#1: How one can fight against it?

Comments: p. 4 "In this article, we aim to elucidate one of the most common nonlinear effects experiences by filled elastomers: the Mullins effect". This sentence is misleading. The paper reports data for commercial silicones, with no explanation of the origin of the effect. Nothing is elucidated! In addition, the Mullins effect has already been measured on many elastomers. Finally, the most common nonlinear effect is the non-linear stress-strain curve, not the Mullins effect.

Response: Thank you for your comment. Misleading sentences have been corrected.

Modification in the article on page 4, section -Introduction-: In this work, four commercial silicone formulations (Sylgard 184, Sylgard 186, Ecoflex 00-30 and Ecoflex 00-50), two combinations of commercial silicone formulations (Sylgard 184 and Ecoflex 00-30, Sylgard 184 and Ecoflex 00-50) and two silicone formulations mixed at different stoichiometric ratios (Sylgard 184 and Sylgard 186) were made and tested in order to illustrate commonly encountered strain-induced phenomena. Moreover, correlations between softening effect and different silicone elastomer formulations were made using the strain energy function.

- Question 8: Why did you choose the specific silicones you used? Was there a specific reason?

Response: We have made changes in our manuscript to address the questions.

Modification in the article on page 4, section -Introduction-: Reversible stretchability and flexibility render silicone elastomers suitable materials for soft transducers ${ }^{[32-34]}$, soft robots ${ }^{[35]}$, medical devices ${ }^{[2,36]}$, and many other futuristic applications. Silicone elastomers used for different applications are expected to maintain reliable properties and performance under longterm employment. Accounting for the Mullins effect in silicone formulations offers a simple means of avoiding experimental discrepancies and product unreliability. The Mullins effect has been widely characterized and modeled in natural rubbers with and without fillers dispersed..$^{[7,23,28,31,33,35,37-40]}$ Amongst silicone elastomers, Sylgard formulations are by far the most investigated ones, and Sylgard 184 is often considered as a benchmark for commercial silicone elastomers. ${ }^{[33,35,39]}$ However, the Ecoflex formulations with Shore hardness values ranging from 00-10 to 00-50 have lately gained significant attention in different applications due to their increased softness and stretchability compared to the more rigid Sylgard 184. ${ }^{[34,35,38,41,42]}$ While investigations of Sylgard formulations are extensive, investigations of Ecoflex are still quite limited, and a thorough, consistent characterization of the Ecoflex remains lacking. 